University of Tennessee Health Science Center UTHSC Digital Commons

$12-2016$

\title{
Role of Occludin in the Regulation of Epithelial Tight Junctions
}

Bhargavi Manda

University of Tennessee Health Science Center

Follow this and additional works at: https://dc.uthsc.edu/dissertations

Part of the Medical Cell Biology Commons, and the Medical Physiology Commons

\section{Recommended Citation}

Manda, Bhargavi (http://orcid.org/0000-0001-5618-3967), "Role of Occludin in the Regulation of Epithelial Tight Junctions" (2016). Theses and Dissertations (ETD). Paper 410. http://dx.doi.org/10.21007/ etd.cghs.2016.0415.

This Dissertation is brought to you for free and open access by the College of Graduate Health Sciences at UTHSC Digital Commons. It has been accepted for inclusion in Theses and Dissertations (ETD) by an authorized administrator of UTHSC Digital Commons. For more information, please contact jwelch30@uthsc.edu. 


\title{
Role of Occludin in the Regulation of Epithelial Tight Junctions
}

\begin{abstract}
Tight junctions (TJ) constitute the primary component of epithelial barrier function, a disruption of which is involved in the pathogenesis of many gastrointestinal, pulmonary and renal diseases. Occludin is the major transmembrane protein of TJ, a deletion of which leads to a complex phenotype including chronic inflammation in several epithelial tissues of occludin deficient mice and poor TJ integrity in epithelial cell lines. Its down regulation was seen in Crohn's disease, tumors of the colon, brain, endometrium and breast cancer. Occludin is also known to be a target that enables Hepatitis $C$ Virus infection and bacterial pathogenesis. But the specific function of occludin in TJ remains unknown. Numerous studies implicated that occludin phosphorylation plays a crucial role in TJ regulation. Based on our previous studies, we have identified a conserved phosphorylation hotspot in the C-terminal domain of occludin, called occludin regulatory motif (ORM), which has a potential to confer dynamic properties to occludin. The present study was designed to determine the function of ORM in TJ regulation. Stable, occludin-deficient MDCK cell line (OD-MDCK) that expresses wild type occludin (OcIWT) or a deletion mutant occludin (OcIDM) that lacked ORM were developed. Confocal imaging and immunoprecipitation analyses showed that absence of ORM does not prevent occludin localization and interaction with ZO-1 at TJ. However, FRAP analysis showed that mobile fraction of occludin is reduced by the absence of ORM, suggesting the role of ORM in occludin dynamics. Also, $\mathrm{Ca} 2+$ depletion-induced disruption of TJ, adherens junctions and cytoskeleton and barrier dysfunction were attenuated in the absence of ORM. Mutation anlaysis of ORM phosphosites Y 398, Y 402, T403 and T404, revealed that ORM-mediated phosphorylation events determine the dynamic properties of TJ. Then the role of ORM-mediated protein interactions in TJ modulation was investigated. A synthetic peptide analogous to occludin regulatory region (ORP), which enhanced TJ assembly and attenuated radiation-induced barrier dysfunction in Caco-2 cells, was used as bait to pull down proteins that interact with ORM. Proteomic analyses and pull down assays using ORP and GST tagged occludin Cterminus identified that MAP7 (an epithelial specific microtubule associated protein) interacts with ORM in a tyrosine phosphorylation-dependent mechanism. Furthermore, MAP7 binding to occludin in Caco-2 cells was elevated by treatment with osmotic stress or dextran sodium sulfate, both known to increase occludin Tyr-phosphorylation. These data demonstrated that MAP7 interacts with ORM and mediates occludin redistribution during TJ disruption. In conclusion ORM confers dynamic properties to TJ via its phosphorylation dependent protein-protein interactions. The findings in my project establish the functional significance of ORM in TJ dynamics and regulatory mechanisms associated with $\mathrm{TJ}$ modulation. Identification of proteins that interact with ORM provides new therapeutic targets in the treatment of diseases associated with occludin depletion and epithelial barrier dysfunction.
\end{abstract}

\section{Document Type}

Dissertation

Degree Name

Doctor of Philosophy (PhD)

Program

Biomedical Sciences

Research Advisor

Radhakrishna Rao, Ph.D.

Keywords

Epithelium, Hot Spot, Occludin, Phophorylation, Regulation, Tight Junctions 


\section{Subject Categories}

Medical Cell Biology | Medical Physiology | Medical Sciences | Medicine and Health Sciences

\section{Comments}

Two year embargo expires October 2018. 


\title{
Role of Occludin in the Regulation of Epithelial Tight Junctions
}

\author{
A Dissertation \\ Presented for \\ The Graduate Studies Council \\ The University of Tennessee \\ Health Science Center \\ In Partial Fulfillment \\ Of the Requirements for the Degree \\ Doctor of Philosophy \\ From The University of Tennessee
}

By

Bhargavi Manda

December 2016 
Copyright (C) 2016 by Bhargavi Manda. All rights reserved. 


\section{DEDICATION}

This work is dedicated to my parents who have encouraged me through out my life to pursue a career in science and inspired me to make significant contribution to the research that holds great promise for the betterment of human health. 


\section{ACKNOWLEDGEMENTS}

I would like to acknowledge the people who have guided me through out my entire project. Firstly my principal investigator Dr. Radhakrishna Rao, who has been a great mentor and helped me tremendously in building a promising career path. My committee members Dr. Ramareddy V. Guntaka, David R. Nelson, Michael R. Taylor, Christopher M. Waters, who have directed me in achieving my goals through their valuable suggestions and criticism during every graduate committee presentation.

Dr. Leonard Johnson has given me valuable advice regarding research methodologies during the advanced physiology course work. Dr. Hina Mir generated occludin-deficient MDCK cell line. Dr. Ruchika Gangwar helped me to standardize protocols for ORP pull down studies. Dr. Kamaljith Kaur, Dr. Pradeep K Shukla, Dr. Avtar S Meena, Dr. Mythili K Padala helped me learn various laboratory techniques. Dr. Sandeep Pallikuth, has helped me perform imaging experiments using the confocal microscope. Department of physiology at UTHSC has provided me with the resources and an encouraging work environment. Dr. Charles W. Leffler, Dr. Adebowale Adebiyi and Dr. Kafait U.Malik have provided me with consultant letters for a pre-doctoral fellowship application to AHA.

I am thankful to my parents for putting my education before everything else and encouraging me at every level to pursue science as a career. I am grateful to my in-laws for supporting me right from the beginning of my Ph.D. and taking pride in every small achievement of mine. I am thankful to my husband, Vamshi for being my pillar of strength and my daughter, Anika for being the source of my positive energy. I am thankful to my friends who have given me the moral support through out my Ph.D.

I have been supported by NIH grant-DK55532 to Dr. RK Rao. I was also supported by J. Paul Quigley Memorial scholarship (2013-2014) and Dorothy K. and Daniel L. Gerwin memorial Scholarship (2014-2015). 


\begin{abstract}
Tight junctions (TJ) constitute the primary component of epithelial barrier function, a disruption of which is involved in the pathogenesis of many gastrointestinal, pulmonary and renal diseases. Occludin is the major transmembrane protein of TJ, a deletion of which leads to a complex phenotype including chronic inflammation in several epithelial tissues of occludin deficient mice and poor TJ integrity in epithelial cell lines. Its down regulation was seen in Crohn's disease, tumors of the colon, brain, endometrium and breast cancer. Occludin is also known to be a target that enables Hepatitis C Virus infection and bacterial pathogenesis. But the specific function of occludin in TJ remains unknown. Numerous studies implicated that occludin phosphorylation plays a crucial role in TJ regulation. Based on our previous studies, we have identified a conserved phosphorylation hotspot in the C-terminal domain of occludin, called occludin regulatory motif (ORM), which has a potential to confer dynamic properties to occludin. The present study was designed to determine the function of ORM in TJ regulation.

Stable, occludin-deficient MDCK cell line (OD-MDCK) that expresses wild type occludin $\left(\mathrm{Ocl}^{\mathrm{WT}}\right)$ or a deletion mutant occludin $\left(\mathrm{Ocl}^{\mathrm{DM}}\right)$ that lacked ORM were developed. Confocal imaging and immunoprecipitation analyses showed that absence of ORM does not prevent occludin localization and interaction with ZO-1 at TJ. However, FRAP analysis showed that mobile fraction of occludin is reduced by the absence of ORM, suggesting the role of ORM in occludin dynamics. Also, $\mathrm{Ca}^{2+}$ depletion-induced disruption of TJ, adherens junctions and cytoskeleton and barrier dysfunction were attenuated in the absence of ORM. Mutation anlaysis of ORM phosphosites Y 398, Y 402, T403 and T404, revealed that ORM-mediated phosphorylation events determine the dynamic properties of TJ. Then the role of ORM-mediated protein interactions in TJ modulation was investigated. A synthetic peptide analogous to occludin regulatory region (ORP), which enhanced TJ assembly and attenuated radiation-induced barrier dysfunction in Caco-2 cells, was used as bait to pull down proteins that interact with ORM. Proteomic analyses and pull down assays using ORP and GST tagged occludin Cterminus identified that MAP7 (an epithelial specific microtubule associated protein) interacts with ORM in a tyrosine phosphorylation-dependent mechanism. Furthermore, MAP7 binding to occludin in Caco-2 cells was elevated by treatment with osmotic stress or dextran sodium sulfate, both known to increase occludin Tyr-phosphorylation. These data demonstrated that MAP7 interacts with ORM and mediates occludin redistribution during TJ disruption.
\end{abstract}

In conclusion, ORM confers dynamic properties to $\mathrm{TJ}$ via its phosphorylation dependent protein-protein interactions. The findings in my project establish the functional significance of ORM in TJ dynamics and regulatory mechanisms associated with TJ modulation. Identification of proteins that interact with ORM provides new therapeutic targets in the treatment of diseases associated with occludin depletion and epithelial barrier dysfunction. 


\section{TABLE OF CONTENTS}

\section{CHAPTER 1. INTRODUCTION ..................................................................................}

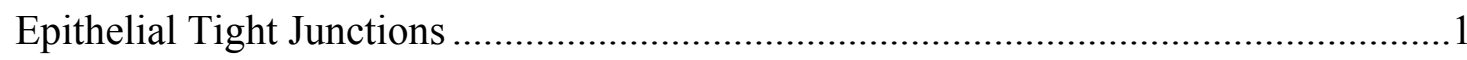

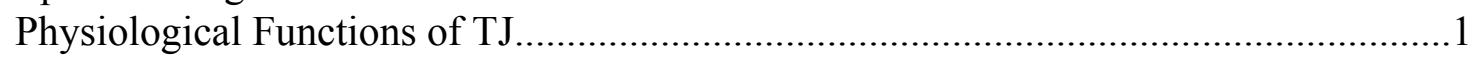

Early Discovery of TJ Components....................................................................



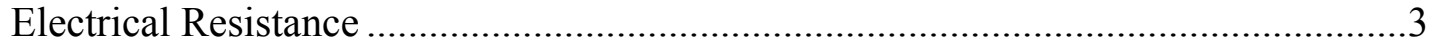

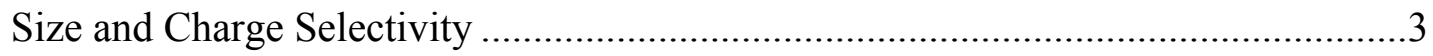

Pore and Leak Pathways ....................................................................................4

Measurement of Electrical Resistance, Size and Charge Selectivity.........................4

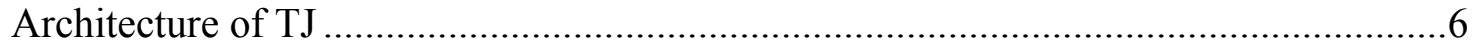

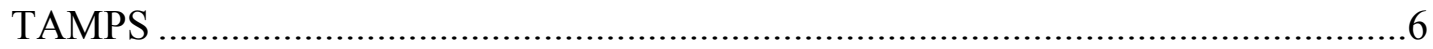

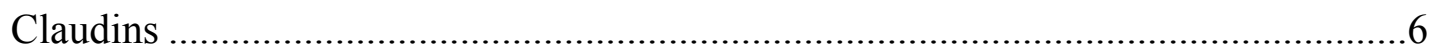

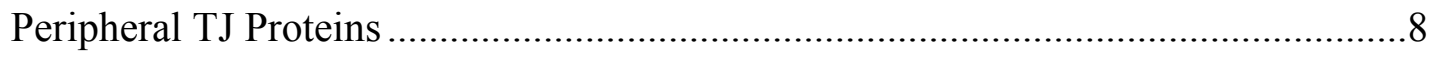

Occludin Structure ..........................................................................................

Occludin C-terminus Harbors a Conserved Phosphorylation Hot Spot ......................8

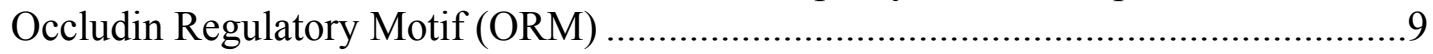

Occludin Function............................................................................................... 9

TJ- a Dynamic Multi-Protein Complex at Steady State ...........................................11

Occludin Exchange Occurs by Diffusion within and Between TJ and Lateral



ZO-1 Exchanges with an Intracellular Pool ................................................... 13

Claudin-1 Exchange Occurs by Diffusion within the Membrane.............................13

TJ Regulation by Occludin Phosphorylation ...........................................................13

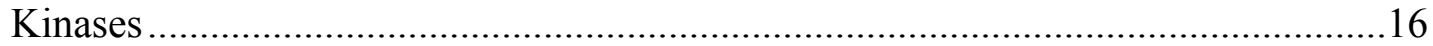

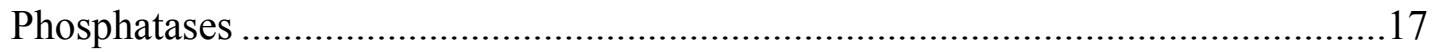

Cytokine-induced TJ Regulation Involves Occludin .........................................18

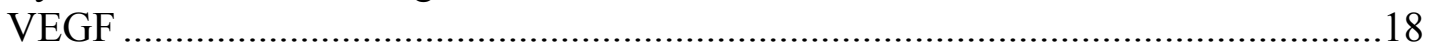

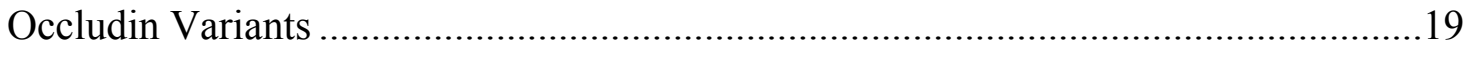



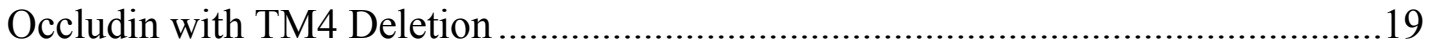

Occludin with Alternate Promoter (Exon 1a) .......................................................19

Occludin with Alternate Promoter and Exon 9 Deletion ......................................19

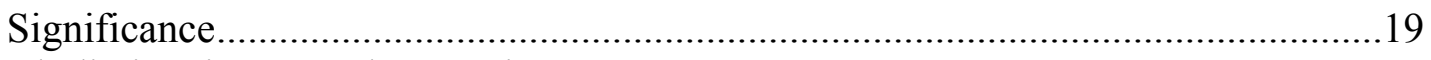

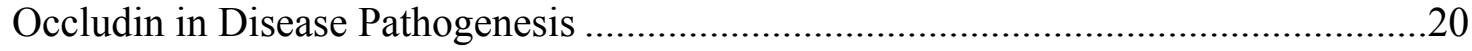

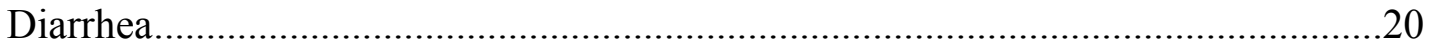

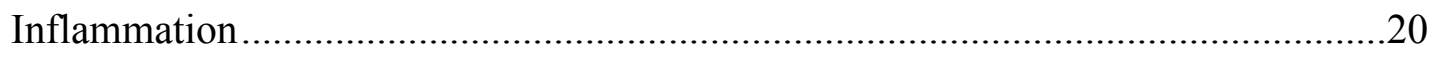

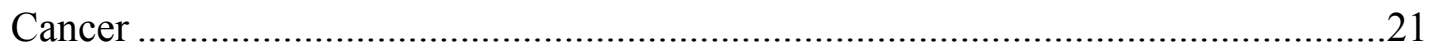

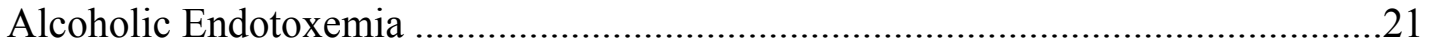

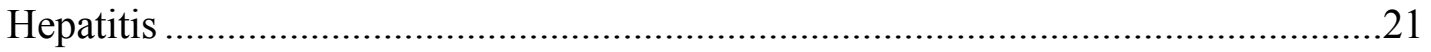

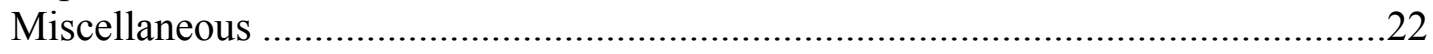

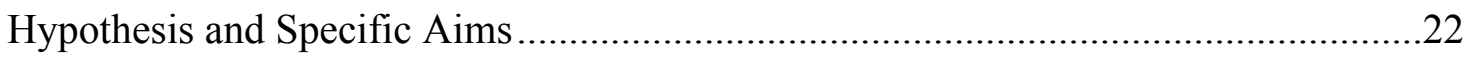

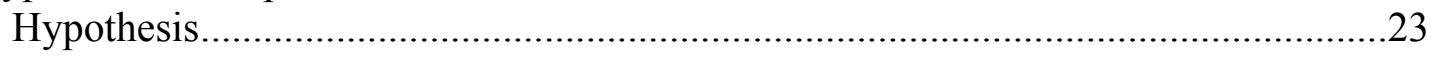


Specific Aim 1: To Determine That ORM Confers Dynamics to TJ in a Phosphorylation-dependent Mechanism

Specific Aim 2: To Determine That ORM-mediated Protein Interactions Are

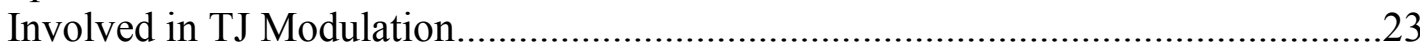

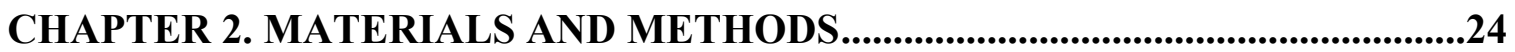

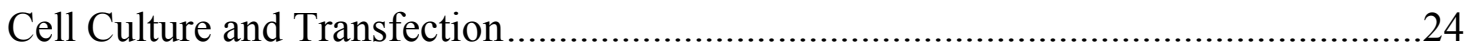

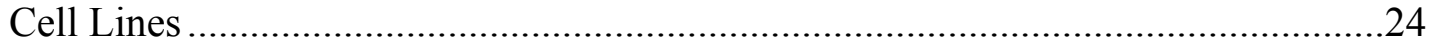

Occludin Gene Silencing .............................................................................2



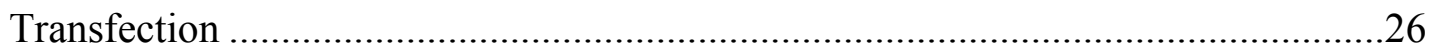

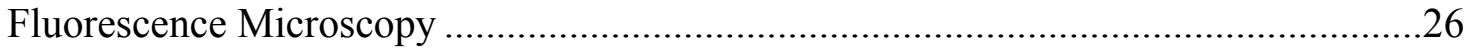



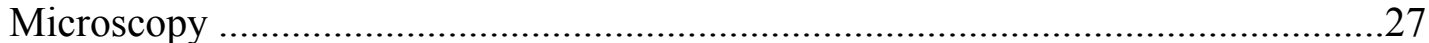

Analysis of Barrier Function and TJ Integrity ..........................................................27

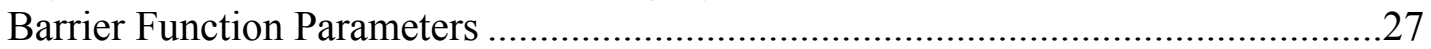

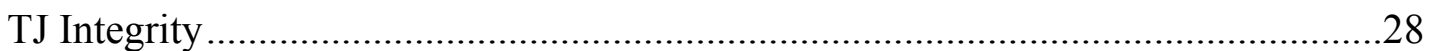

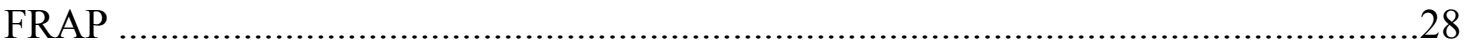

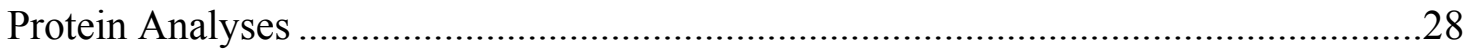

Preparation of Triton-soluble and Triton-insoluble Fractions ...................................28

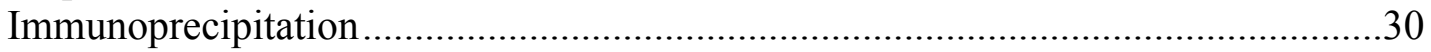

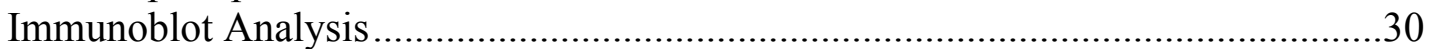

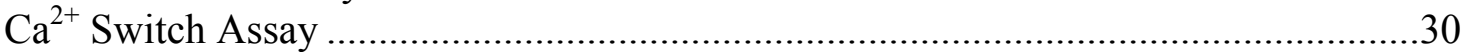

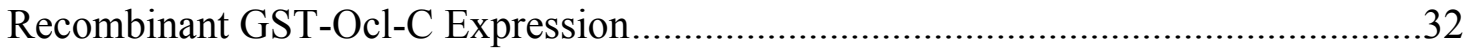

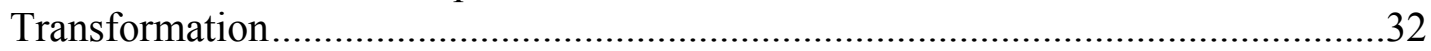



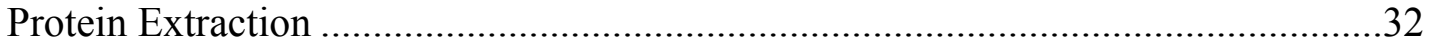

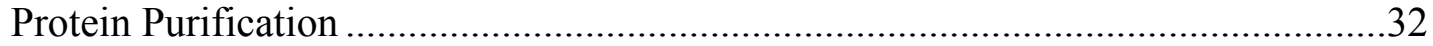

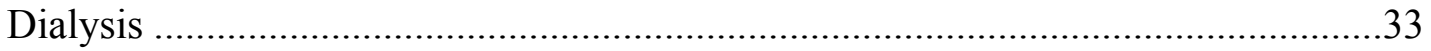

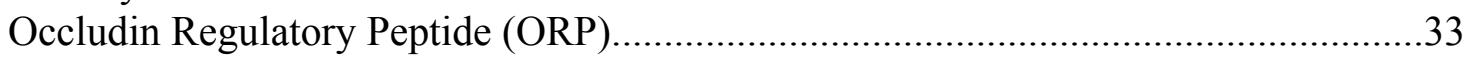

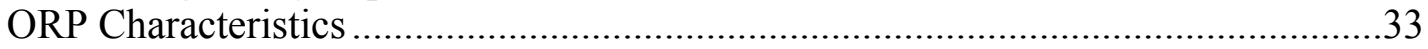

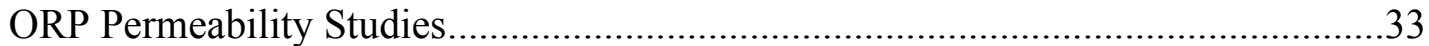

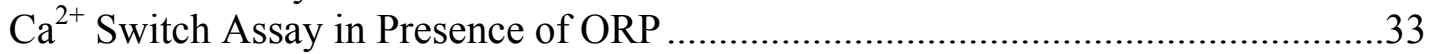

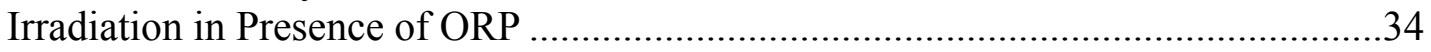

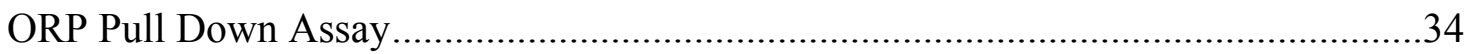

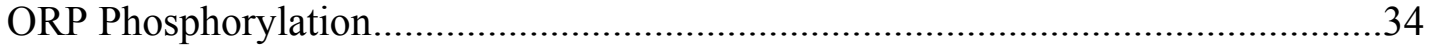

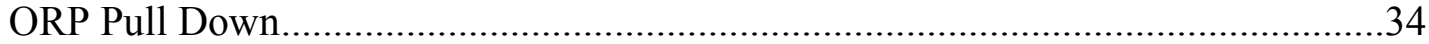

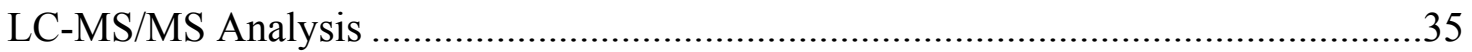

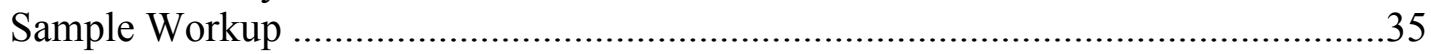

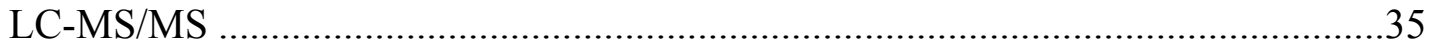

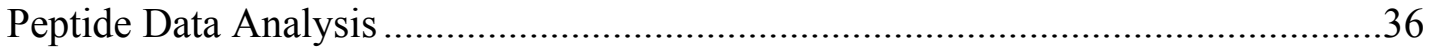

Protein Differential Expression Analysis...............................................................36



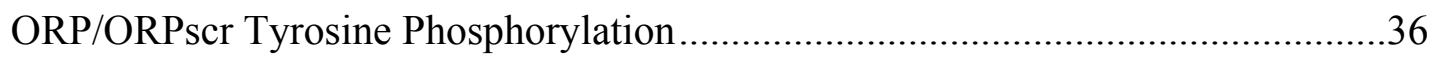

GST-Ocl-C/GST Tyrosine Phosphorylation ............................................................36

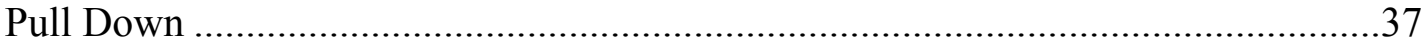




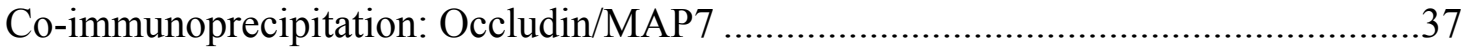

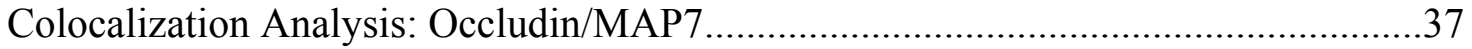

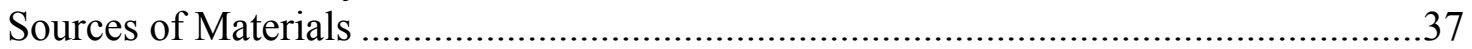

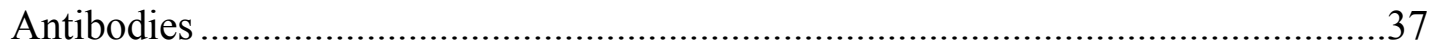

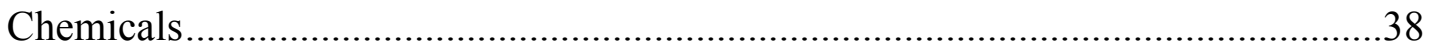

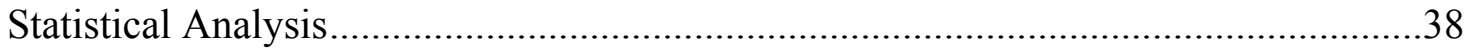

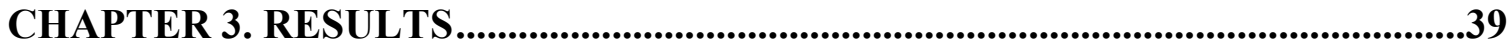

Specific Aim 1: To Determine That ORM Confers Dynamics to TJ in a

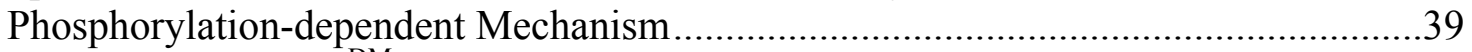



Deletion of ORM Does Not Prevent Occludin Assembly at the TJ ..........................39

Absence of ORM in Occludin Does Not Prevent Its Interaction with ZO-1 ..............39

Lack of Both ORM and Occludin Decreases Steady-state Resistance but Does

Not Affect Leak Pathway Permeability ....................................................................42

Lack of ORM or Occludin Enhances Claudin-2 Expression at the TJ ......................42

Lack of Both ORM and Occludin Enhances Cytoskeletal Organization and

Occludin Localization at TJ ..............................................................................45

Deletion of ORM Reduces Occludin Mobility at the Intercellular Junctions.............45

ORM Deletion Confers Resistance to $\mathrm{Ca}^{2+}$ Depletion-mediated Occludin

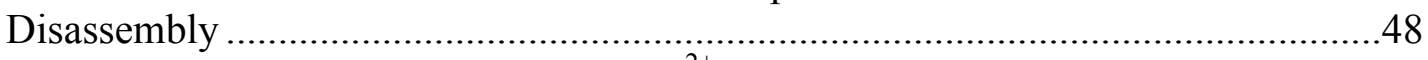

ORM Deletion Confers Resistance to $\mathrm{Ca}^{2+}$ Depletion-mediated Barrier

Dysfunction .................................................................................................... 48

Deletion of ORM Confers Resistance to Disruption of Apical Junctional

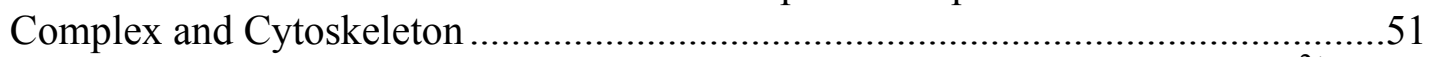

ORM Deletion Attenuates TJ and AJ Disruption Induced by EGTA-based $\mathrm{Ca}^{2+}$

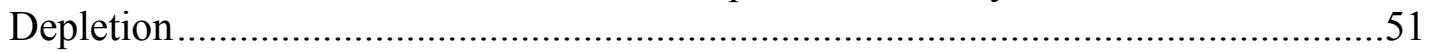

Deletion of ORM Does Not Prevent CytochalasinD-mediated Disruption of TJ

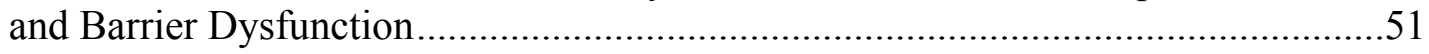

Deletion of ORM Does Not Prevent Osmotic Stress-mediated Disruption of TJ

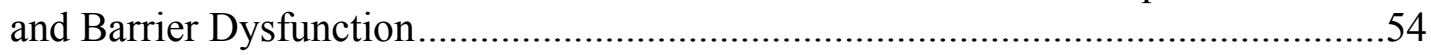

Phoshorylation of ORM on T403/403 and Y398/402 Determines Dynamic

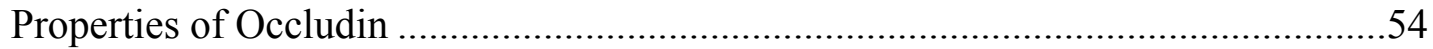

Phosphorylation of ORM on T403/403 and Y398/402 Determines Dynamic

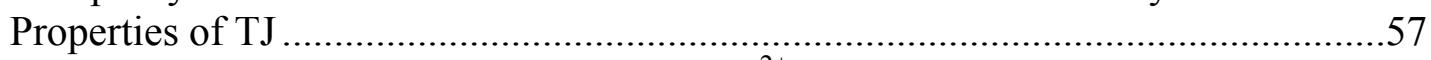

Tyrosine Phosphorylation Plays a Role in $\mathrm{Ca}^{2+}$ Depletion-mediated TJ

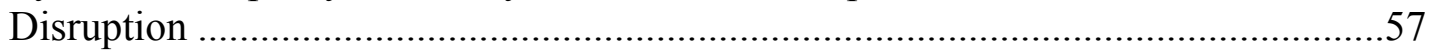

Specific Aim 2: To Determine That ORM-mediated Protein Interactions Are

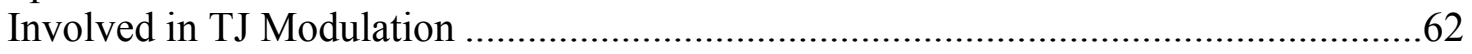

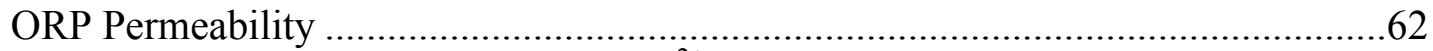

ORP Enhances TJ Assembly During $\mathrm{Ca}^{2+}$ Switch Assay in Caco-2 Cells .................62

ORP Attenuates Radiation-induced Barrier Dysfunction and TJ Disruption .............65

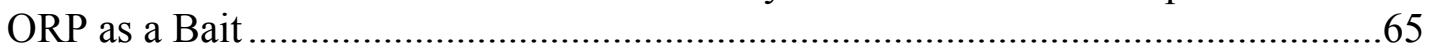

MAP7 Binds to Tyrosine-Phosphorylated ORP and GST-Ocl-C ..............................69

DSS or Osmotic Stress Enhances Interaction Between Occludin and MAP7 ...........69

MAP7 Colocalizes with Occludin in Irradiated Mouse Colon ....................................73 
CHAPTER 4. DISCUSSION

Specific Aim 1: To Determine That ORM Confers Dynamics to TJ in a

Phosphorylation-dependent Mechanism . .76

ORM Is Not Required for Assembly of Occludin into TJ ....................................76

ORM Deletion Does Not Affect Barrier Development by Leak Pathway Flux ........77

ORM Deletion Enhances Occludin Localization and Reduces Its Mobility at the



ORM Deletion Specifically Attenuates $\mathrm{Ca}^{2+}$ Depletion-mediated Disruption of

Apical Junctional Complexes and Barrier Dysfunction...........................................78

Phosphorylation of ORM on T403/T404 and Y398/Y402 Determines the

Dynamic Properties of TJ

Specific Aim 2: To Determine That ORM-mediated Protein Interactions Are

Involved in TJ Modulation

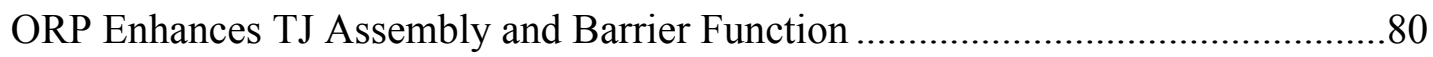

ORP Interacts with Proteins in a Phosphorylation-dependent Manner .....................80

MAP7 Interacts with Occludin by a Phosphorylation-dependent Mechanism ..........81

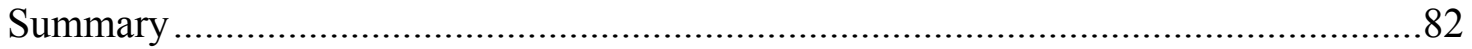



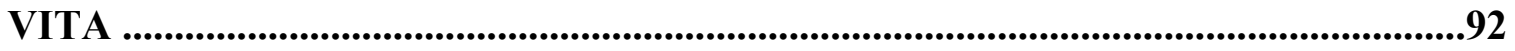




\section{LIST OF TABLES}

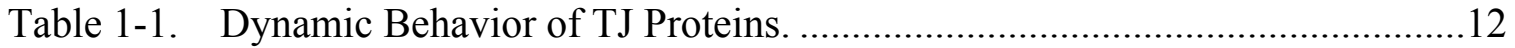

Table 1-2. Kinases and Phosphatases Associated with Occludin Phosphorylation........14

Table 1-3. Mutation Analyses of Phosphorylation Hotspot in Occludin.......................15

Table 2-1. Primers for Knockdown and Mutation of Occludin....................................25

Table 3-1. ORM-interacting Proteins Identified by LC-MS/MS...............................70 


\section{LIST OF FIGURES}

Figure 1-1. Intercellular Apical Junctional Complex and Tight Junctions.....................2

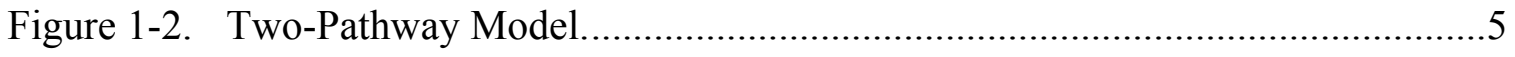

Figure 1-3. Tight Junction- a Multi-protein Complex. ...............................................

Figure 1-4. Conserved-phosphorylation Hot Spot in Occudin C-terminus. ...................10

Figure 2-1. Fluorescence Recovery after Photobleaching (FRAP). ............................29

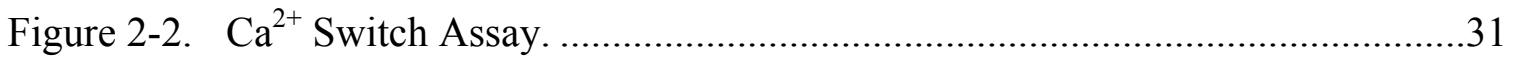

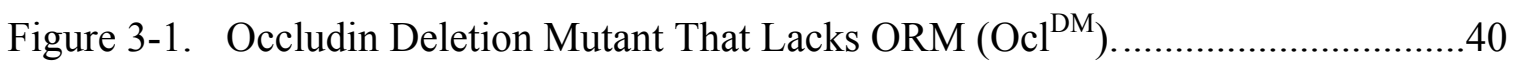

Figure 3-2. ORM Deletion Does Not Prevent Assembly of Occludin into TJ...............41

Figure 3-3. ORM Deletion in Occludin Does Not Prevent Interaction with ZO-1........43

Figure 3-4. ORM Deletion Decreases Steady-state Resistance of the Barrier. ..............43

Figure 3-5. Absence of Occludin or ORM Enhances Claudin-2 Localization at TJ.....44

Figure 3-6. Deletion of ORM Enhances Association of Occludin with Actin-rich



Figure 3-7. ORM Deletion Reduced Mobile Fraction of Occludin.............................47

Figure 3-8. ORM Deletion Attenuates $\mathrm{Ca}^{2+}$ Depletion-mediated Occudin Disassembly.

Figure 3-9. ORM Deletion Attenuates $\mathrm{Ca}^{2+}$ Depletion-induced Barrier Dysfunction. ...50

Figure 3-10. ORM Deletion Attenuates $\mathrm{Ca}^{2+}$ Depletion-mediated Disruption of Apical Junctional Complex and Cytoskeleton...................................................52

Figure 3-11. ORM Deletion Attenuates EGTA-mediated TJ and AJ Disruption. 53

Figure 3-12. ORM Deletion Does Not Prevent Cytochalasin-mediated TJ Disruption and Barrier Dysfunction.

Figure 3-13. ORM Deletion Does Not Prevent Osmotic Stress-mediated TJ

Disruption and Barrier Dysfunction. .56

Figure 3-14. ORM Confers Dynamic Properties to Occludin in a Phosphorylationdependent Manner. 
Figure 3-15. ORM Phosphorylation Determines Mobility of Occludin at TJ.

Figure 3-16. ORM Determines Barrier Function in a Phosphorylation-dependent

Mechanism.

Figure 3-17. ORM Confers Dynamic Properties to TJ in a Phosphorylation-dependent Mechanism.

Figure 3-18. $\mathrm{Ca}^{2+}$ Depletion-induced TJ Disruption Involves Tyrosine Phosphorylation.

Figure 3-19. ORP Sequence and Its Permeability ....................................................64

Figure 3-20. ORP Enhances TJ Assembly. ..........................................................66

Figure 3-21. ORP Attenuates Radiation-induced Barrier Dysfunction..........................67

Figure 3-22. ORP Attenuates Radiation-induced TJ Disruption. .................................68

Figure 3-23. Ion Spectra Profiles of Peptides Corresponding to the Proteins Identified

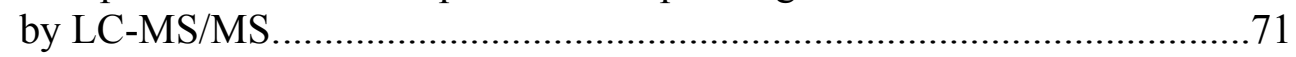

Figure 3-24. Immunoblot Analyses for Proteins Pulled Down by ORP.........................72

Figure 3-25. MAP7 Interacts with Tyrosine-phosphorylated ORP and Occludin Cterminus.

Figure 3-26. MAP7 Interacts with Occludin in a Tyrosine phosphorylation-dependent Mechanism.

Figure 3-27. MAP7 Colocalizes with Occludin in Radiation Exposed Mouse Colon. .....75

Figure 4-1. MAP7 Mediates Trafficking of Occludin During TJ Regulation. .83 


\section{LIST OF ABBREVIATIONS}

AJ

APC

DS

EL

FLIP

FRAP

GFP

GSH

GST

$\mathrm{HCV}$

HSPD1

IBD

IL

JAM

LCM

MAP7

MDCK

MLCK

NCM

OD

ORM

PKP3

PP2A

PRDX1

RhoK

ROI

SGLT-1

TAMP

TER

TJ

TNF

VEGF

ZO-1
Adherens junction

Apical junctional complex

Desmosome

Extracellular loop

Fluorescence loss in photo bleaching

Fluorescence recovery after photobleaching

Green fluorescent protein

Glutathione

Glutathione S tranferase

Hepatitis C virus

Heat shock protein family D-1

Inflammatory bowel disease

Intracellular loop

Junction adhesion molecule

Low calcium media

Microtubule associated protein 7

Madin-Darby canine Kidney

Myosin light chain kinase

Normal calcium media

Occludin deficient

Occludin regulatory motif

Plakophilin 3

Protein phosphatase 2A

Peroxiredoxin 1

Rho Kinase

Region of interest

Sodium-glucose linked transporter-1

Transmembrane associated MARVEL protein

Transepithelial electrical resistance

Tight junction

Tumor necrosis factor

Vascular endothelial growth factor

Zonula occludens-1 


\section{CHAPTER 1. INTRODUCTION}

\section{Epithelial Tight Junctions}

Luminal and vascular fluid spaces are delimited by epithelial and endothelial barriers. The characteristic property of forming a barrier between external and internal milieu that generates and maintains physiological functioning of compartments is mainly attributed to the intercellular junctions. These include tight junctions (TJ; also Zonula occludens), adherens junctions (Zonula adhaerens), desmosomes (macula adhaerens) and gap junctions (Figure 1-1A).

Tight junctions (TJ), originally identified by Farquhar and Palade in the early 1960s, are the most apical sites of cell-cell contact (1). On a freeze-fracture micrograph, TJ appear as a belt-like ring of continuous anastomosing strands sealing and eliminating the intercellular space (Figure 1-1B) (2). This led to the early belief that TJ form an inert and impervious barrier, which later was disproved as TJ components have now been identified to be under continuous remodeling at steady state (3). TJ do not seal the paracellular space completely but regulate the paracellular transport of solutes based on charge and size. Modulation of barrier properties of TJ in response to physiological, pharmacological and pathological stimuli has been demonstrated in several studies (4-9).

\section{Physiological Functions of TJ}

Epithelial TJ serve two main functions: a "gate function" where the TJ strands from neighboring plasma membranes associate with each other and provide selective permeability to ions, water and solutes through paracellular diffusion and a "fence function" that prevents intramembrane diffusion of proteins and thus confers polarity to cells $(10,11)$. Besides their role in these classical functions, several studies reported that TJ play an important role in signal transduction (12-14).

\section{Early Discovery of TJ Components}

The first component of TJ was discovered in 1986 in mammalian epithelial and endothelial cells and named as Zonula occludens-1 (ZO-1; $225 \mathrm{kDa})$ (15). The next TJ associated protein called Cingulin $(160 \mathrm{kD})$ was discovered in in 1988, in avian brushborder cells (16). Both of these are not integral membrane proteins and the search for membrane associated TJ components continued. Occludin $(65 \mathrm{kD})$ was the first integral membrane protein of the TJ to be discovered by Furuse et al in 1993 (17). Occludin is a tetra spanning membrane component of the TJ strands, which interacts with ZO-1 and confers adhesive properties to cells $(18,19)$. Then another class of transmembrane proteins with no sequence similarity and phylogenetically unrelated to occludin called claudins (21-34 kD) were discovered in 1988 (20). Claudin family of proteins includes nearly 27 members, mutations of which cause hereditary diseases (21). 




Figure 1-1. Intercellular Apical Junctional Complex and Tight Junctions.

A. Schematic shows intercellular junctions between intestinal epithelial cells. B. Electron micrograph of mouse intestinal epithelial cells shows intercellular junctions. Inset shows freeze fracture micrograph of tight junctions as continuous anastamosing strands present at the apical end. Scale bar is 200nm. (Mv, microvilli; TJ, tight junction; AJ, adherens junction; DS, Desmosome; Ap, apical membrane; Bl, basolateral membrane).

Source: Reprinted by permission from Macmillan Publishers Ltd: Tsukita, S., Furuse, M., and Itoh, M. (2001) Multifunctional strands in tight junctions. Nat Rev Mol Cell Biol 2, 285-293. Copyright C 2001. 


\section{Characteristics of $\mathbf{T J}$}

\section{Electrical Resistance}

The two-membrane model suggested that apical and basal membranes differ in their conductance properties. Sodium passes across the apical membrane via the $\mathrm{Na}$ channels into the cells down its concentration gradient, while it is pumped across the basolateral membrane via energy dependent Na,K-ATPase. The shunt pathway suggested that this electrical gradient generated by the active transport of $\mathrm{Na}+$ leads to passive diffusion of $\mathrm{Cl}$ - ions through the paracellular route. This revealed that the transcellular and paracellular transport are physiologically coupled.

Transporters in the apical and basolateral membranes mediate transcellular transport. According to the electrical circuit model, the series elements of the resistance of apical $\left(\mathrm{R}_{\text {apical }}\right)$ and basolateral $\left(\mathrm{R}_{\text {basolateral }}\right)$ membranes are in parallel to the resistance of the TJ $\left(R_{T J}\right)$. Because the resistance of cell membranes $\left(R_{\text {apical }}\right.$ and $\left.R_{\text {basolateral }}\right)$ is usually high, it is the resistance of $\mathrm{TJ}\left(\mathrm{R}_{\mathrm{TJ}}\right)$ that determines the transepithelial electrical resistance (TER). It is well accepted that the resistance differs significantly among various tissues and hence referred to as either "tight" or "leaky" epithelia. A good example of a leaky epithelium is that of the mammalian proximal tubule with TER $\sim 6 \Omega . \mathrm{cm}^{2}$, and an example of a tight epithelium is that of a toad urinary bladder with TER $\sim 300,000 \Omega . \mathrm{cm}^{2}$. The physiological significance of the differences in TER becomes evident when we look at the specific function of the epithelium. In the distal nephron, the "tight" TJ are responsible for maintaining high electrochemical gradient generated by the active transcellular transport. Except colon all the segments of gastrointestinal tract are leaky, as higher concentration gradients are to be maintained for reabsorption of $\mathrm{NaCl}$ and water and hence exhibit a higher resistance.

In most cases, the tightness of the epithelia correlated with the number of TJ strands, where tight TJ had more than five strands while the leaky TJ had one single strand. Here it was believed that the resistance of each strand was in series with that of the other strands which is basically the sum of resistances of all the strands and hence has a linear relationship. This was not true in the case of two strains of MDCK, which exhibited several fold difference in TER although they had same number of TJ strands.

\section{Size and Charge Selectivity}

Another model suggested that TJ strands were assumed to be pores that can be open or closed. As TER is measured instantaneously, the resistance was believed to be proportional to the open probability of TJ pores raised to the negative power of number of TJ strands. But this could neither explain the paracellular charge selectivity of different epithelia nor the size selectivity within the same epithelium. It was seen that, along the crypt villus axis in rat jejunum, there was an apparent difference in size selectivity. The upper villus portion allows solutes of radii up to $\sim 6 \mathrm{~A}^{0}$, the lower villus allows solutes of radii up to $\sim 10 \mathrm{~A}^{0}$ while the crypt allows much larger solutes with radii up to $\sim 60 \mathrm{~A}^{0}$. 
Caco-2 cell line known to exhibit villous differentiation has been shown to have 3 distinct pathways similar to that of rat jejunum. These include one that allows passage of

molecules with radii less than $4 \mathrm{~A}^{0}$, one that allows passage of molecules of radii $6.5 \mathrm{~A}^{0}$ and one that allows paracellular transport of much larger non-charged molecules.

\section{Pore and Leak Pathways}

From several studies performed later on to characterize the size selectivity of different epithelia, it was discovered that the paracellular route of transport in itself has two distinct pathways referred to as pore and leak pathways. These two pathways together contribute to the gate function on the TJ.

According to the two pathway model (21) (Figure 1-2), pore pathway is a high capacity, charge selective pathway that allows passage of small ions and solutes less than $\sim 4 \mathrm{~A}^{0}$ in size. Since this pathway is capable of ionic charge discrimination, it is responsible for carrying most of the electrical current during transport. It is now known that a subset of claudins, have the capability to form pores that confer size and charge selectivity. It is the composition of claudins that determines the charge selectivity of the epithelial barrier.

Leak pathway is a low and non-charge selective pathway that allows passage of larger solutes. Solutes larger than $\sim 4 \mathrm{~A}^{0}$ in size are transported through the leak pathway. Flux studies using tracers like mannitol $\left(4.2 \mathrm{~A}^{0}\right)$ and inulin $\left(15 \mathrm{~A}^{0}\right)$ represents permeability through the leak pathway. It has been observed that the proinflammatory cytokines can increase flux through the leak pathway without altering the pore pathway. Regulation of this non-pore pathway is known to involve myosin light chain kinase activity and dynamics of cytoskeleton.

\section{Measurement of Electrical Resistance, Size and Charge Selectivity}

TER is a measure of the flux of all ions across the epithelium which is best represented in leaky epithelia where ion conductance across $\mathrm{TJ}$ is far greater than that of apical and basolateral membranes. $\mathrm{Na}^{+}$and $\mathrm{Cl}^{-}$are the most common ions present in the physiological solutions and they do not discriminate between pore and leak pathways. Any change in either of the pathways reflects in TER. Hence TER cannot be used to study size and charge selectivity of the epithelium.

Charge selectivity can be studied by dilution potential technique. Creating an electrochemical gradient by isosmotic alteration of $\mathrm{NaCl}$ concentration across the epithelium provides a new equilibrium potential now based on the relative permeability of $\mathrm{Na}^{+}$and $\mathrm{Cl}^{-}$ions. Permeability ratio ranges from 10 to 0.1 in different epithelia. 




Figure 1-2. Two-Pathway Model.

Permeability of non-charged polyethylene glycol molecules of various sizes across an epithelium is plotted against their radius. The pathway for molecules smaller than $4 \mathrm{~A}^{0}$ in diameter, referred to as pore pathway, is dictated by size and charge selective claudins. The pathway for molecules larger than $4 \mathrm{~A}^{0}$ in diameter, referred to as a leak pathway, is not charge selective and is governed by the status of cell-cell adhesion and cytoskeleton. Source: Reprinted with permission. Anderson, J. M., and Van Itallie, C. M. (2009) Physiology and function of the tight junction. Cold Spring Harb Perspect Biol 1, a002584. Copyright (C) 2009 Cold Spring Harbor Laboratory Press. 
Bi-ionic substitution is a technique used to determine the charge selectivity of the barrier. This involves replacement of $\mathrm{Na}^{+}$on one side of the epithelium with organic cations of various sizes, measuring the permeability of each organic cation, which in turn determines the size selectivity of pore pathway. Permeability through the leak pathway can be determined by measuring flux of large tracer molecules like mannitol, sucrose, and inulin. Polymers of varying sizes such as polyethylene glycols or dextrans are also widely used to determine paracellular permeability.

\section{Architecture of TJ}

The typical architecture of TJ involves integral membrane proteins of three major families: transmembrane associated marvel protein family (TAMP) comprising of occludin, tricellulin and marvel D3 (1), claudin family and immunoglobulin superfamily members such as junction adhesion molecules (JAMs) (8). A perijunctional actomyosin ring comprising of a thick band of actin and myosin II is present just below the TJ. The $\mathrm{C}$-terminal domains of the integral proteins interact with intracellular PDZ-domain containing scaffolding proteins such as ZO-1, ZO-2 and ZO-3, which in turn crosslink membrane proteins with actin cytoskeleton (2-4) (Figure 1-3). TJ protein complex recruits classic-signaling molecules like PKCs, c-Src, c-Yes, PP2A, PP1 and transcription factors like $\mathrm{ZONAB}$, thus forming a regulatory platform for mediating responses to extracellular and intracellular stimuli (14).

\section{TAMPS}

This family of TJ proteins includes occludin, tricellulin (also called MarvelD2) and Marvel D3. These proteins have a conserved MARVEL (MAL and related proteins for vesicle trafficking and membrane link) domain with four transmembrane domains linked by two extracellular (EL) loops and one intracellular loop (IL) (22). Occludin forms homophilic interactions at the bicellular junctions via its extracellular domains. Tricellulin is localized at tricellular junctions and is believed to form a barrier for macro molecules (23). MarvelD3 is mainly localized at the bicellular junctions and is not required for $\mathrm{TJ}$ formation (22).

\section{Claudins}

To date, this TJ protein family includes nearly 27 different claudins. Their domain structure includes an intracellular $\mathrm{N}$-terminus, a longer $\mathrm{C}$-terminus, two extracellular loops and an intracellular loop. The first extracellular loop consists of a signature sequence and a C-terminus PDZ domain-binding motif, which interacts with ZO-1.

All claudins are barrier-forming proteins, however some claudins are considered to be pore-forming based on their ability to increase paracellular permeability specifically to molecules of certain size or charge but do not affect the barrier permeability to 


\section{Lumen}

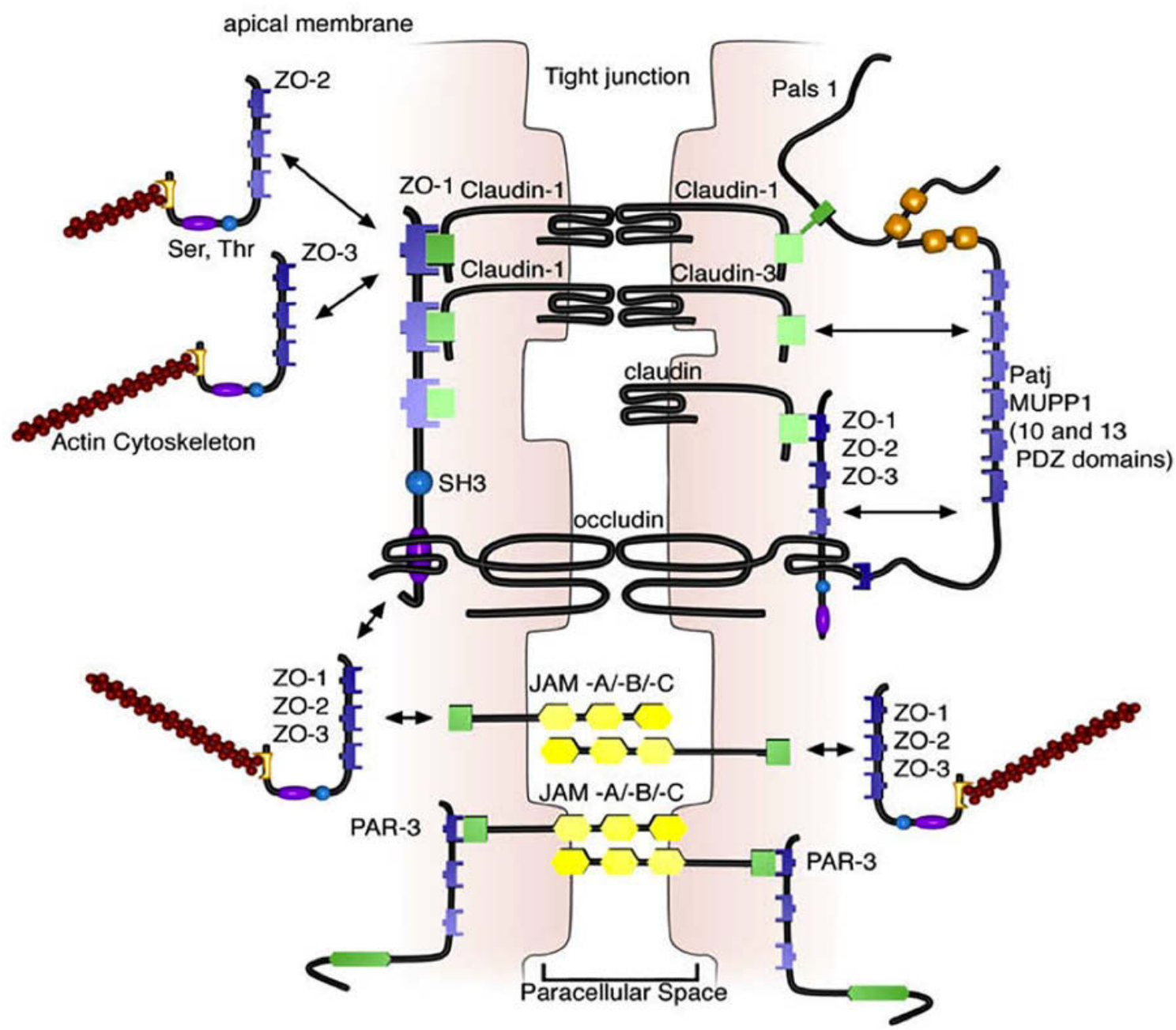

Figure 1-3. Tight Junction- a Multi-protein Complex.

Epithelial tight junctions comprise of integral transmembrane proteins such as occludin, claudins, and junction adhesion molecules (JAM) that seal the paracellular space. The intracellular tails of these membrane proteins interact with scaffolding proteins such as ZO-1, ZO-2, ZO-3 which then cross link membrane proteins with the actin cytoskeleton. Source: Reprinted with permission. Groschwitz, K. R., and Hogan, S. P. (2009) Intestinal barrier function: molecular regulation and disease pathogenesis. J Allergy Clin Immunol 124, 3-20. Copyright (C) 2009 American Academy of Allergy, Asthma \& Immunology. 
macromolecules. Claudin-2, -10b, 15 and 16 are known to be cationic pore forming claudins while claudin-10a and -17 are anionic pore forming claudins. Mutations in these genes are known to cause hereditary diseases (24). Claudin-2 is a well-studied cationic pore forming claudin that is highly expressed in leaky TJ like that of proximal tubule and intestinal crypts. In the proximal tubule, claudin-2 is responsible for passive reabsorption of sodium $(25,26)$.

\section{Peripheral TJ Proteins}

The intracellular scaffolding proteins include zonula occludens proteins (ZO-1, ZO-2, ZO-3), MAGI proteins (MAGI1, MAGI2 and MAGI3), Par-3, Par-6, MUPP and PatJ. The ZO proteins belong to the MAGUK (membrane-associated guanylate kinase homologues) protein family as their domain composition includes 3 PDZ domains in the $\mathrm{N}$-terminus, a SH3 homology domain and a guanylate kinase domain. The guanylate kinase domain of ZO-1 interacts with occludin c-terminus. Cingulin is associated with the cytoplasmic surface of TJ and expressed during early embryogenesis and epithelial maturation. Cingulin links TJ proteins to actin cytoskeleton via interactions with $\mathrm{ZO}$ proteins and actin. ZONAB. Rab13 (27).

\section{Occludin Structure}

Occludin is expressed in various epithelial cells but is not detectable in fibroblasts. Human occludin gene was mapped to chromosome band 5q.13.1. Nearly 90\% homology exists among mammalian occludins (20). The multidomain tetra span structure includes four transmembrane domains (TM), two extracellular loops (EL1 \& EL2), a cytoplasmic short N-terminus and an extended C-terminus. EL1 and EL2 are involved in cis and trans homophilic interactions (28). The coiled coil domain in occludin C-terminus interacts directly with the guanylate kinase-like (GUK) domain of ZO-1 (29). Nearly half of the 522 amino acid residues of occludin (266-522) are present in its C-terminus and is a known target for several kinases and phosphatases (30). Multiple bands of occludin are often detected by immunoblot analysis, which are believed to be phosphorylated forms of occludin. Most of the studies were performed using antibodies that detect occludin band around $65 \mathrm{kDa}$.

\section{Occludin C-terminus Harbors a Conserved Phosphorylation Hot Spot}

Structural analysis of occludin C-terminus (383-522) revealed that the distal part of this domain, comprising of amino acids from 416 to 522, is involved in an ordered coiled-coil structure that interacts with ZO-1 while the region comprising of N-terminus 34 amino acids is disordered (31). This proximal disordered region includes a sequence of amino acids highly conserved among mammalian species. Most notably this includes a phosphorylation hotspot stretching from Y-398 to S-408. 


\section{Occludin Regulatory Motif (ORM)}

Previous studies showed that occludin undergoes tyrosine phosphorylation that regulates its interaction with $\mathrm{ZO}-1$ during $\mathrm{H}_{2} \mathrm{O}_{2}$ induced TJ disruption $(32,33)$. Elias et al highlighted the presence of a highly conserved phosphorylation hot spot (Y398-S408) in occludin C-terminus (Figure 1-4A) and identified that phosphorylation of Y-398 and Y402 attenuates its interaction with ZO-1 (34). Suzuki et al showed that PKC $\eta$ mediated phosphorylation of T403 and T404 is involved in the regulation of epithelial TJ (35). It was believed that tyrosine phosphorylation (Y-398, Y-402) by c-Src, destabilizes TJ whereas threonine phosphorylation (T403, T404) by $\mathrm{PKC} \zeta$ and $\mathrm{PKC} \eta$ promotes TJ assembly (36). Studies by Turner group demonstrated the role of S408 phosphorylation in the regulation of barrier function (37). Later on, Dorfel et al showed that CK2-mediated serine/threonine phosphorylation of the T400-XXX-T404-XXX-S408 motif regulates TJ modulation (38). Although the structure of this phosphorylation hot spot (Y398-S408) was not resolved by crystallography, based on the observations that this region is a target for phosphorylation mediated TJ regulation, we considered this motif a functional component of occludin and coined the term "occludin regulatory motif" (ORM) (Figure 1-4B).

\section{Occludin Function}

Overexpression of occludin in insect cells resulted in occludin localization at the intracellular multi-lamellar bodies similar to TJ (39). Expression of occludin in MDCK cells resulted in occludin localization at intercellular junctions with increased TER and increased paracellular permeability (40). Occludin expression in fibroblasts that are occludin deficient but express ZO-1 and AJ proteins, occludin was localized at cell-cell contacts and conferred cell adhesion (19). These data suggested that occludin is required for formation of TJ. However, alteration of both alleles of occludin in embryonic stem cells did not prevent formation of TJ strands, suggesting that other TJ associated proteins can form TJ strands even in the absence of occludin (41).

Saitou et al generated occludin -/-mice that carried a null mutation in occludin gene, to study its function in vivo. The heterozygous and homozygous mutant mice were viable with no gross phenotype at birth but exhibited post-natal growth retardation. There were no significant changes in the formation of TJ strands (in terms of their number, size and appearance) in mutant mice compared to wild type mice, as visualized by ultrathinsection electron microscopy and freeze fracture replica electron microscopy. TJ were similarly not affected in kidney or liver of the occludin -/- mice. TER of the small and large intestinal epithelial layers was low in mutant mice but not statistically significant. Histological examination revealed chronic inflammation and hyperplasia in occludin -/mice. Calcification in the brain, thinning of the bone and male infertility were also observed in occludin -/- mice (42). These data together suggested that occludin gene is indispensable and the complex phenotype seen its absence is probably due to an unidentified function of occludin. 

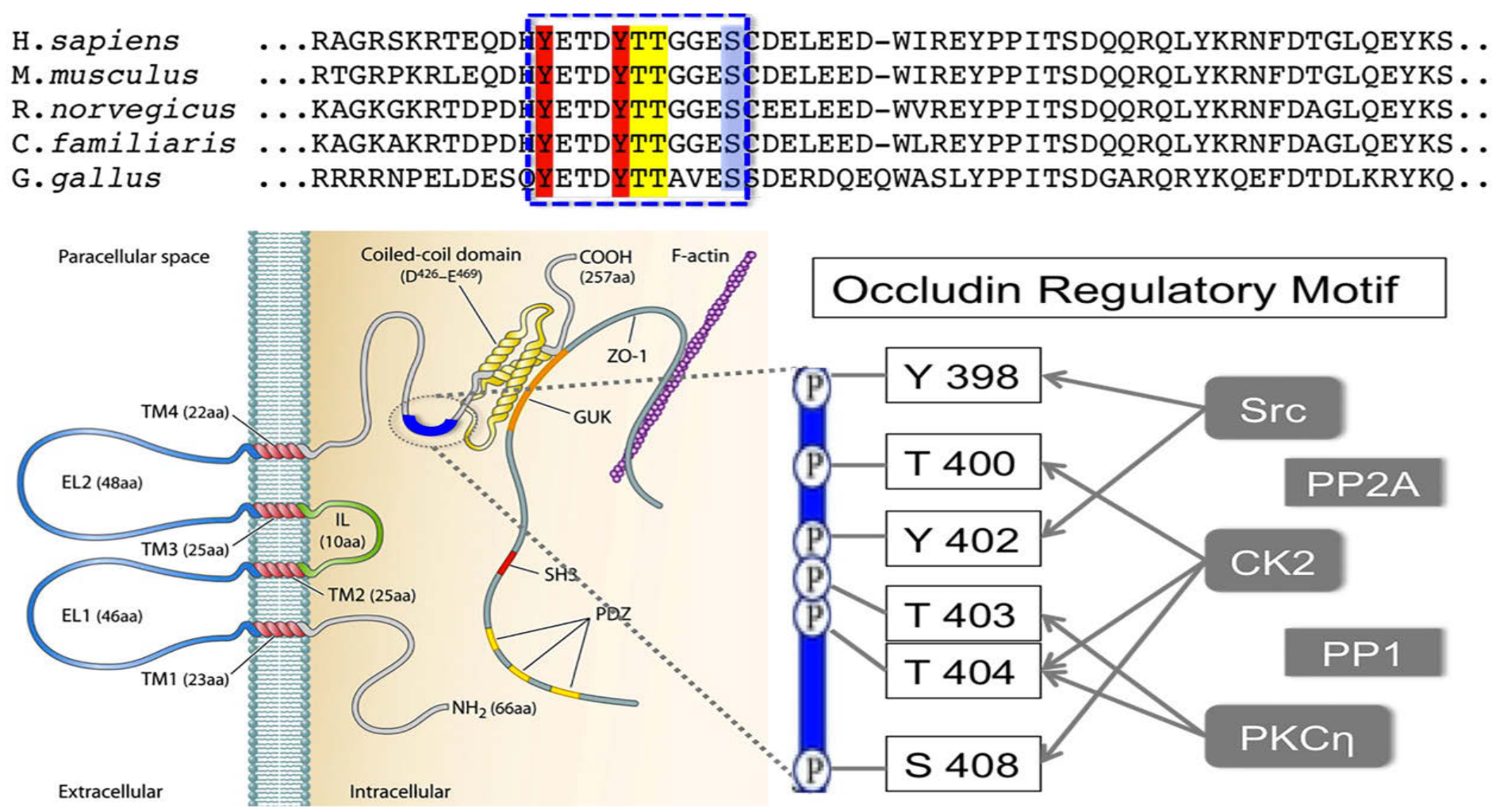

Figure 1-4. Conserved-phosphorylation Hot Spot in Occudin C-terminus.

A. Sequence alignment of a portion of Occludin C-terminus showing highly conserved region among various species. B. Domain structure of occludin with two extracellular loops (EL1 \& EL2), an intracellular loop (IL), four transmembrane domains(TM1-4), intracellular N and C-terminii. Distal region of occludin with phosphosites Y398-S408 is termed as occludin regulatory motif (ORM) Source: Reprinted with permission. Cummins, P. M. (2012). "Occludin: one protein, many forms." Mol Cell Biol 32(2): $242-250$. Copyright (C) 2012, American Society for Microbiology. 
Occludin was shown to form a complex with extrinsic death receptor and induce apoptosis in epithelial cells (43). Occludin downregulation by siRNA in human keratinocytes reduced cell-cell adhesion and susceptibility to apoptosis and altered $\mathrm{Ca}^{2+}$ homeostasis and cell differentiation (44). Occludin overexpression and knockdown altered TNF mediated changes in barrier function indicating that occludin is required for cytokine-mediated TJ regulation (45).

\section{TJ- a Dynamic Multi-Protein Complex at Steady State}

Studies by Turner group have demonstrated that the TJ multiprotein complex is highly dynamic at steady state. Extensive FRAP (Fluorescence recovery after photobleaching) based experiments were performed by Shen et al., in MDCK cell monolayers expressing EGFP tagged occludin/ZO-1/claudin-1/ $\beta$-actin (3). Fluorescence recovery analyses of each of these proteins revealed that most of the TJ-associated occludin is highly mobile (mobile fraction $71 \pm 3 \%$ ) with a slow exchange between bleached and fluorescent pools of occludin and a $t_{1 / 2}$ of $194 \pm 19 \mathrm{~s}$. ZO-1 mobility is similar to that occludin (mobile fraction $69 \pm 5 \%$ ) but exchanges more rapidly than occludin with a $t_{1 / 2}$ of $119 \pm 21 \mathrm{~s}$. Although occludin interacts with ZO-1, these two TJ proteins do not exchange as a complex and have distinct dynamic behavior. On the other hand, claudin-1 has relatively low mobility (mobile fraction $71 \pm 3 \%$ ) and hence only a small fraction of TJ associated claudin-1 is available for exchange. $\beta$-actin which is known to be highly dynamic, has a mobility that is higher than the three TJ proteins (mobile fraction $98 \pm 6 \%$ ) and $\mathrm{t}_{1 / 2}$ of $106 \pm 18$ (Table 1-1).

\section{Occludin Exchange Occurs by Diffusion within and Between TJ and Lateral Membrane}

In order to determine whether the diffusion during FRAP occurs within the membrane associated TJ or intracellular pools; further analyses by interfering with membrane fluidity, cellular ATP levels and vesicular transport were performed. Reducing the temperature of cell monolayers from $37^{\circ} \mathrm{C}$ to $14^{\circ} \mathrm{C}$, reduced the mobility of occludin. This was due to the low temperature mediated stabilization of cholesterol rich TJ membrane domains. Because endocytosis of occludin was shown to be involved in cytokine mediated $\mathrm{TJ}$ regulation, it was believed that fluorescence recovery of occludin was mediated by vesicular transport. But neither ATP depletion, nor treatment with a guanine exchange factor inhibitor affected occludin kinetics, suggesting that occludin exchange does not involve vesicular transport. On the other hand, methyl-B-cyclodextrin (MDCD)-mediated cholesterol depletion blocked occludin fluorescence recovery. FLIP (Fluorescence loss in photobleaching) analysis was used to assess the exchange between morphologically distinct protein pools of the TJ. FLIP analyses showed that continuous photobleaching of cytoplasm did not affect fluorescence loss at TJ, but photobleaching at the TJ resulted in fluorescence loss in the regions adjacent to the photobleached region. These data together suggest that occludin fluorescence recovery is mediated by diffusion within the membrane and the membrane fluidity plays an important role in its mobility. 
Table 1-1. Dynamic Behavior of TJ Proteins.

\begin{tabular}{lll}
\hline TJ Protein & Protein Pools & Value (\%) \\
\hline Occludin & Tight junction & 80 \\
& -Mobile & 80 \\
& -Immobile & 0 \\
& Lateral membrane & 20 \\
& -Mobile & 20 \\
& -Immobile & 0 \\
& Intracellular & 0 \\
ZO-1 & Tight junction & 40 \\
& -Mobile & 25 \\
& -Immobile & 15 \\
& Lateral membrane & 0 \\
& Intracellular & 60 \\
& Tight junction & 100 \\
Claudin-1 & -Mobile & 40 \\
& -Immobile & 60 \\
& Lateral membrane & 0 \\
& Intracellular & 0 \\
\hline
\end{tabular}

Source: Modified with permission. Shen, L., Weber, C. R., and Turner, J. R. (2008) The tight junction protein complex undergoes rapid and continuous molecular remodeling at steady state. J Cell Biol 181, 683-695. Copyright (C) 2008 Shen et al. 
$80 \%$ of occludin is present within the $\mathrm{TJ}$ while $20 \%$ is present at the lateral membrane. Interestingly, both these fractions are mobile. The confocal imaging of cells expressing EGFP-occludin shows occludin localized at TJ and also along the lateral membrane just below the TJ. The diffusion constant of occludin associated with TJ pool was found to be

$0.011 \mu \mathrm{m}^{2} \mathrm{~s}^{-1}$ while that of the occludin associated with the lateral membrane was found to be $0.1 \mu \mathrm{m}^{2} \mathrm{~s}^{-1}$. These data suggest that the diffusion of lateral membrane occludin is faster than that present at the TJ. Thus occludin exchange occurs by diffusion within and between $\mathrm{TJ}$ and lateral membrane.

\section{ZO-1 Exchanges with an Intracellular Pool}

Similar studies performed in cells expressing EGFP-ZO-1 showed that ZO-1 is highly mobile within the TJ. Cholesterol depletion by MBCD had no effect on ZO-1 mobility but depletion of cellular ATP and continuous photobleaching of cytoplasm reduced fluorescence recovery of ZO-1. These data together indicate that a large fraction of TJ associated ZO-1 exchanges with an intracellular pool in an energy dependent mechanism.

\section{Claudin-1 Exchange Occurs by Diffusion within the Membrane}

Claudin-1 on the other hand is less mobile as the fluorescence recovery is low after photobleaching. Continuous photobleaching of TJ resulted in rapid loss of claudin-1 fluorescence from junctions while that of cytoplasm had no effect. These data indicate that a small fraction of claudin-1 exchange occurs by diffusion within the TJ.

These observations strongly suggest that the $\mathrm{TJ}$ components are highly dynamic, and that such dynamic properties have the potential to regulate paracellular permeability via the fine-tuning of signaling cascades involved in barrier function (5). Understanding TJ dynamics will unravel TJ components as targets for the development of therapeutic agents aimed at modulating barrier function.

\section{TJ Regulation by Occludin Phosphorylation}

TJ regulation occurs in health as well as disease. Besides forming a seal that maintains the compartmentation, $\mathrm{TJ}$ serve to regulate the paracellular transport under various physiological and pathophysiological conditions. Several stimuli and signaling molecules have been reported to regulate the later function of TJ. Occludin is known to be an important target that mediates TJ regulation in these signaling cascades. TJ modulation by occludin phosphorylation and dephosphorylation has been studied extensively. The kinases and phosphatases involved are listed in (Table 1-2) Mutational analyses pointed towards ORM and its role in TJ modulation (Table 1-3). 
Table 1-2. Kinases and Phosphatases Associated with Occludin Phosphorylation.

\begin{tabular}{|c|c|c|c|}
\hline $\begin{array}{l}\text { Phosphatase } \\
\text { /Kinase }\end{array}$ & Experimental Model & Phosphosite & Reference \\
\hline$\overline{\mathrm{CK} 1}$ & $\begin{array}{l}\text { HUVEC } \\
\text { GST-Ocl-C (Human) }\end{array}$ & $\begin{array}{l}\text { Thr, Ser } \\
\text { Thr, Ser }\end{array}$ & $\begin{array}{l}(46) \\
(23)\end{array}$ \\
\hline CK2 & $\begin{array}{l}\text { Xenopus laevis embryo } \\
\text { GST-Ocl-C (Human) } \\
\text { Caco-2 }\end{array}$ & $\begin{array}{l}\text { Thr } 375 \text {, Ser } 379 \\
\text { Thr- } 400 / 404 \text {, Ser } 408 \\
\text { Ser } 408\end{array}$ & $\begin{array}{l}(47) \\
(38) \\
(37)\end{array}$ \\
\hline $\mathrm{c}-\mathrm{Src}$ & $\begin{array}{l}\text { Rat-1, MDCK } \\
\text { Caco-2 }\end{array}$ & $\begin{array}{l}\text { Tyr-398, Tyr-402 } \\
\text { Tyr }\end{array}$ & $\begin{array}{l}(34) \\
(33)\end{array}$ \\
\hline c-Yes & MDCK & Tyr & (48) \\
\hline $\mathrm{PKC \eta}$ & Caco-2, MDCK & Thr-403, Thr-404 & (35) \\
\hline PP2A & $\begin{array}{l}\text { GST-Ocl-C (Human), Caco-2 } \\
\text { MDCK }\end{array}$ & $\begin{array}{l}\text { Ser, Thr } \\
\text { Thr }\end{array}$ & $\begin{array}{l}(49) \\
(50)\end{array}$ \\
\hline PP1 & Caco-2, GST-Ocl-C (Chicken) & Ser & (49) \\
\hline DEP-1 & MDCK & Tyr & (51) \\
\hline PI3K & Caco-2, GST-Ocl-C (Chicken) & Tyr & (52) \\
\hline
\end{tabular}

Source: Reprinted with permission. Cummins, P. M. (2012). "Occludin: one protein, many forms." Mol Cell Biol 32(2): 242-250. Copyright (C) 2012, American Society for Microbiology. 
Table 1-3. Mutation Analyses of Phosphorylation Hotspot in Occludin.

\begin{tabular}{|c|c|c|c|c|}
\hline Kinase & Mutation & Effect on TJ & Model & Reference \\
\hline \multirow[t]{7}{*}{$\mathrm{c}-\mathrm{Src}$} & Y398/402D & TJ-assembly $\downarrow$ & Caco-2 & $(33,34,53)$ \\
\hline & & Inulin permeability $\uparrow$ & MDCK & \\
\hline & & Junctional occludin $\downarrow$ & & \\
\hline & & Interaction with $Z \mathrm{O}-1 \downarrow$ & & \\
\hline & Y398/402A & Junctional occludin $\uparrow$ & & \\
\hline & & TJ-assembly $\rightarrow$ & & \\
\hline & & Inulin permeability $\rightarrow$ & & \\
\hline \multirow[t]{5}{*}{$\mathrm{PKC \eta}$} & $\mathrm{T} 403 / 404 \mathrm{D}$ & TER $\uparrow$ & Caco-2 & $(35,54)$ \\
\hline & & Inulin permeability $\downarrow$ & MDCK & \\
\hline & $\mathrm{T} 403 / 404 \mathrm{~A}$ & TER $\downarrow$ & & \\
\hline & & Junctional occludin $\downarrow$ & & \\
\hline & & Junctional ZO-1 $\downarrow$ & & \\
\hline \multirow[t]{11}{*}{ CK2 } & S408A & Junctional occludin $\uparrow$ & Caco-2 & $(37,38,47)$ \\
\hline & & Interaction with ZO-1 $\uparrow$ & MDCK & \\
\hline & & Occludin exchange $\downarrow$ & $\mathrm{T} 84$ & \\
\hline & $\mathrm{S} 408 \mathrm{D}$ & Junctional occludin $\downarrow$ & & \\
\hline & & Interaction with ZO- $1 \downarrow$ & & \\
\hline & & Occludin exchange $\rightarrow$ & & \\
\hline & T404A/S408A & Occludin exchange $\downarrow$ & & \\
\hline & & Interaction with ZO- $1 \uparrow$ & & \\
\hline & & $\mathrm{TER} \uparrow$ & & \\
\hline & $\mathrm{T} 400 \mathrm{~A} / \mathrm{T} 404 \mathrm{~A} /$ & Interaction with ZO- $2 \downarrow$ & & \\
\hline & S408A & TJ-assembly $\downarrow$ & & \\
\hline
\end{tabular}

Source: Modified with permission. Dorfel, M. J. and O. Huber (2012). "Modulation of tight junction structure and function by kinases and phosphatases targeting occludin." J Biomed Biotechnol 2012: 807356. Copyright (C 2012 Max Johannes Dörfel and Otmar Huber. 


\section{Kinases}

c-Yes. Tyrosine kinase, c-Yes was pulled down with the peptide that mimics coiled-coil domain of occludin indicating a direct interaction between c-Yes and occludin (55). C-Yes induced-dephosphorylation of tyrosine residues during $\mathrm{Ca}^{2+}$ depletion mediated TJ disassembly in MDCK-II cells, was attenuated by c-Yes inhibitor CGP77675. Hence c-Yes was believed to be a barrier promoting kinase (48). However, the phosphosites in occludin for c-Yes were not identified.

c-Src. Oxidative stress induced tyrosine phosphorylation of occludin and redistribution of occludin and ZO-1 (56). Expression of a kinase inactive c-Src delayed oxidative stress induced TJ disruption indicating that c-Src was the potential tyrosine kinase involved. c-Src was found to interact with GST tagged occludin c-terminus in vitro which decreased interaction between occludin and ZO-1 (33). Y398 and Y402 present in a highly conserved motif YETDYTT were identified as the phosphosites for cSrc (34). A phosphomimetic mutation of Y398/402D in occludin attenuated its interaction with ZO-1, delayed TJ reassembly during $\mathrm{Ca}^{2+}$ switch in MDCK cells and sensitized them to hydrogen peroxide induced barrier dysfunction (34).

FAK. Focal adhesion kinase plays a key role in the regulation of cytoskeleton, cell migration and adhesion. Acetaldehyde treatment in human colonic biopsies resulted in the activation of FAK, increased tyrosine phosphorylation of occludin and ZO-1 and redistribution of occludin from intercellular junctions. EGF or glutamine pretreatment was able to prevent these effects.

PKC $\eta$. Over expression of constitutively active PKC $\eta$ in Caco-2 and MDCK cells enhanced barrier function by increasing TER, decreasing inulin-flux and increased occludin localization at the intercellular junctions. These effects were blocked by the use of PKC $\eta$ pseudosubstrate and shRNA-induced knockdown of PKC $\eta$. PKC $\eta$ was shown to interact with occludin. By mass spectrometry and mutational analyses, T403 and T404 were identified as the phsophosites for PKC $\eta$. T403A mutation partially blocked phosphorylation whereas T404A completely prevented it. T403/404D mutation enhanced occludin and ZO-1 localization at the intercellular junctions while T403/404A mutation decreased ZO-1 localization at junctions and occludin was seen mostly in the intracellular compartment (35).

PKC $\zeta$. Atypical PKC like was shown to interact with occludin $\mathrm{C}$-terminus and phosphorylate T403, T404, T424 and T438. Inhibition of PKC $\zeta$ using pseudosubstrate led in MDCK and Caco-2 cell led to decrease in TER, increase in permeability, decreased occludin phosphorylation, redistribution of occludin from the junctions and delayed $\mathrm{Ca}^{2+}$ 
induced TJ reassembly. Mutation of T424/438 to alanine resulted in occludin localization in intracellular compartment and delayed $\mathrm{Ca}^{2+}$ induced $\mathrm{TJ}$ reassembly (57).

CK2. Casein kinase 2 is a tetrameric serine/threonine kinase with two catalytic subunits(a,a') and two regulatory b-subunits. In vitro phosphorylation studies revealed that CK2 interacts with occludin and phosphorylates T400, T404 and S408. A triple mutant T400A/T404A/S408A blocked complete phosphorylation of occludin in vitro. In Caco-2 cells, knockdown of CK2 or pretreatment with CK2 inhibitors enhanced barrier function by increasing TER and decreasing paracellular flux of sodium ions. FRAP studies using occludin knockdown-Caco-2 cells expressing EGFP-occludin showed that CK2 inhibition reduced mobile fraction of occludin. Mutants S408A and T404A/S408A showed decreased mobility compared to wild type occludin, S408D and T404D/S408D mutants (38). It was postulated that S408 phosphorylation might act as a switch that regulates TJ permeability by determining whether occludin forms homodimers or heteromeric interactions with ZO-1, claudin-1 and claudin-2 (37).

PI3K. Phosphatidyl inositol-3 kinase, regulatory subunit p85 was found to directly interact with coiled-coil domain of occludin c-terminus (55). Oxidative stress induces translocation of $\mathrm{p} 85$ to the junctions and increased association with occludin. Treatment of T84 cell monolayers with LY294002, a PI3K inhibitor, attenuated tyrosine phosphorylation of occludin, decrease in TER, increase in permeability and redistribution of occludin and ZO-1 from the junctions (52). Hence it was believed that PI3K activity is involved in dissociation of TJ during oxidative stress.

\section{Phosphatases}

PP2A and PP1. Protein phosphatase 2A and 1 (PP2A and PP1) are serine/threonine phosphatases ubiquitously expressed and associated with multiple functions. In MDCK cells, enhanced PP2A activity induced dephosphorylation of occludin and increased paracellular permeability which was attenuated by pretreatment with okadaic acid, a PP2A inhibitor (50). In Caco-2 cells, inhibition of PP2A activity by okadaic acid and fostriecin, resulted in enhanced TJ reassembly after $\mathrm{Ca}^{2+}$ switch. SiRNA-mediated reduction of PP2A activity enhanced $\mathrm{Ca}^{2+}$ induced increase in TER, decrease in inulin permeability and enhanced junctional localization of occludin and ZO1 during TJ reassembly. By in vitro assays it was found that PP2A dephosphorylates phospho-occludin on threonine residues while PP1 dephosphorylates on serine residues. Both PP2A and PP1 were found to interact with occludin C-terminus and negatively regulate TJ assembly (49).

PP2A was also shown to be involved in hydrogen peroxide induced TJ disruption in Caco-2 cell monolayers. Hydrogen peroxide enhanced association between occludin and PP2A in Caco-2 cells, which was blocked by a SC kinase inhibitor, PP1 suggesting 
that this interaction is a tyrosine kinase dependent mechanism (58). Acetaldyde induced TJ disruption also involves dephosphorylation of occludin by PP2A in Caco 2 cell monolayers. Acetaldehyde treatment increased translocation of PP2A to TJ and induced threonine dephosphorylation of occludin (59).

DEP1. (Density-enhanced protein tyrosine phosphatase-1) has been shown to interact with occludin in MDCK-II cells. Overexpression of DEP-1 in these cells led to increased TJ reassembly and enhanced barrier function. Knockdown of DEP-1 resulted in increased permeability (51).

\section{Cytokine-induced TJ Regulation Involves Occludin}

Modulation of TJ without altering TJ protein expression has been shown to occur during physiological regulation of TJ by SGLT-1 (Sodium glucose transporter-1) and pathophysiological regulation of TJ by proinflammatory cytokines like TNF and IL1- $\beta$. Myosin light chain kinase (MCLK) is the key player underlying these processes. Na+glucose cotransport activation leads to MLCK mediated phosphorylation of MLC. This leads to perijunctional actomyosin contraction and increased paracellular permeability. TNF also induces activation of MLCK, which in turn induces actomyosin condensation by MLC phosphorylation and increases paracellular permeability. Exposure to T-cell derived cytokines in intestinal epithelial cells leads to caveolin mediated endocytosis of occludin as a result of MLCK activation. This effect was blocked by inhibition of MLCK as well as by drugs that block caveolar pathway.

In mice expressing EGFP-tagged occludin, exposure of the intestinal epithelium to TNF induced focal accumulation of occludin, which was blocked by inhibitors of caveolar pathway. These data suggested that TNF induced TJ regulation involves endocytosis of occludin. In HT-29/B9 cells, TNF and interferon-gamma exposure diminished occludin promoter activity, which resulted in enhanced paracellular permeability associated with occludin downregulation (60).

\section{VEGF}

Vascular endothelial growth factor (VEGF) is known to be an important mediator of vascular permeability and $\mathrm{TJ}$ deregulation in blood-brain and blood-retinal barriers. VEGF was found to induce phosphorylation of S490 of occludin (61). This enhances interaction with E3 ubiquitin ligase and induces occludin ubiquitination. VEGF induced occludin phosphorylation in BRECs resulted in increased permeability and decreased TER (30). Thus VEGF alters TJ and increases vascular permeability in brain and retinal disorders. Human intestinal adenocarcinoma was shown to exhibit decreased occludin expression and tumor derived VEGF is known to play an important role in occludin degradation and tumor metastasis (28). 


\section{Occludin Variants}

Occludin is known to exhibit functional diversity by means of its variants generated by gene splicing and alternate promoter usage. Saitou et al (62) were the first to report isoforms of occludin, generated by alternative splicing.

\section{Occludin with N-terminus Extension}

Occludin 1B, originally identified in MDCK cells is an alternatively spliced variant with a 193-bp insertion that encodes an occludin isoform with a 56 amino acid $\mathrm{N}$ terminal extension (63). This was later found to be expressed in human colon carcinoma cells and mouse intestine. It was known to localize at the junctions similar to wild type occludin. The extended N-terminus of occludin 1B was believed to mask the SH3 motif and hinder binding interactions with $\mathrm{SH} 3$ adaptor proteins.

\section{Occludin with TM4 Deletion}

TM4 (-), identified in human tissues, embryos, epithelial and endothelial cells, is an isoform generated by skipping exon 4 leading to a 162-bp deletion that encodes TM4 and proximal c-terminus region. TM4 (-) also referred to as type II occludin, failed to localize at the junctions because of the externalized C-terminus, and does not colocalize with ZO-1. It was believed that low-level expression of TM4 (-) occurs during tissue remodeling,

\section{Occludin with Alternate Promoter (Exon 1a)}

This variant was known to be generated using second occludin promoter and transcription start site (exon 1a). It has enhanced sensitivity TNF signaling and believed to have a pathological role inducing colonic inflammation in ulcerative colitis.

\section{Occludin with Alternate Promoter and Exon 9 Deletion}

$\operatorname{Occ} \Delta^{\mathrm{E} 9}$ was identified in human liver cells. This isoform localized in the cytoplasm and failed to induce apoptosis like the canonical occludin and hence was speculated to have a role in metastasis.

\section{Significance}

Although the studies so far did not confirm the functional significance of these occludin variants, it is strongly believed that they are expressed under conditions of physiological regulation of TJ or during disease pathogenesis and cancer progression. 


\section{Occludin in Disease Pathogenesis}

Occludin is an important target during barrier breach and hence is associated with several disease processes. In all these cases occludin is subjected to either downregulation or altered subcellular localization.

\section{Diarrhea}

Pathogenic toxins that target gastro intestinal tract mediate disease pathogenesis by mechanisms that involve occludin and increase in paracellular permeability. Listed below are some of the examples.

a. Gliadin exposure to intestinal biopsies from celiac patients increased permeability to macromolecules by Zonulin release and loss of occludin-ZO1 interaction (64).

b. Enteropathogenic Escherichia coli (EPEC) secretes a protein called EspF that increases intestinal permeability, reduces TER and dissociates occludin from TJ by inducing dephosphorylation (65).

c. Clostridium perfringens enterotoxin (CPE) forms a large protein complex with occludin that increases permeability and induces cytotoxicity in Caco-2 cells (66).

d. Vibrio cholera produces a cytotoxin, hemagglutinin/protease (HA/P) that causes barrier dysfunction by degrading occludin and perturbing actin cytoskeleton in MDCK-I cells. This leads to decrease in TER and loss of TJ strands (67).

e. Clostridium difficile toxins A and B are UDP-glucosyltransferases that glucosylate and inactivate Rho. This induces cytoskeletal disorganization, dissociation of occludin and ZO-1 from TJ and increases TJ permeability in T84 intestinal epithelial cells (68).

\section{Inflammation}

TJ barrier dysfunction associated increase in epithelial permeability is believed to either predispose or enhance disease progression. Crohn's disease and ulcerative colitis are collectively referred to as inflammatory bowel disease (IBD). Patients with IBD exhibit increased intestinal paracellular permeability and decreased occludin expression. IBD pathogenesis involves production of a cytokine like tumor necrosis factor (TNF). MLCK expression and activity were also enhanced in intestinal epithelium of IBD patients. Thus TNF induced occludin downregulation and occludin endocytosis by MLCK activation are the key mechanisms underlying barrier dysfunction mediated disease progression. 


\section{Cancer}

TJ between cells confer cell-cell adhesion and maintain integrity of the epithelium. Hence it is believed that the TJ disassembly is required for the cells of neoplastic epithelium to metastasize. The process of metastasis involves TJ disruptionmediated detachment of tumor cell from its primary site, intravasation through the endothelial barrier often induced by secretion of growth factors like VEGF, HGF and extravasation of the circulating tumor cell leading to invasion of other tissues. The following are the instances where occludin down regulation induced TJ dysfunction aids in metastases of various tumors.

a. Tumors of rat and human colon showed increased permeability while histological analysis of human intestinal adenocarcinomas showed reduced expression of occludin. A correlation between tumor differentiation and occludin expression was reported (69).

b. Hepatocyte growth factor or scatter factor (HGF/SF), a cytokine released by stromal cells is known to increase paracellular permeability, decrease TER and alter occludin expression in human breast cancer cell lines. Down regulation of occludin is believed to play a role in metastasis of breast cancer cells (70).

c. In human endometrial cancer, with the increase in carcinoma grade, expression of occludin was found to be decreased. Decreased occludin expression was also found during invasion of the myometrium and lymph node metastasis (71).

\section{Alcoholic Endotoxemia}

Alcohol increases paracellular permeability to endotoxins in the gut. Alcohol induced endotoxemia is involved in the pathogenesis of alcoholic liver disease (ALD) (72). Ethanol and its metabolite acetaldehyde induced TJ and AJ disruption in Caco-2 cell monolayers. Acetaldehyde treatment in these cells induced occludin redistribution from intercellular junctions. This involved dephosphorylation of occludin on threonine residues mediated by PP2A (59). In a recent study using occludin knock out mice, we showed that lack of occludin exacerbates chronic ethanol-induced increase in colonic mucosal permeability. Similarly, using shRNA mediated occludin knock down, acetaldehyde exposure in Caco- 2 cell monolayers was shown to enhance TJ disruption and barrier dysfunction (73). These data suggest that occludin plays an important role in barrier function and that its disruption sensitizes TJ to alcohol induced endotoxemia.

\section{Hepatitis}

Human occludin was identified as an important entry factor for hepatitis $\mathrm{C}$ virus (HCV) infection. Occludin silencing impaired infection of lentiviral particles bearing HCV glycoprotein (HCVpp) (74). A later study showed that occludin interacts with HCV 
glycoprotein (HCVgp). Occludin knock down led to reduced infection by cell culture derived HCV particles. Thus occludin plays an essential role in HCVgp fusion at late entry events of $\mathrm{HCV}$ infection (75). A recent study showed that $\mathrm{HCV}$ infection in hepatoma cells induced an increase in occludin expression and upregulation of adiposedifferentiation related protein. (ADRP). In HCV-infected human livers, ADRP mRNA expression levels correlated with that of occludin

\section{Miscellaneous}

a. Oncogenic Raf1 mediated tumorigenesis causes downregulation of occludin in rat salivary gland epithelial cells. The second extracellular loop of occludin is known to be required for inhibition of Raf-1 mediated tumorigenesis in vitro and in vivo $(76,77)$.

b. Ras-transformed MDCK cells have loss of occludin and ZO-1 from TJ. This increased TJ permeability is known to involve MAPK signaling pathway. Ras mutations are known to cause barrier dysfunction by altering occludin and claudin-1 levels at TJ (78).

c. Rho Kinase (RhoK) activation mediated occludin phosphorylation is associated with barrier leakiness in the case of human immunodeficiency virus encephalitis (HIVE) (79). Malignant brain tumors also displayed leaky endothelial TJ and loss of occludin expression (80).

d. Helicobacter pylori sonicates are known to increase gastric permeability by altering expression of occludin in a PKC dependent mechanism (28).

e. A discontinuous staining pattern of occludin in blood brain endothelium is considered a hallmark of HIVE (HIV associated encephalitis) (79).

f. Serum from multiple sclerosis patients induced downregulation of occludin in cultured endothelial cells (81).

\section{Hypothesis and Specific Aims}

Epithelial TJ serve as a critical intercellular barrier against paracellular permeation of luminal antigens, toxins and pathogens. TJ disruption-induced increase in paracellular permeability leads to edema, jaundice, enterocolitis, alcoholic endotoxemia, IBD, celiac disease and colon cancer. Hence understanding the dynamic nature of TJ barrier is crucial to determine their physiological role in normal development and during pathogenesis.

$\mathrm{TJ}$ is known to be a highly dynamic multi-protein complex with its components occludin, claudins and ZO-1, each exhibiting a unique dynamic behavior. Yet, the 
contributing factors for this dynamic nature remain elusive. It is well accepted that occludin phosphorylation in a highly conserved region (Tyr398-Ser408), which we call $\mathrm{ORM}$, plays a role in $\mathrm{TJ}$ regulation. However, the function of this motif in $\mathrm{TJ}$ dynamics is not well defined.

\section{Hypothesis}

Based on the above observations, we hypothesized that ORM confers dynamics to epithelial $\mathrm{TJ}$ and regulates barrier function by ORM-mediated protein-protein interactions. Our goal is to investigate the functional significance of ORM and ORMmediated signaling mechanisms that underlie $\mathrm{TJ}$ regulation. This goal will be achieved by conducting studies under the following specific aims.

\section{Specific Aim 1: To Determine That ORM Confers Dynamics to TJ in a Phosphorylation-dependent Mechanism}

Studies designed here were aimed to determine the function of ORM in TJ dynamic using a deletion mutant of occludin that lacks ORM. Furthermore, double pointmutants of occludin phosphosites were used to establish the significance of ORM mediated phosphorylation in TJ dynamics.

\section{Specific Aim 2: To Determine That ORM-mediated Protein Interactions Are Involved in TJ Modulation}

Studies designed here were aimed to identify the proteins that interact with ORM, using a synthetic peptide that mimicked ORM. Furthermore, the functional significance of ORM-mediated protein interactions was established in relation to TJ modulation. 


\title{
CHAPTER 2. MATERIALS AND METHODS
}

\author{
Cell Culture and Transfection
}

\section{Cell Lines}

Madin-Darby canine kidney (MDCK) cell line was originally derived from kidney tissue of a normal adult female dog. These cells are known to exhibit apico-basolateral polarity and well-defined cell junctions. Hence they have been widely used to study tight junctions, protein trafficking and polarity associated with epithelial cells. MDCK cells were purchased from American Type Cell Culture (ATCC). They were grown in Dulbecco's modification of eagle's medium (DMEM) supplemented with $10 \%(\mathrm{v} / \mathrm{v})$ fetal bovine serum (FBS), $1 \mathrm{mg} / 10 \mathrm{ml}$ primocin and maintained at $37^{\circ} \mathrm{C}$ and $5 \%$ carbon dioxide $\left(\mathrm{CO}_{2}\right)$.

Caco-2 cells were isolated from adenocarcinoma of a 72-year old Caucasian male. These cells after differentiation are known to express brush border enzymes and microvilli on their apical membrane. Caco- 2 cells are widely used to study intestinal permeability and cell signaling, as they closely resemble the intestinal epithelium when grown on polycarbonate transwell inserts. Hence this cell line is treated as an ideal model to study intestinal barrier function. Caco-2 cells were purchased from American Type Cell Culture (ATCC). They were grown in Dulbecco's modification of eagle's medium (DMEM) supplemented with $20 \%$ (v/v) fetal bovine serum (FBS), 1mg/10ml primocin and maintained at $37^{\circ} \mathrm{C}$ and $5 \%$ carbon dioxide $\left(\mathrm{CO}_{2}\right)$.

\section{Occludin Gene Silencing}

For occludin gene silencing in canine MDCK cells, vector-based short hairpin RNA (shRNA) was designed using the Dharmacon Web site (siDesign ${ }^{\circledR}$ Center, http://www.dharmacon.com/DesignCenter/) and cloned into pRNAtin-U6 vector (GenScript). Two targeting sequences were chosen against the nucleotide sequence of canine occludin gene using the Dharmacon web site (Target1:

TATGTCAGACCTTATAACG; Target2: TATGCTACCACCCATTAAG). The sequences were further verified by BLAST search on the known human genome database, and no matches were found confirming the uniqueness of these sequences.

To construct the shRNA vectors, two pairs of oligonucleotides containing the antisense sequence, a hairpin loop region (TTGATATCCG), and the sense sequence with cohesive BamHI and XhoI sites were synthesized (Sigma Genosys, St Louis, MO) using the primers provided in the table (Table 2-1). The top and bottom strands were annealed and cloned into BamHI and XhoI sites of the pRNAtin-U6 vector (GenScript Corp., Piscataway, NJ) (pR vector), which induces expression of shRNA by U6 promoter. Successful insertion of the shRNA constructs into the vector was confirmed by releasing 
Table 2-1. Primers for Knockdown and Mutation of Occludin.

\begin{tabular}{ll}
\hline Primer & Sequence \\
\hline cOCN-shRNA1-Top strand & GATCCCGTATGTCAGACCTTATAACGTTGA \\
& TATCCGCGTTATAAGGTCTGACATATTTTTT \\
& CCAAA \\
cOCN-shRNA1-bottom strand & AGCTTTGGAAAAAATATGTCAGACCTTAT \\
& AACGCGGATATCAACGTTATAAGGTCTGAC \\
& ATACGG \\
cOCN-shRNA2-Top strand & GATCCCGTATGCTACCACCCATTAAGTTGA \\
& TATCCGCTTAATGGGTGGTAGCATATTTTT \\
& TCCAAA \\
cOCN-shRNA2-bottom strand & AGCTTTTGGAAAAATATGCTACCACCCAT \\
& TAAGCGGATATCAACTTAATGGGTGGTAGC \\
& ATACGG \\
A388-415 Forward & TGGATCAGGGAATATCCACCTATCA \\
$\Delta 388-415$ Reverse & TGCTCTTCCCTTTGCAGGTGCTCT \\
Y398/402A & CAGAGCAAGATCACGCTGAGACAGACGC \\
Y398/402D & CACAACTGGCGGCGAG \\
& CAGAGCAAGATCACGATGAGACAGACGA \\
T403/404A & CACAACTGGCGGCGAG \\
T403/404D & CAAGATCACTATGAGACAGACTACGCAGC \\
T400/403/404A & CTATGATCACTATGAGACAGACTACGATG \\
& ATGGC \\
T400/403/404D & GATCACTATGAGGCAGACTACGCAGCTGG \\
& CGGCG \\
\hline
\end{tabular}

Note: $\mathrm{pEGFP}$ occludin wild type construct was used as the template for all mutations except T403/404A and T403/404D, where templates were T400/403/404A and T400/403/404D respectively. 
the oligonucleotides by digesting with BamHI and XhoI and sequencing. For a control, a mutant form of shRNA was designed by replacing adenine and guanine nucleotides with cytosine and adenine, respectively.

\section{ORM Deletion Mutation}

ORM deletion mutant was generated in a pEGFP-human occludin construct by inverse PCR using primers flanking the ORM region. The PCR product was treated with DPn1 to digest the plasmid DNA and a self-ligation was performed by a reaction with T4 polynucleotide kinase and T4 DNA ligase (New England Biolabs Inc., Beverly, MA).

The mutation was confirmed by restriction digestion, PCR amplification and sequencing. The mutant construct was transformed into XL10 gold competent cells for DNA amplification. This resulted in a deletion mutant of occludin $\left(\mathrm{Ocl}^{\mathrm{DM}}: \Delta^{388-415}\right)$ lacking 28 amino acids (GRSKRTEQDHYETDYTTGGESCDELEED) in its C-terminus.

\section{Transfection}

Stable clone of occludin knock down MDCK cell line (OD-MDCK) generated by shRNA transfection were grown in Dulbecco's modified Eagle's medium supplemented with $10 \%$ fetal bovine serum (Gibco), primocin (100ug/ml; Invivogen) and hygromycin (100ug/ml; Thermo Fischer). The constructs pEGFP-occludin ${ }^{\mathrm{WT}}$, pEGFP-occludin ${ }^{\mathrm{DM}}$ or vector alone were transfected into OD-MDCK cells using lipofectamine 2000 (Invitrogen). Stable clones of OD-MDCK ${ }^{\mathrm{WT}}$, OD-MDCK ${ }^{\mathrm{DM}}$ and OD-MDCK-Vec were generated by G418 mediated selection and dilution cloning. At least 12 clones from each group were screened for GFP fluorescence by live-cell confocal imaging and protein expression by western blot analysis.

\section{Fluorescence Microscopy}

\section{Immunofluorescent Staining}

Cell monolayers or tissue cryosections were fixed in either acetone/ methanol $(1: 1)$ at $0{ }^{\circ} \mathrm{C}$ for $5 \mathrm{~min}$ or $3 \%$ paraformaldehyde (for staining actin-cytoskeleton) at room temperature for $15 \mathrm{~min}$. Those fixed in acetone/methanol were stored at $-20^{\circ} \mathrm{C}$ while those fixed in paraformaldehyde were stored in PBS at $4{ }^{\circ} \mathrm{C}$ for not more than a week. On the day of staining, fixed monolayers or cryosections were washed twice with PBS and permeabilized in $0.2 \%$ Triton X-100 for $10 \mathrm{~min}$. After washing three times with PBS, they were blocked using 4\% milk in TBST (20mM Tris, $150 \mathrm{mM} \mathrm{NaCl}, \mathrm{pH} 7.2$ ) for $30 \mathrm{~min}$ at room temperature. Then they were incubated with primary antibodies in $4 \%$ milk (antiZO-1, anti-GFP, anti-occludin, anti-MAP7, anti- $\beta$-tubulin, anti-E-Cadherin, anti- $\beta$ catenin) for 1 hour at room temperature. The monolayers were washed with $1 \%$ milk followed by incubation with secondary antibodies (Alexa Fluor 488-conjugated anti- 
mouse and Cy3-conjugated anti-rabbit $\operatorname{IgG}$ ) in 4\% milk. Nuclear staining was done using Hoechst 33342. F-actin and biotin were stained with AlexaFluor-488 conjugated phalloidin and $\mathrm{Cy} 3$ conjugated streptavidin respectively. The monolayers were mounted on a slide using mounting fluid and sealed for imaging.

\section{Microscopy}

- Fluorescence was visualized in a Zeiss LSM 5 laser scanning confocal microscope. The images from Z-series sections (1 $\mu \mathrm{m}$ thickness) were collected by Zen software. The $\mathrm{z}$-series images were stacked by using the software, Image $\mathrm{J}$ (National Institutes of Health), and processed by Adobe Photoshop (Adobe Systems).

- For time-course imaging studies, confluent cell monolayers grown in $60 \mathrm{~mm}$ culture plates were imaged using a $20 \mathrm{X}$ objective of the confocal microscope. Cells were maintained in an incubator at $37^{\circ} \mathrm{C}$ during the course of the experiment. For cells in chamber slides, imaging was done by Nikon epifluorescence microscope using a 40X objective.

\section{Analysis of Barrier Function and TJ Integrity}

\section{Barrier Function Parameters}

Cells were grown in transwell inserts $(12 \mathrm{~mm}$; Costar) to achieve confluence at a density of $3 \times 10^{5}$ cells per well. The experiments were performed on days 1-4 in MDCK cells and on days 7-10 in Caco-2 cells. Barrier function of the monolayers was monitored by measuring electrical resistance of the barrier and unidirectional flux of FITC-inulin.

- Trans-epithelial Electrical Resistance (TER) is a measure of the flux of all ions across the epithelium. TER was measured by using a Millicell-ERS Electrical Resistance System (Millipore, Bedford, MA). Basal TER of semi-permeable membrane of transwells was subtracted from all values $(80-100 \mathrm{Ohms} / \mathrm{cm} 2)$.

- Unidirectional FITC-inulin flux. FITC-inulin $(6 \mathrm{kDa} ; 0.5 \mathrm{mg} / \mathrm{ml})$ was administered to the buffer in the apical well. At the end of incubation, $100 \mu \mathrm{l}$ of basal medium and $20 \mu \mathrm{l}$ of apical medium were sampled, and fluorescence was measured using an Flx-800 microplate fluorescence reader (BioTEK instruments, Winooski, VT). Flux into the basal well was calculated as the percentage of total apical well fluorescence/cm2 surface area. 


\section{TJ Integrity}

Cell monolayers were fixed in acetone/ methanol (1:1) for staining occludin, GFP, ZO-1, E-cad, b-catenin and cld-2 while cells were fixed in 3\% paraformaldehyde for staining actin and tubulin. TJ integrity of the monolayers was analyzed by confocal microscopy.

\section{FRAP}

FRAP (Fluorescence recovery after photo-bleaching) studies were performed using a module available in the Zen software. The principle involved in FRAP is represented in the schematic (Figure 2-1). Cells stably expressing GFP-tagged occludin and its mutants were grown to confluence in $60 \mathrm{~mm}$ culture plates. During imaging, cell monolayers were washed and placed with DMEM without glutamine and phenol red. 3-4 regions of interest (ROI), provided in the module were defined at the intercellular junctions of the monolayer. High intensity laser was used at $488 \mathrm{~nm}$ to bleach the ROI, with a specific number of iterations. The module was set to capture at least 3 images before the bleach and time-lapse images up to $\sim 25 \mathrm{~min}$ after the bleach.

All the images were collected at the same focal plane. The time series images were processed by using ImageJ software and confirmed by manual calculation. Fluorescent intensity was measured at varying times after bleaching to calculate percent recovery. Percent recovered at $25 \mathrm{~min}$ was considered as percent mobile fraction.

\section{Protein Analyses}

\section{Preparation of Triton-soluble and Triton-insoluble Fractions}

Cell monolayers in $60 \mathrm{~mm}$ culture plates were incubated with $500 \mu \mathrm{l}$ lysis bufferCS (Tris buffer containing protease inhibitor cocktail $(10 \mathrm{ul} / \mathrm{ml}), 1 \mathrm{mM}$ vanadate, and 1 $\mathrm{mM}$ PMSF) on ice for $15 \mathrm{~min}$. Cell lysates were centrifuged at $15,600 \mathrm{~g}$ for $4 \mathrm{~min}$ at $4^{\circ} \mathrm{C}$ to sediment the high-density actin cytoskeleton. The supernatant was used as the tritonsoluble fraction.

The pellet that is known to contain membrane associated TJ components, was suspended in $200 \mu \mathrm{l}$ of lysis buffer-CS and sonicated to homogenize the actin cytoskeleton. This was used as triton-insoluble fraction. Protein contents in both fractions were measured by the BCA method (Pierce Biotechnology, Rockford, IL). The protein fractions were mixed with equal volume of $2 \mathrm{X}$ Laemmli's sample buffer and heated at $100^{\circ} \mathrm{C}$ for $10 \mathrm{~min}$. 

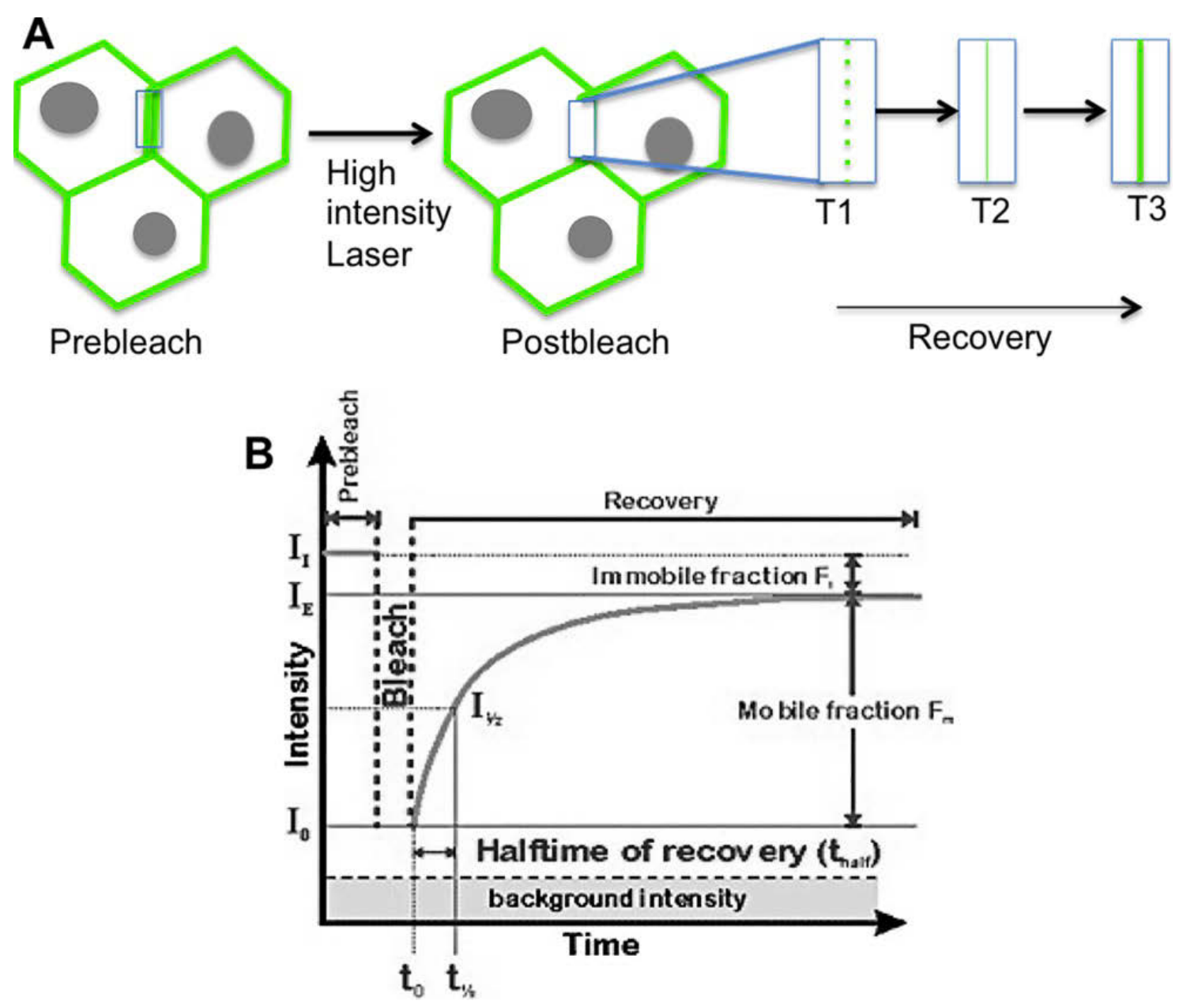

Figure 2-1. Fluorescence Recovery after Photobleaching (FRAP).

A. Live-cell images of a confluent monolayer are captured before photobleaching. A high intensity laser is used to bleach a specified region of interest (ROI) at intercellular junctions. Time-lapse images post bleaching are captured until the fluorescence intensity reaches a plateau. B. A plot of fluorescence intensity (I) versus time ( $t$ ) gives a measure of mobile $\left(\mathrm{F}_{\mathrm{m}}\right)$ and immobile fractions $\left(\mathrm{F}_{\mathrm{I}}\right.$ and half time of recovery $\left(\mathrm{t}_{\text {half }}\right)$. 


\section{Immunoprecipitation}

Confluent monolayers (60mm culture plates) were washed with ice-cold PBS and proteins were extracted in 500ul ice-cold lysis buffer $(10 \mathrm{mM}$ Tris/Cl pH 7.5 containing $150 \mathrm{mM} \mathrm{NaCl}, 0.5 \mathrm{mM}$ EDTA, 0.5\% NP40, 1mM PMSF, 1x Protease inhibitor cocktail). The lysates were centrifuged at $15600 \mathrm{~g}$ for $4 \mathrm{~min}$ at $4{ }^{\circ} \mathrm{C}$. The supernatant was separated and the pellet was resuspended in $200 \mathrm{ul}$ lysis buffer and sonicated for $10 \mathrm{sec}$ to induce fragmentation of F-actin filaments and release actin-bound TJ proteins. Previous studies showed that nearly $80 \%$ of the occludin and ZO-1 was recovered in this fraction. This was centrifuged at $13500 \mathrm{rpm}$ for $10 \mathrm{~min}$ and the supernatant was pooled with the previous. The protein concentration was quantitated by BCA method. The total lysates $(1.0 \mathrm{mg}$ protein $/ \mathrm{ml})$ were incubated with prewashed $25 \mathrm{ul} \mathrm{GFP-Trap \_ A} \mathrm{beads}$ (Chromotek, Germany) at $4{ }^{\circ} \mathrm{C}$ for $1 \mathrm{~h}$. The immune complexes were isolated by centrifugation at $2000 \mathrm{~g}$ for $2 \mathrm{~min}$ at $4{ }^{\circ} \mathrm{C}$, followed by washes with Tris buffer and suspended in equal volume of Laemelli sample buffer $(2 \mathrm{x}$ concentrated). The protein was extracted by heating the samples at $100{ }^{\circ} \mathrm{C}$ for $10 \mathrm{~min}$ and centrifuging at $13500 \mathrm{rpm}$ for $10 \mathrm{~min}$. For immunoprecipitation of $\mathrm{p}$-Thr, lysates were heated in the presence of $0.3 \%$ SDS for $10 \mathrm{~min}$ at $100{ }^{\circ} \mathrm{C}$ followed by centrifugation. The clear supernatant was then used for immunoprecipitation.

\section{Immunoblot Analysis}

Protein samples were separated by 7\% SDS-polyacrylamide gel electrophoresis and transferred to polyvinylidene diflouride (PVDF) membranes. Membranes were blotted for occludin, ZO-1, GFP, actin, claudin-2, Peroxiredoxin 1 and MAP7 using specific primary antibodies in combination with secondary HRP-conjugated anti-mouse IgG or HRP-conjugated anti-rabbit IgG antibodies. GST was immunoblotted using HRPconjugated anti-GST antibody. The blots were developed using ECL chemi-luminescence method (Amersham, Arlington Heights, IL).

\section{$\mathrm{Ca}^{2+}$ Switch Assay}

Cells expressing GFP-Ocl ${ }^{\mathrm{WT}}$ and its mutants were grown to confluence in transwells or $60 \mathrm{~mm}$ culture plates. The principle involved in $\mathrm{Ca}^{2+}$ switch assay is represented in the schematic (Figure 2-2). LCM was prepared from $\mathrm{Ca}^{2+}$ free DMEM (Thermo Fisher Scientific, Tustin, CA.) supplemented with $1 \mathrm{mM}$ Sodium pyruvate, 2 $\mathrm{mM}$ glutamine, $5 \%$ dialyzed fetal bovine serum and $10 \mu \mathrm{M}$ calcium chloride. Barrier function was monitored by incubating cells in LCM for 16-24 hr. TER and inulin-flux were measured in transwells at time points ranging from 1 to $24 \mathrm{hr}$. The media was then switched with complete media containing NCM and barrier function was monitored for 2 hr. Live-cell imaging of GFP was performed in confluent monolayers in $60 \mathrm{~mm}$ dishes. 




Figure 2-2. $\quad \mathrm{Ca}^{2+}$ Switch Assay.

$\mathrm{Ca}^{2+}$ switch technique is used to study de novo assembly and disassembly of TJ.

Confluent cell monolayers are incubated in $\mathrm{Ca}^{2+}$ depleted media to induce TJ

disassembly. Then the low calcium media (LCM) is switched with normal calcium media (NCM) to induce TJ assembly. TJ integrity is monitored by measuring TER, flux and imaging. 


\section{Recombinant GST-Ocl-C Expression}

\section{Transformation}

The C-terminal region of human occludin encoding the amino acid sequence from 378-522 (Ocl-C) was amplified from the pEGFP-Occludin vector. The amplicon was then cloned into pGEX-2T vector (Amersham, Arlington Heights, IL). Recombinant Ocl-C was produced as GST fusion protein (GST-Ocl-C) by transformation of the construct in E. coli BL21 cells. Glycerol stocks were prepared and stored at $-80^{\circ} \mathrm{C}$.

\section{Culture Scale-up}

The culture from glycerol stock was streaked on agar containing ampicillin (Amp; $50 \mathrm{ug} / \mathrm{ml}$ ). The resistant colony was inoculated in $2 \mathrm{ml} \mathrm{LB}$-broth and incubated at $37^{\circ} \mathrm{C}$ and $250 \mathrm{rpm}$ for $6-8 \mathrm{hr}$. This $2 \mathrm{ml}$ culture was then transferred to $100 \mathrm{ml} \mathrm{LB}+\mathrm{Amp}$ and incubated at $37^{\circ} \mathrm{C}$ and $250 \mathrm{rpm}$ overnight. The culture was then inoculated into $400 \mathrm{ml}$ $\mathrm{LB}+\mathrm{Amp}$ and incubated at $37^{\circ} \mathrm{C}$ and $250 \mathrm{rpm}$. After $2 \mathrm{hr}, 500 \mathrm{ul}$ or $0.5 \mathrm{ml}$ of $1 \mathrm{M} \mathrm{IPTG}$ $(1 \mathrm{mM})$ was added to the $500 \mathrm{ml}$ culture and incubated for another $4.5 \mathrm{hr}$. The culture was centrifuged at $3500 \mathrm{rpm}$ for $30 \mathrm{~min}$ at $4^{\circ} \mathrm{C}$. The supernatant was discarded and the pellet was stored at $-80^{\circ} \mathrm{C}$.

\section{Protein Extraction}

The pellet was resuspended thoroughly in $15 \mathrm{ml}$ lysis buffer $(50 \mathrm{mM}$ Tris, $1 \mathrm{mM}$ EDTA, 10\% Sucrose, $\mathrm{pH} 7.3$ ) containing protease inhibitor cocktail 10ul $/ \mathrm{ml}$ each of PMSF, NaF, Na3VO4. $1.5 \mathrm{ml}$ lysozyme $(10 \mathrm{mg} / \mathrm{ml})$ was added and incubated with rotation at $4{ }^{\circ} \mathrm{C}$ for $30 \mathrm{~min} .0 .75 \mathrm{ml}$ of freshly prepared $10 \%$ NP40 (IGEPAL) detergent was added and incubated further on the rotator for another $30 \mathrm{~min}$ at $4{ }^{\circ} \mathrm{C}$. The Lysate was sonicated to a uniform suspension setting 15 with 1 -second strokes ( 30 strokes). The suspension was then centrifuged at $12000 \mathrm{rpm}(20,000 \mathrm{~g})$ for $1 \mathrm{hr}$ and supernatant was collected.

\section{Protein Purification}

$750 \mathrm{ul}$ of GSH agarose (Sigma) beads (per 15ml supernatant) was prewashed with cold PBS-IF three times by centrifuging at 13500rpm for $2 \mathrm{~min}$ at $4^{\circ} \mathrm{C}$. The beads were added to the extracted supernatant and incubated for $2 \mathrm{hr}$ on the rotator at $4^{\circ} \mathrm{C}$. After the incubation, the beads were collected by centrifuging at $13500 \mathrm{rpm}$ for $10 \mathrm{~min}$ at $4{ }^{\circ} \mathrm{C}$. The beads were washed 5 times with cold PBS. Then $1 \mathrm{ml}$ of reduced glutathione $(20 \mathrm{mM})$ was added to the beads and incubated for $5 \mathrm{~min}$ on the rotator. Supernatant was collected by centrifuging at 2000rpm for $1 \mathrm{~min}$ at $4^{\circ} \mathrm{C}$. 


\section{Dialysis}

Supernatant was injected into dialysis cassette (3500 MWCO) and immersed with a floater in dialysis buffer $(50 \mathrm{mM}$ Tris, $150 \mathrm{mM} \mathrm{NaCl}, 0.5 \mathrm{mM}$ EGTA, $0.05 \%$ beta mercaptoethanol). Buffer was changed every 2 hours, 3 times and then left overnight. Protein was estimated in the dialyzed sample.

\section{Occludin Regulatory Peptide (ORP)}

\section{ORP Characteristics}

Occludin regulatory peptide (ORP) was custom synthesized by Biomatik. ORP comprises of amino acid sequence analogous to ORM (Mol.wt 458193) with a biotin tag at its N-terminus and a TAT sequence at its C-terminus. The molecular formula of ORP

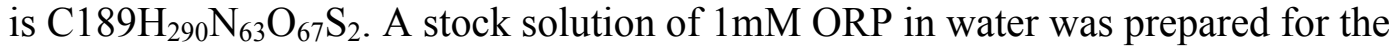
experiments. Peptide with scrambled sequence was used as control referred to as ORPscr.

\section{ORP Permeability Studies}

- Caco-2 cell monolayers were grown to confluence on transwells. Cells were treated with $3 \mu \mathrm{M}$ and $10 \mu \mathrm{M}$ ORP and incubated at $37^{\circ} \mathrm{C}$ for $30 \mathrm{~min}$. The monolayers were fixed and probed with $\mathrm{Cy} 3$ conjugated Streptavidin. Fluorescent images were collected by confocal microscopy.

- Intestinal sacs from C57BL/6 mice were prepared. One end of the intestinal segment was sutured and $10 \mu \mathrm{M}$ peptide solution containing FITC-inulin $(0.5 \mathrm{mg} / \mathrm{ml})$ was injected from the other end. This end was then sutured to form a sac. The sacs were incubated in DMEM at $37^{\circ} \mathrm{C}$ for $1 \mathrm{hr}$. Cryosections were prepared and immunostained for $\mathrm{ZO}-1$, biotin and nucleus.

\section{$\mathrm{Ca}^{2+}$ Switch Assay in Presence of ORP}

Caco-2 cells were seeded on transwells and allowed to become confluent. Cells were exposed to DMEM containing 4mM EGTA for 20 min. Following EGTA treatment media was replaced with DMEM containing $10 \mu \mathrm{M}$ ORP and FITC-inulin $(0.5 \mathrm{mg} / \mathrm{ml})$. TER and inulin flux were measured every hour up to 6 hours during recovery. Cell monolayers were fixed in acetone: methanol mixture and immunostained for occludin and $\mathrm{ZO}-1$. 


\section{Irradiation in Presence of ORP}

Confluent monolayers of Caco-2 cells in transwells were exposed to radiation (4Gy) and barrier function was compared to the sham treated control. Following radiation FITC-inulin was added to the media and flux and TER were measured every hour up to 5 hr. Next, the cell monolayers were pre-incubated with $10 \mu \mathrm{M}$ ORP for $30 \mathrm{~min}$ and then exposed to radiation. Following radiation, FITC-inulin was added and barrier function was assessed by measuring TER and inulin flux. The cell monolayers were fixed and immunostained for occludin, ZO-1 and nucleus.

\section{ORP Pull Down Assay}

\section{ORP Phosphorylation}

- ATP working solution was prepared in Mg buffer containing 20mM MOPS, $25 \mathrm{mM} \beta$-glycerophosphate, $750 \mathrm{mM} \mathrm{MgCl} 2,5 \mathrm{mM}$ EDTA, $1 \mathrm{mM}$ vanadate and $1 \mathrm{mM}$ DTT.

- To induce tyrosine phosphorylation, ORP $(1 \mathrm{mM})$ was incubated with 0.5 ug active $\mathrm{c}$-Src in tyrosine kinase buffer (50 mm Hepes, $\mathrm{pH} 7.4,1 \mathrm{~mm}$ EDTA, $0.2 \% \beta$ mercaptoethanol, $3 \mathrm{~mm} \mathrm{MgCl} 2$ ) containing $100 \mu \mathrm{m}$ ATP at $30^{\circ} \mathrm{C}$ for $3 \mathrm{hr}$ on a thermo mixer. Control reactions were performed in the absence of ATP.

- To induce threonine phosphorylation, ORP $(1 \mathrm{mM})$ was incubated with $0.5 \mathrm{ug}$ casein kinase-2 (CK-2) in threonine kinase buffer ( $80 \mathrm{mM}$ MOPS/NaOH $(\mathrm{pH} 7.0)$ containing $2 \mathrm{mM}$ EDTA) containing $100 \mu \mathrm{m} \mathrm{ATP}$ at $30^{\circ} \mathrm{C}$ for $3 \mathrm{hr}$ on a thermo mixer. Control reactions were performed in the absence of ATP.

- ORP was trypsin digested to remove TAT sequence. Trypsin stock solution of $200 \mu \mathrm{g} / \mathrm{ml}$ was prepared by dissolving $20 \mu \mathrm{g}$ of lyophilized trypsin in $100 \mu \mathrm{l}$ of resuspension buffer (50 $\mathrm{mM}$ acetic acid). Trypsin stock was diluted 1 in 5 in $112.5 \mathrm{mM} \mathrm{NH}_{4} \mathrm{HCO}_{3}$ to prepare the working solution. This working solution was then incubated for $15 \mathrm{~min}$ at $37^{\circ} \mathrm{C}$. ORP was incubated with equal volume of working trypsin solution for 3 hours at $37^{\circ} \mathrm{C}$ and mixed with TPCK solution after incubation. This ORP was now used as non-phosphorylated control.

\section{ORP Pull Down}

Caco-2 cells in T75 flask were grown to confluence. Cells were washed in icecold PBS and extracted in lysis buffer $(0.2 \%$ Triton X-100 in PBS containing $10 \mathrm{mM}$ sodium fluoride, $1 \mathrm{mM}$ sodium pyrophosphate, $1 \mathrm{mM}$ sodium ortho vanadate, $1 \mathrm{mM}$ PMSF and protease inhibitor cocktail $(10 \mu \mathrm{l} / \mathrm{ml}) .1 \mathrm{ml}$ of extract was added to each of the non-phosphorylated, tyrosine phosphorylated and threonine phosphorylated ORP 
triplicates. These mixtures were then incubated overnight at $4^{\circ} \mathrm{C}$ on rocker. $50 \mu \mathrm{l}$ of streptavidin-Agarose (prewashed in cold PBS) was added to each sample and incubated for 1 hour at $4^{\circ} \mathrm{C}$ on rocker. The beads were centrifuged, washed and subjected to LCMS/MS analysis.

\section{LC-MS/MS Analysis}

\section{Sample Workup}

Sample bead pellets (3) were suspended in $100 \mu \mathrm{Tris}-\mathrm{HCl}(500 \mathrm{mM}, \mathrm{pH} 8.5)$, $\mathrm{CaCl}_{2}(100 \mathrm{mM})$. The samples were centrifuged for 2 minutes at $10,000 \mathrm{xg}$ and the supernatant was recovered. This process was repeated one more time. The combined supernatant was treated with $5 \mu$ of the reducing reagent dithiothreitol (DTT, $1 \mathrm{M}$ in $\mathrm{dH} 2 \mathrm{O}$ ) and incubated at $37{ }^{\circ} \mathrm{C}$ for $30 \mathrm{~min}$. The reduced samples were incubated with $5 \mu 1$ of the cysteine alkylating reagent iodoacetamide (IAA, $2.2 \mathrm{M}^{\text {in } \mathrm{dH}_{2} \mathrm{O} \text { ) for } 15 \mathrm{~min} \text { at }}$ room temperature, in the dark. After alkylation, a second aliquot of DTT was added $(5 \mu$, $1 \mathrm{M}$ in $\mathrm{dH}_{2} \mathrm{O}$ ) to neutralize excess IAA.

Following reduction and alkylation, $5 \mu \mathrm{l}$ of sequencing grade trypsin $(1 \mu \mathrm{g} / \mu \mathrm{l})$ were added and the samples were digested at $37^{\circ} \mathrm{C}$ overnight. After digestion each sample volume was reduced by speed vacuum centrifugation to about $20 \mu 1$. Trypsin was neutralized by addition of $3 \mu \mathrm{l}$ of neat trifluoro acetic acid (TFA). The peptides were purified with ZipTip C18 (Millipore) using procedures recommended by the manufacturer. The peptides bound to the C18 column were eluted with $4 \mu \mathrm{L}$ of $50 \%$ acetonitrile $/ 50 \%$ water $/ 0.1 \%$ TFA. Two ZipTips per sample were used, and elution was performed with the same $4 \mu \mathrm{L}$-aliquot. After purification, the samples were diluted with 8 $\mu \mathrm{L}$ of water $/ 0.5 \%$ formic acid.

\section{LC-MS/MS}

The LC-MS/MS analyses were performed with an LTQ linear ion trap mass spectrometer (Thermo Scientific) interfaced with a Famos/Ultimate nanoflow LC system (Dionex). Two microliters of peptide digests were manually injected onto a nano injector. Peptides were separated on a home-packed capillary column $(100 \mathrm{~mm} \times 75 \mu \mathrm{m}$ I.D. $)$ packed with reversed phase $\mathrm{C} 18$ silica $(5 \mu \mathrm{m}, 200 \AA)$. The peptides were eluted at a flow rate of $300 \mathrm{~nL} / \mathrm{min}$ with a 95 min linear gradient from solvent $\mathrm{A}$ $\left[\left(\mathrm{H}_{2} \mathrm{O}: \mathrm{CH}_{3} \mathrm{OH}: \mathrm{HCOOH}\right),(97.9: 2: 0.1)\right.$, v:v:v] to solvent $\mathrm{B}\left[\left(\mathrm{H}_{2} \mathrm{O}: \mathrm{CH}_{3} \mathrm{OH}: \mathrm{HCOOH}\right)\right.$, (9.9:90:0.1), v:v:v]. The mass spectrometer was operated in a data-dependent scan mode, where a survey MS scan $(400-2000 \mathrm{~m} / \mathrm{z})$ was followed by MS/MS of the seven most intense precursor ions with dynamic exclusion on. 


\section{Peptide Data Analysis}

The raw data were input into Proteome Discoverer version 1.4 and searched with Sequest HT against a human database in silico digested with trypsin. The search parameters were: full tryptic specificity, precursor error tolerance $1.5 \mathrm{Da}$, product ion tolerance $0.8 \mathrm{Da}$. Allowed peptide modifications were carbamidomethylation of cysteine (static modification), and methionine oxidation and lysine acetylation (dynamic modifications). To calculate the false discovery rate for matched peptides, the same rat reversed database was used as a decoy and the resulting data were statistically analyzed by Percolator, a new standard component of Proteome Discoverer. Only peptides with PEP scores of 0.01 or lower were considered as positive matches.

\section{Protein Differential Expression Analysis}

The data from the Proteome Discover search results were further analyzed with the Scaffold software. Scaffold performs advanced processing of MS/MS data, spectral counting, and comprehensive data management. Scaffold uses statistical algorithms to validate search results and accepts peptide and protein identifications established at $\geq 95 \%$ probability. T-test statistics was used to identify differentially expressed proteins $(\mathrm{p} \leq$ $0.05)$.

\section{ORP and GST-Ocl-C Pull Down for MAP7}

\section{ORP/ORPscr Tyrosine Phosphorylation}

ORP and ORPscr $(0.5 \mathrm{mM})$ were incubated with 0.5 ug active c-Src in tyrosine kinase buffer (50 mm HEPES, pH 7.4, $1 \mathrm{~mm}$ EDTA, $0.2 \% \beta$-mercaptoethanol, $3 \mathrm{~mm}$ $\mathrm{MgCl}_{2}$ ) containing $100 \mu \mathrm{m} \mathrm{ATP}$ at $30^{\circ} \mathrm{C}$ for $3 \mathrm{hr}$ on a shaking incubator. These samples were referred to as $\mathrm{pY}$ (tyrosine phosphorylated) forms of ORP and ORPscr. Samples from similar reactions performed in the absence of ATP were referred to as NP (nonphosphorylated) forms of ORP and ORPscr.

\section{GST-Ocl-C/GST Tyrosine Phosphorylation}

Recombinant GST-Ocl-C (20 $\mu \mathrm{g})$ and equimolar amount of GST $(\sim 10 \mu \mathrm{g})$ were incubated with $0.5 \mathrm{ug}$ active c-Src in tyrosine kinase buffer $(50 \mathrm{~mm}$ Hepes, $\mathrm{pH} 7.4,1 \mathrm{~mm}$ EDTA, $0.2 \% \beta$-mercaptoethanol, $3 \mathrm{~mm} \mathrm{MgCl} 2$ ) containing $100 \mu \mathrm{m}$ ATP at $30^{\circ} \mathrm{C}$ for $3 \mathrm{hr}$ on a shaking incubator. These samples were referred to as $\mathrm{pY}$ (tyrosine phosphorylated) forms of GST-Ocl-C and GST. Samples from similar reactions performed in the absence of ATP were referred to as NP (non-phosphorylated) forms of GST-Ocl-C and GST. 


\section{Pull Down}

Caco-2 cell extracts were prepared in lysis buffer $(0.2 \%$ Triton X-100 in PBS containing $10 \mathrm{mM}$ sodium fluoride, $1 \mathrm{mM}$ sodium pyrophosphate, $1 \mathrm{mM}$ sodium ortho vanadate, $1 \mathrm{mM}$ PMSF and protease inhibitor cocktail $(10 \mu \mathrm{l} / \mathrm{ml})$. ORP-NP, ORP-pY, ORPscr-NP, ORPscr-pY, GST-Ocl-C-NP, GST-Ocl-C-pY, GST-NP, GST-pY were incubated with $1 \mathrm{ml}$ of cell extract overnight at $4{ }^{\circ} \mathrm{C}$ on a $\mathrm{n}$ inverter. $30 \mu \mathrm{l}$ of pre-washed streptavidin agarose or GSH-agarose beads were added to each sample and incubated at 4 ${ }^{\circ} \mathrm{C}$ for $1 \mathrm{hr}$. The beads were collected by centrifugation, washed and mixed with sample buffer. The beads were heated at $100^{\circ} \mathrm{C}$ for $10 \mathrm{~min}$ and supernatant was collected by centrifugation. The samples were run on $7 \%$ tris-acetate gel and immunoblotted for MAP-7.

\section{Co-immunoprecipitation: Occludin/MAP7}

Caco-2 cell monolayers in $60 \mathrm{~mm}$ culture dishes were treated with $3 \%$ DSS or 0.35M mannitol in DMEM for $3 \mathrm{hr}$. The cells were washed with ice-cold PBS and proteins were extracted in the IP buffer (50 mM Tris buffer, $\mathrm{pH} 7.4$, containing 1\% NP40, 2 mM EDTA, $2 \mathrm{mM}$ EGTA, $10 \mathrm{mM}$ sodium fluoride, $1 \mathrm{mM}$ vanadate and protease inhibitor cocktail $(10 \mu \mathrm{g} / \mathrm{ml}))$. Protein concentration was estimated by BCA method. $1 \mathrm{mg}$ protein lysates were incubated with $2 \mu \mathrm{g}$ monoclonal anti-occludin antibody at $4{ }^{\circ} \mathrm{C}$ overnight on an inverter. Immune complexes were collected by precipitation using protein-A/G plus agarose beads and incubation at $4^{\circ} \mathrm{C}$ for $1 \mathrm{hr}$. The beads were pelleted, washed, resuspended in sample buffer and heated at $100^{\circ} \mathrm{C}$ for $10 \mathrm{~min}$ to extract the bound protein. Samples were run on $7 \%$ tris acetate gel and immunoblotted for MAP-7.

\section{Colocalization Analysis: Occludin/MAP7}

Animal studies were performed according to an IACUC approved protocol. C57BL/6 mice (12 weeks old) were subjected to radiation (4 Gy). $2 \mathrm{hr}$ post radiation, colon was harvested from the mice. Cryosections of the colon were prepared and fixed in acetone:methanol mixture. The fixed tissue sections were immunostained for occludin and MAP-7. Images were collected by confocal microscopy.

\section{Sources of Materials}

\section{Antibodies}

Rabbit polyclonal anti-ZO-1, mouse monoclonal anti-occludin \& anti-claudin 2, Alexa Fluor 488-conjugated anti-mouse IgG \& phalloidin were purchased from Invitrogen (Carlsbad, CA). Mouse monoclonal anti-GFP antibody was purchased from Clontech (Mountain view, CA). Hoechst 33342 were obtained from Life technologies 
(Carlsbad, CA). Mouse monoclonal anti-E-Cadherin and rabbit polyclonal anti-b-catenin were purchased from BD Biosciences (San Jose, CA). Rabbit polyclonal anti-MAP7, anti-plakophilin-3, rabbit monoclonal anti-peroxiredoxin 1 and mouse monoclonal antiHSP60 were obtained from EMD Millipore (Bedford, MA). Cy3-conjugated anti-rabbit IgG, HRP-conjugated anti-mouse IgG, HRP-conjugated anti-rabbit IgG, and mouse anti$\beta$-actin antibodies were obtained from Sigma (St Louis, MO). GFP-Trap_A was purchased from Chromotek (Hauppauge, NY). Restriction enzymes, T4 DNA ligase, polynucleotide kinase and DPn1 were obtained from New England Biolabs (Beverly, MA).

\section{Chemicals}

Dulbecco's minimum essential media (DMEM), fetal bovine serum (FBS) and antibiotics were purchased from Cellgro ${ }^{\circledR}$ (Manassas, VA) while Calcium free DMEM was from Thermo Fisher Scientific (Tustin, CA.). Cytochalasin-D and genistein were obtained from Sigma Aldrich (St. Louis, MO). FITC-inulin, protease inhibitor cocktail, phenylmethylsulfonyl fluoride, Triton-X-100, streptavidin-agarose, GSH-agarose and IPTG were purchased from Sigma (St. Louis, MO). Protein A/G plus beads were obtained from Santa Cruz (Dallas, TX). All other chemicals were purchased from Sigma or Fisher.

\section{Statistical Analysis}

All data are expressed as Mean \pm SEM. The differences among multiple groups were first analyzed by ANOVA. When a statistical significance was detected, Tukey's t test was used to determine the statistical significance between multiple testing groups and the corresponding control. Statistical significance was established at $95 \%$.

Data containing confocal images was collected from experiments repeated at least three times. Images were collected from at least three different locations of a stained monolayer and the best representative image was selected for the figures. Fluorescence intensity at the intercellular junctions was quantitated using Image $\mathrm{J}$ by selecting 20 random ROIs from one image. Statistical analysis was performed by using ANOVA. 


\section{CHAPTER 3. RESULTS}

\section{Specific Aim 1: To Determine That ORM Confers Dynamics to TJ in a Phosphorylation-dependent Mechanism}

\section{Generation of Ocl ${ }^{\mathrm{DM}}$}

It is evident from a significant body of literature that ORM is an active site for phosphorylation. In order to determine the functional significance of ORM and occludin in TJ dynamics and regulation, a deletion mutant of occludin that lacks ORM was generated in a pEGFP plasmid containing human occludin gene (Figure 3-1A). Restriction digestion, PCR amplification and DNA sequencing confirmed the deletion of ORM (Figure 3-1B).

Although ORM corresponds to 11 amino acids (Y398-S408), a larger deletion was designed to include the highly conserved region on either side of ORM (Figure 31C). This resulted in a truncated occludin $\left(\mathrm{Ocl}^{\mathrm{DM}}\right)$, which lacks 28 amino acids (G388D415) compared to $\mathrm{Ocl}^{\mathrm{WT}}$ (Figure 3-1D). The ultimate goal was to perform truncation analysis of ORM to identify the residues that are essential enough to confer dynamic properties to TJ and occludin itself.

\section{Deletion of ORM Does Not Prevent Occludin Assembly at the TJ}

C-terminus of occludin is known to be required for the assembly and function of TJ barrier (82). First we examined whether ORM is required for occludin localization at TJ. To avoid potential interactions with endogenous occludin, occludin-deficient MDCK (OD-MDCK) cells were used. Stable clones expressing GFP-Ocl ${ }^{\mathrm{WT}}, \mathrm{GFP}^{-O l^{\mathrm{DM}}}{ }^{\text {or }}$ vector were generated and live imaging was performed to visualize GFP. At least 12 clones were screened for fluorescence, of which 6 are reported here.

$\mathrm{In} \mathrm{Ocl}^{\mathrm{DM}}$ cells, GFP-occludin was localized at the $\mathrm{TJ}$ similar to that in $\mathrm{Ocl}^{\mathrm{WT}}$ cells, where as GFP was intracellular in vector-transfected cells (Figure 3-2A). There was no clonal difference as occludin localization was similar in all the clones screened (Figure 3-2B). This suggested that ORM is not required for occludin assembly at TJ.

\section{Absence of ORM in Occludin Does Not Prevent Its Interaction with ZO-1}

Occludin C-terminus comprises of a coiled-coil domain that interacts with ZO-1 and this interaction is believed to be required for localization of occludin at TJ (18). First the expression of mutant occludin versus wild type was examined. Total protein was immunoblotted for occludin and GFP. Then to look at occludin/ZO-1 interaction, GFP was immunoprecipitated and immune complexes were blotted for ZO-1 and GFP. 

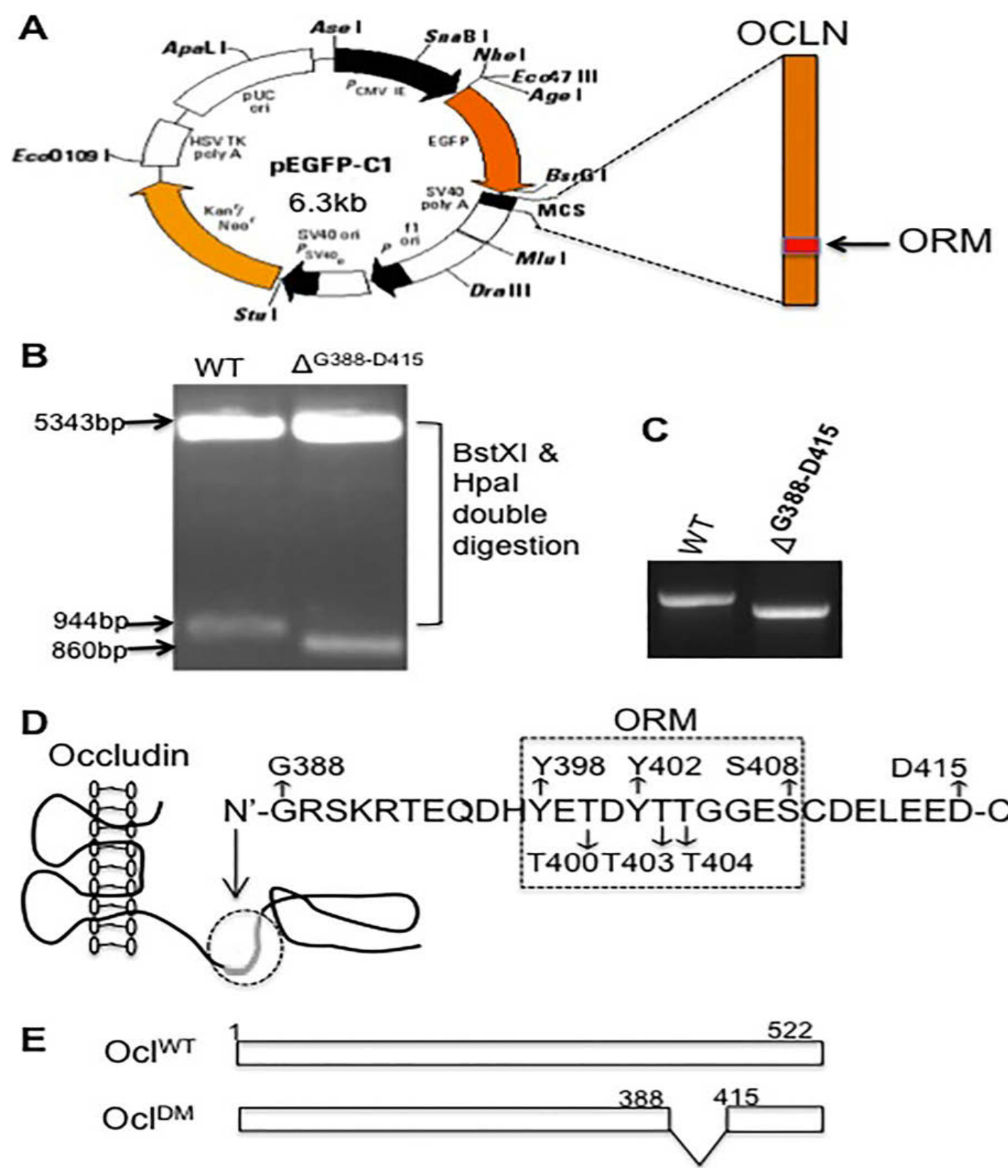

Figure 3-1. Occludin Deletion Mutant That Lacks ORM (Ocl $\left.{ }^{\mathrm{DM}}\right)$.

A. pEGFP construct comprising of human occludin gene. ORM is located in the distal part of the sequence (Red). B. Double digestion of wild type and mutant constructs with BstXI and HpaI resulted in two fragments. The smaller fragment in case of mutant construct had a molecular weight lower than that in wild type construct confirming deletion of ORM. C. PCR amplification of the nucleotide sequence including ORM further confirmed the deletion of ORM. D. Schematic showing the location of ORM in occludin and the amino acid sequence of ORM. E. ORM deletion resulted in $\mathrm{Ocl}^{\mathrm{DM}}$ lacking the amino acid sequence from 388 to 415 . 

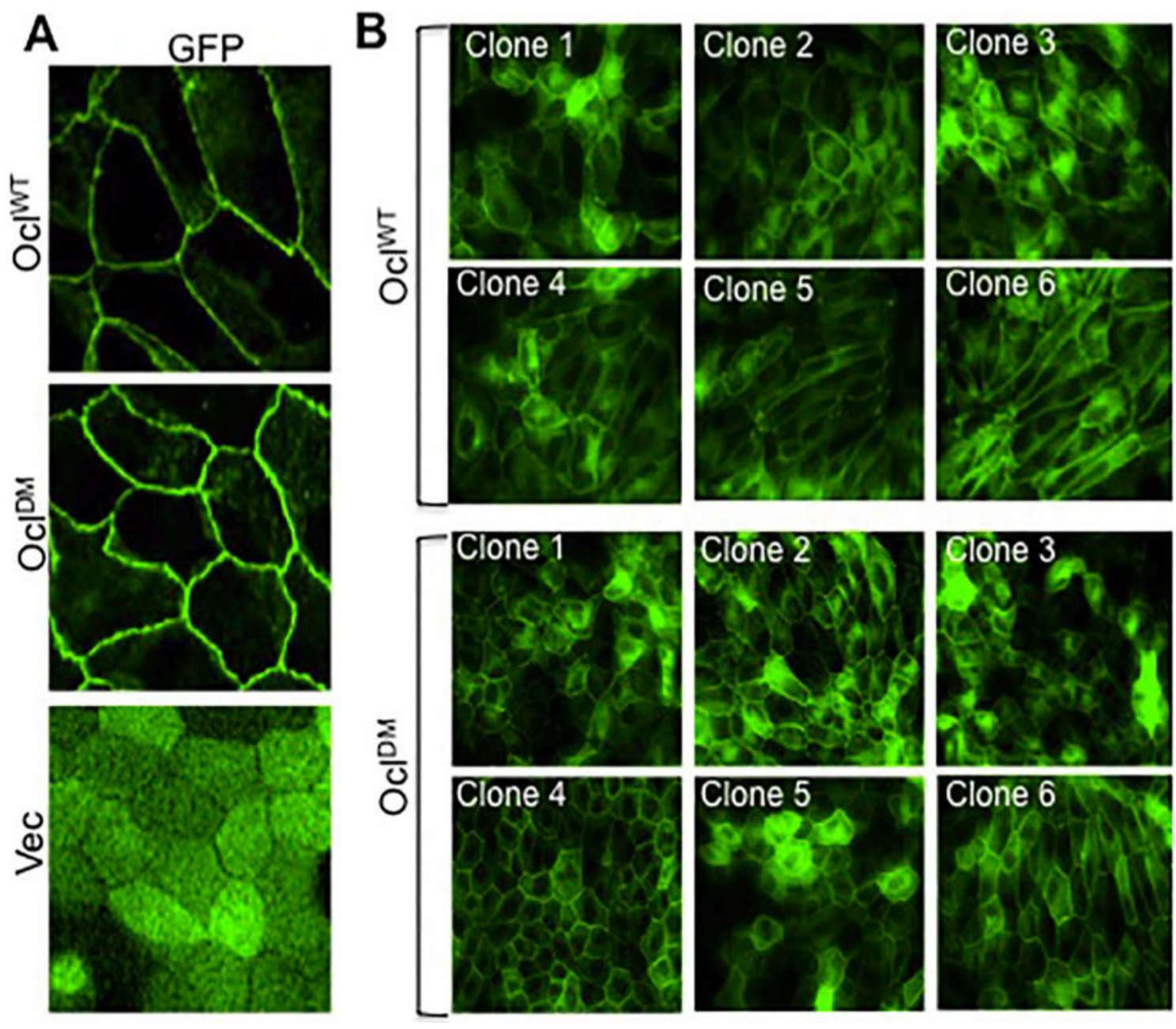

Figure 3-2. ORM Deletion Does Not Prevent Assembly of Occludin into TJ.

A: $\mathrm{Ocl}^{\mathrm{WT}}, \mathrm{Ocl}^{\mathrm{DM}}$ and Vec cell monolayers were imaged live for GFP fluorescence by confocal microscopy. B: Six clones of $\mathrm{Ocl}^{\mathrm{WT}}$ and $\mathrm{Ocl}^{\mathrm{DM}}$ cell monolayers were imaged live for GFP fluorescence using a 40X objective by epi-fluorescence microscopy. 
Immunoblot for occludin showed the knockdown of endogenous occludin in all the clones. Immunoblot for GFP showed that GFP-Ocl ${ }^{\mathrm{DM}}$ has a molecular weight lower than GFP-Ocl $^{\text {WT }}$, confirming the deletion (Figure 3-3A). Immunoprecipitation of GFP showed that GFP-Ocl ${ }^{\mathrm{DM}}$ interacts with occludin similar to wild type (Figure 3-3B) and the densitometry confirmed no significant difference in the amount of occludin pulled down with GFP (Figure 3-3C). This suggested that ORM is not required for interaction between occludin and ZO-1.

\section{Lack of Both ORM and Occludin Decreases Steady-state Resistance but Does Not Affect Leak Pathway Permeability}

To assess the effect of ORM deletion on barrier function, post-seeding barrier development was monitored by seeding cells on transwells. TER and unidirectional flux of FITC-inulin was measured everyday up to 4 days.

Vector transfected cells developed low resistance on day-1, which did not change even after 4 days post-seeding. Re-expression of wild type occludin ( $\mathrm{Ocl}^{\mathrm{WT}}$ ) in OD cells increased steady state resistance from day-1 to day-4. However, the expression of mutant occludin showed no increase in TER (Figure 3-4A). On the other hand, although there was an initial difference in inulin-flux on day- $1 \& 2$, inulin permeability was similar among these cells by day-4 (Figure 3-4B). This significant difference in TER with no apparent change in inulin flux that represents leak pathway, suggested that the permeability of ions or solutes smaller than inulin was altered in the absence of either ORM or occludin itself.

\section{Lack of ORM or Occludin Enhances Claudin-2 Expression at the TJ}

To explain the differences in TER, we looked at the expression and distribution of claudin-2 which is a major cationic pore forming claudin (25). Confluent cell monolayers were fixed in acetone/methanol mixture and immunostained for claudin-2. Total protein extracts from the cells were immunoblotted for claudin- 2 .

A significant increase in claudin-2 localization at TJ was seen in cells expressing $\mathrm{Ocl}^{\mathrm{DM}}$ compared to that in $\mathrm{Ocl}^{\mathrm{WT}}$ cells (Figure 3-5A). This increase in claudin-2 was much more prominent in the absence of occludin as confirmed by a measure of claudin-2 fluorescence intensity at the TJ (Figure 3-5B). The immuoblot for claudin-2 showed an increase in claudin-2 expression in the absence of both ORM and occludin compared to wild type (Figure 3-5C) that was confirmed by densitometry (Figure 3-5D). These data support the observation that occludin plays a role in pore pathway permeability by regulating claudin-2 expression. 


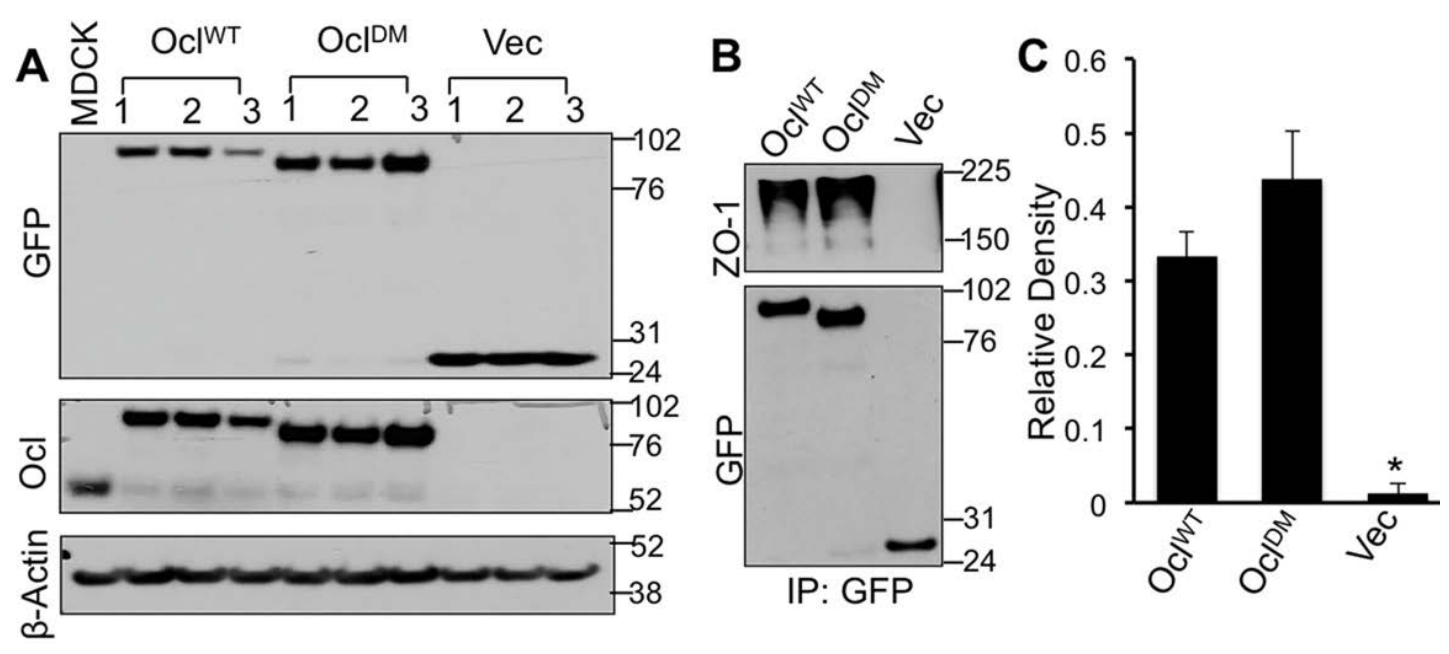

Figure 3-3. ORM Deletion in Occludin Does Not Prevent Interaction with ZO-1.

A: Total protein extracts from $\mathrm{Ocl}^{\mathrm{WT}}, \mathrm{Ocl}^{\mathrm{DM}}$ and Vec cells were immunoblotted for GFP, occludin and $\beta$-actin. B: GFP was immunoprecipitated from the native extracts of $\mathrm{Ocl}^{\mathrm{WT}}$, $\mathrm{Ocl}^{\mathrm{DM}}$ and Vec cells and immunoblotted for ZO-1. C: Density of ZO-1 was measured and normalized to corresponding GFP band density. Values presented in the graph are mean \pm $\operatorname{sem}(n=3)$. Asterisk indicates that the value is significantly $(p<0.05)$ different from corresponding $\mathrm{Ocl}^{\mathrm{WT}}$ value.
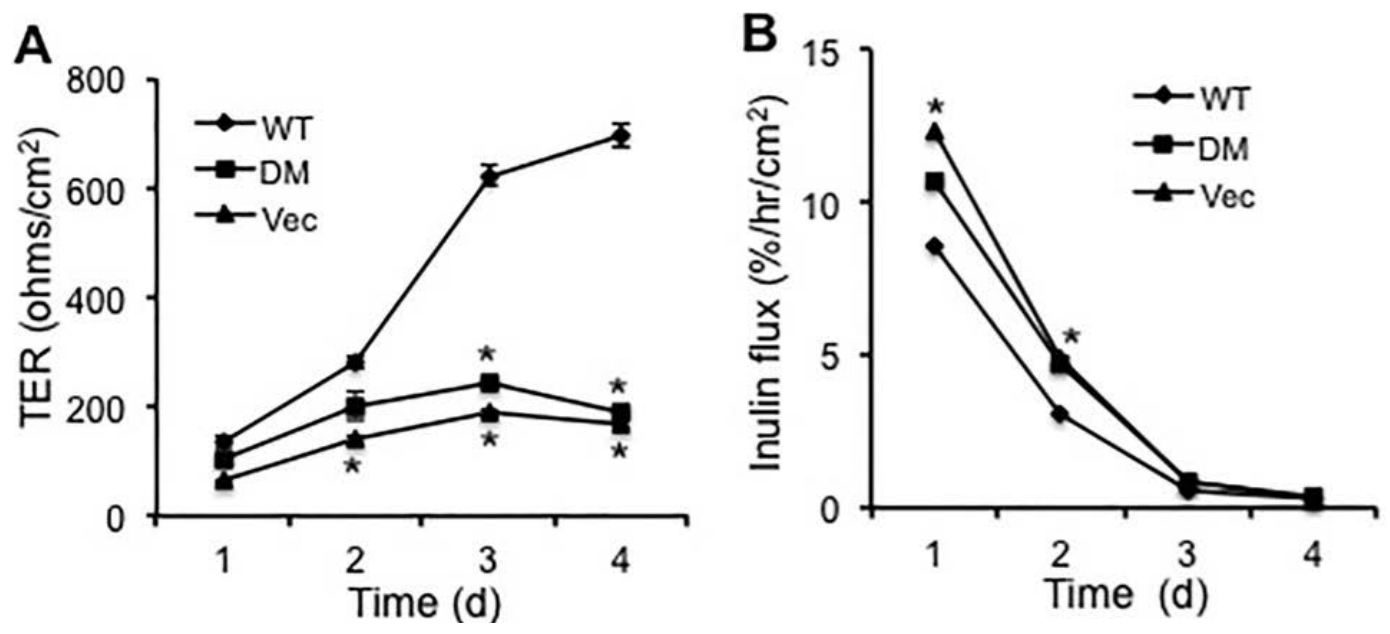

Figure 3-4. ORM Deletion Decreases Steady-state Resistance of the Barrier.

A \& B: Equal number of Ocl ${ }^{\mathrm{WT}}, \mathrm{Ocl}^{\mathrm{DM}}$ and Vec cells were seeded onto transwell inserts. TER (A) and FITC-inulin flux (B) were measured at varying post-seeding times. Values presented in the graph are mean $\pm \operatorname{sem}(n=6)$. Asterisks indicate the values that are significantly $(\mathrm{p}<0.05)$ different from corresponding values for $\mathrm{Ocl}^{\mathrm{WT}}$ group. 

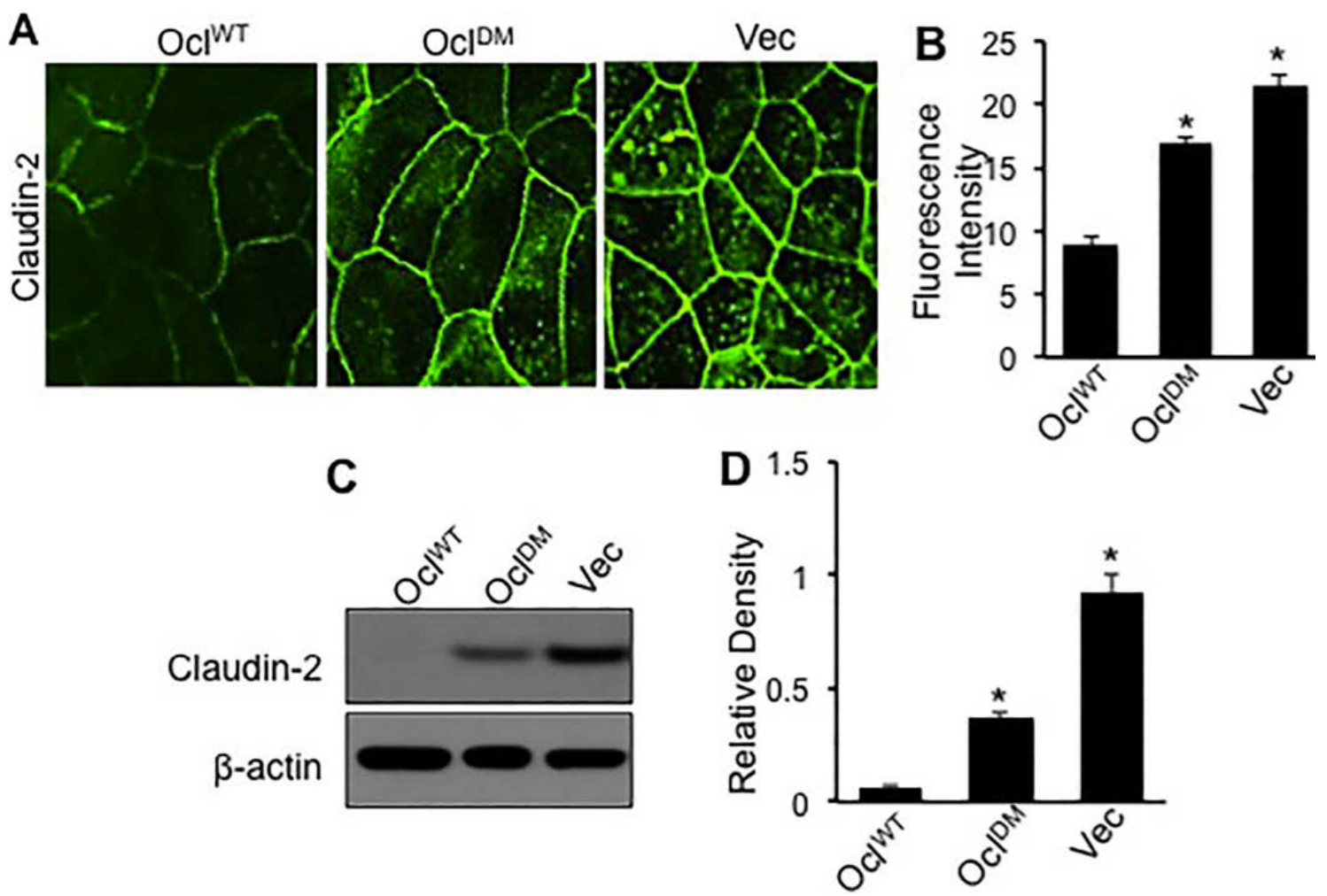

Figure 3-5. Absence of Occludin or ORM Enhances Claudin-2 Localization at TJ.

A \& B: Fixed cell monolayers of Ocl ${ }^{\mathrm{WT}}, \mathrm{Ocl}^{\mathrm{DM}}$ and $\mathrm{Vec}$ were immunostained for claudin-2 (green) (A) and fluorescence intensity at intercellular junctions was quantitated (B). C \& D: Protein extracts were immunoblotted for claudin-2 and $\beta$-actin (C). Density of claudin-2 was measured and normalized to $\beta$-actin band density (D). Values presented in the graph are mean $\pm \operatorname{sem}(n=3)$. Asterisk indicates that the value is significantly $(\mathrm{p}<0.05)$ different from corresponding $\mathrm{Ocl}^{\mathrm{WT}}$ value. 


\section{Lack of Both ORM and Occludin Enhances Cytoskeletal Organization and Occludin Localization at $\mathbf{T J}$}

A non-ionic detergent based protein extraction is widely used to examine the shift of TJ components between the insoluble and soluble fractions during TJ assembly or disassembly. Most of the TJ components are pulled down along with the actin cytoskeleton-rich insoluble fraction. Since the mutant occludin appeared to be distinctly localized at TJ, we investigated whether there was any difference in the amount of occludin localization at TJ.

The cells monolayers from day 1 and 3 post-seeding were fixed in 3\% paraformaldehyde and double-labeled for actin and tubulin. Lysates from confluent cell monolayers were subjected to triton X-100 based lysis and centrifuged to isolate supernatant, which is referred to as Triton-soluble fraction. The pellet was resuspended and sonicated to obtain actin -rich fraction referred to as Triton-insoluble fraction. The lysates were immunoblotted for GFP and actin.

Confocal microscopy showed that actin cytoskeleton was better organized at the intercellular junctions of $\mathrm{Ocl}^{\mathrm{DM}}$ and Vec cell monolayers compared to that in $\mathrm{Ocl}^{\mathrm{WT}}$ cell monolayers on day1. By day 3, cytoskeleton in $\mathrm{Ocl}^{\mathrm{WT}}$ appeared to have become well organized (Figure 3-6A). This suggested that the absence of either ORM or occludin enhanced cytoskeletal arrangement during barrier development. Immunoblot analysis of Triton-insoluble and soluble fractions indicated that both GFP- Ocl ${ }^{\text {WT }}$ and GFP- Ocl ${ }^{\mathrm{DM}}$ were distributed in both fractions. In the triton-soluble fraction there was no significant difference in the amount of occludin in $\mathrm{Ocl}^{\mathrm{WT}}$ versus $\mathrm{Ocl}^{\mathrm{DM}}$ cells. However, in the tritoninsoluble fraction, the amount of occludin in $\mathrm{Ocl}^{\mathrm{DM}}$ cells was significantly higher compared to that in $\mathrm{Ocl}^{\mathrm{WT}}$ cells. Vector transfected cells that lack occludin acted as a negative control (Figure 3-6B). The densitometric analysis confirmed that GFP- Ocl ${ }^{\mathrm{DM}}$ association with the Triton-insoluble fraction was significantly higher that that of GFP$\mathrm{Ocl}^{\mathrm{WT}}$ (Figure 3-6C). This indicated that absence of ORM enhanced occludin association with actin rich fraction of cells.

\section{Deletion of ORM Reduces Occludin Mobility at the Intercellular Junctions}

Because occludin localization at TJ was enhanced in ORM deletion mutant cells, we examined whether there was any difference in occludin mobility in the absence of ORM. Cells were grown to confluence and FRAP analysis was performed on day 3 . Selected regions of interest (ROI) were defined at the intercellular junctions, bleached with laser at maximum power at $488 \mathrm{~nm}$. The module was set to capture images before and after the bleach. Fluorescence recovery was quantitated from the time series images and mobile fraction was calculated.

The time series images showed that the GFP-occludin fluorescence in $\mathrm{Ocl}^{\mathrm{WT}}$ cells recovered faster than that in $\mathrm{Ocl}^{\mathrm{DM}}$ cells (Figure 3-7A). Percent recovery fluorescence of $\mathrm{Ocl}^{\mathrm{DM}}$ was significantly lower than that of $\mathrm{Ocl}^{\mathrm{WT}}$ (Figure 3-7B). The mobile fraction of 



Figure 3-6. Deletion of ORM Enhances Association of Occludin with Actin-rich Fraction of Cells.

A: Two days after seeding $\mathrm{Ocl}^{\mathrm{WT}}$, Ocl ${ }^{\mathrm{DM}}$ and Vec cells were fixed and stained for F-actin (Green), $\beta$-tubulin (MT) (Red) and nucleus (Blue). B \& C: Triton-soluble and insoluble fractions prepared from $\mathrm{Ocl}^{\mathrm{WT}}, \mathrm{Ocl}^{\mathrm{DM}}$ and $\mathrm{Vec}$ cells were immunoblotted for GFP and $\beta$ actin (B). GFP band densities were measured and normalized to corresponding $\beta$-actin band densities $(\mathbf{C})$. Values are mean $\pm \operatorname{sem}(n=3)$. Asterisks indicate the values that are significantly $(\mathrm{P}<0.05)$ different from corresponding value for $\mathrm{Ocl}^{\mathrm{WT}}$ cells. 




Figure 3-7. ORM Deletion Reduced Mobile Fraction of Occludin.

A \& B: FRAP analysis of GFP in $\mathrm{Ocl}^{\mathrm{WT}}$ and $\mathrm{Ocl}^{\mathrm{DM}}$ cell monolayers. Time-lapsed images of ROI (region of interest) at intercellular junctions were collected before and after photo bleaching (A). Fluorescence intensity in the ROI was measured and plotted against time (B). Asterisks indicate the values that are significantly $(\mathrm{P}<0.05)$ different from corresponding value for $\mathrm{Ocl}^{\mathrm{WT}}$ cells. Percent mobile fractions of $\mathrm{Ocl}^{\mathrm{WT}} \& \mathrm{Ocl}^{\mathrm{DM}}$ were calculated $(\mathbf{C})$. Values are mean $\pm \operatorname{sem}(n=8)$. Asterisk indicates the value that is significantly $(\mathrm{P}<0.05)$ different from corresponding value for $\mathrm{Ocl}^{\mathrm{WT}}$ cells. 
deletion mutant occludin significantly reduced compared to the wild type (Figure 3-7C). This indicated that ORM is required for the mobility of occludin and that its ability to exchange with the non-tight junction pools is reduced in the absence of ORM.

\section{ORM Deletion Confers Resistance to $\mathrm{Ca}^{2+}$ Depletion-mediated Occludin Disassembly}

In order to investigate the effect of ORM deletion on TJ dynamics, we performed $\mathrm{Ca}^{2+}$ switch assay in wild type and deletion mutant clones to look at the differences in TJ disassembly/ assembly. Confluent monolayers of clones expressing GFP-Ocl ${ }^{\mathrm{WT}}$ and ${\mathrm{GFP}-O c l^{\mathrm{DM}}}$ were incubated in low calcium media (LCM) for $16 \mathrm{hr}$. Live images for GFP were captured before and after the incubation, by confocal microscopy.

$\mathrm{Ocl}^{\mathrm{WT}}$ cells showed disassembly of wild type occludin from TJ as expected during $\mathrm{Ca}^{2+}$ depletion. Interestingly, deletion mutant occludin was not redistributed from TJ in $\mathrm{Ocl}^{\mathrm{DM}}$ cells (Figure 3-8). This resistance was evident in other clones as well, suggesting that ORM is required for mediating $\mathrm{Ca}^{2+}$ depletion-mediated occludin disassembly from TJ.

\section{ORM Deletion Confers Resistance to $\mathrm{Ca}^{2+}$ Depletion-mediated Barrier Dysfunction}

$\mathrm{Ca}^{2+}$ switch assay in cell monolayers grown on transwells is an important tool to understand barrier function during de novo TJ assembly and disassembly. Extracellular $\mathrm{Ca}^{2+}$ depletion induces TJ disruption, decrease in resistance and increase in permeability while $\mathrm{Ca}^{2+}$ restoration induces $\mathrm{TJ}$ assembly, increase in resistance and decrease in permeability. Here we assessed the effect of ORM deletion on barrier function during $\mathrm{Ca}^{2+}$ switch. Basal TER and inulin flux were recorded in cell monolayers in regular media (NCM). Cells were then incubated in LCM for $16 \mathrm{hr}$ and resistance and flux of inulin was recorded. LCM was switched with NCM and barrier formation was monitored by measuring TER and inulin flux.

In Ocl ${ }^{\mathrm{WT}}$ cells, $\mathrm{Ca}^{2+}$ depletion-induced a decrease in TER and increase in inulin permeability, which returned to basal levels after $\mathrm{Ca}^{2+}$ restoration. However, in $\mathrm{Ocl}^{\mathrm{DM}}$ cells that lack ORM, and in Vec cells that lack occludin, there was no significant difference in TER (Figure 3-9A) and inulin flux (Figure 3-9B) after $\mathrm{Ca}^{2+}$ depletion compared to their basal values. This further confirmed that ORM is required for $\mathrm{Ca}^{2+}$ depletion-mediated barrier dysfunction. 



Figure 3-8. ORM Deletion Attenuates $\mathrm{Ca}^{2+}$ Depletion-mediated Occudin Disassembly.

Cell monolayers of Ocl ${ }^{\mathrm{WT}}$ and $\mathrm{Ocl}^{\mathrm{DM}}$ clones were incubated with LCM or NCM for 16 hours. Live-cell images for GFP fluorescence were collected before and after incubation by confocal microscopy. 

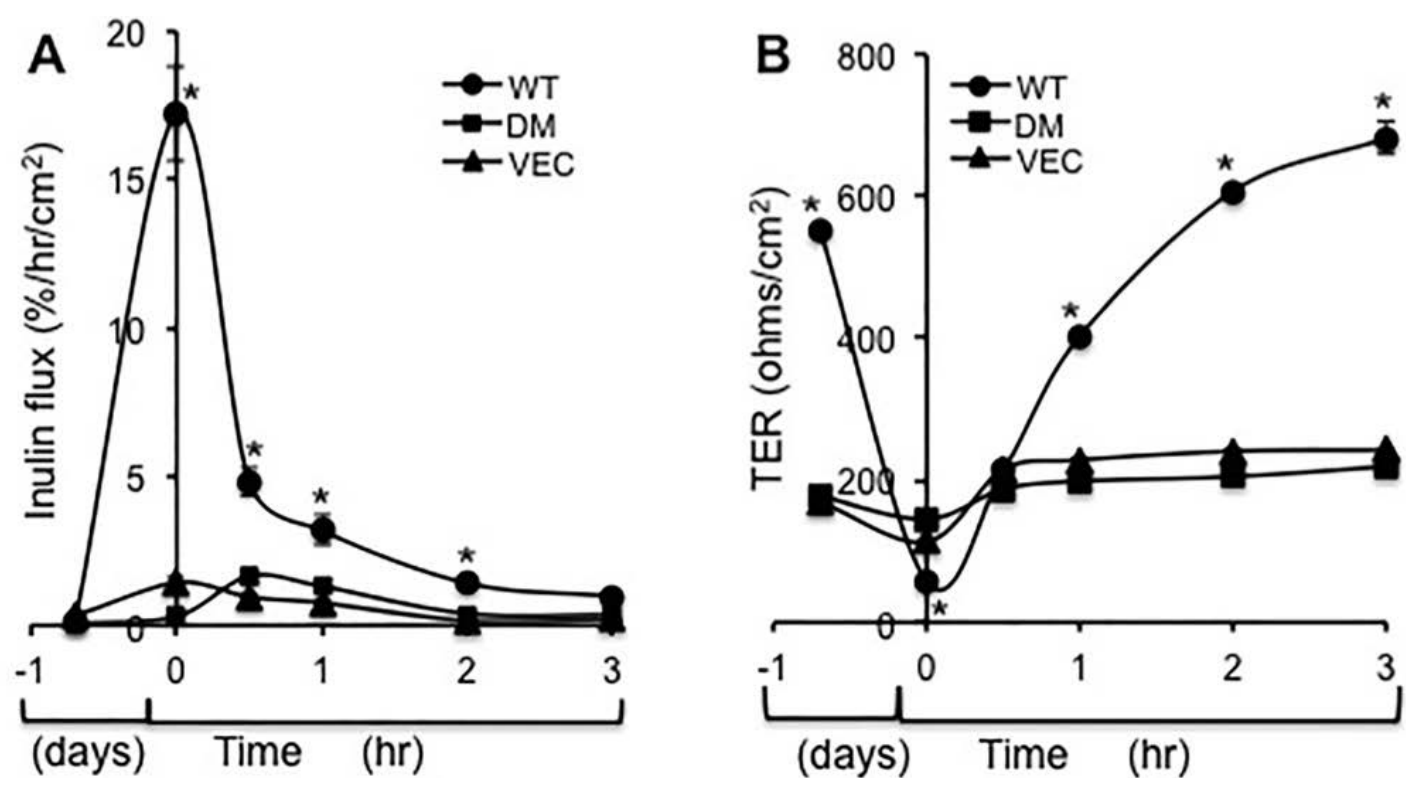

Figure 3-9. ORM Deletion Attenuates $\mathrm{Ca}^{2+}$ Depletion-induced Barrier Dysfunction.

A \& B: $\mathrm{Ocl}^{\mathrm{WT}}$ and $\mathrm{Ocl}^{\mathrm{DM}}$ cell monolayers on transwell inserts were incubated with LCM for 16 hours followed by incubation with NCM for up to $3 \mathrm{hr}$. TER (A) and FITC-inulin flux (B) were measured at varying times. Values are mean $\pm \operatorname{sem}(n=6)$. Asterisks indicate the values for $\mathrm{Ocl}^{\mathrm{WT}}$ cell monolayers that are significantly $(\mathrm{p}<0.05)$ different from corresponding values for $\mathrm{Ocl}^{\mathrm{DM}}$ and $\mathrm{Vec}$ cell monolayers. 


\section{Deletion of ORM Confers Resistance to Disruption of Apical Junctional Complex and Cytoskeleton}

Tight junctions and adherens junctions form key components of the apical junctional complex that are directly or indirectly connected to the actin cytoskeleton via scaffold proteins proteins and actin linkers. Here we examined the effect of ORM deletion on $\mathrm{Ca}^{2+}$ depletion-mediated changes in the apical junctional complex. Confluent cell monolayers were incubated in LCM and fixed in 3\% paraformaldehyde. The fixed monolayers were labeled for TJ components: GFP-occludin and ZO-1, AJ components: E-cadherin and b-catenin and cytoskeleton: actin and tubulin.

$\mathrm{Ca}^{2+}$ depletion in OD-Ocl ${ }^{\mathrm{WT}}$ cells induced redistribution of occludin and ZO-1 indicating TJ disruption (Figure 3-10A) as well as redistribution of E-Cadherin and bcatenin indicating the disruption of AJ (Figure 3-10B). However, OD-Ocl ${ }^{\mathrm{DM}}$ cells were resistant to both $\mathrm{TJ}$ and $\mathrm{AJ}$ disruption. $\mathrm{Ca}^{2+}$ depletion also induced disassociation of actin and tubulin in $\mathrm{Ocl}^{\mathrm{WT}}$ but not in $\mathrm{Ocl}^{\mathrm{DM}}$ monolayers (Figure 3-10C). These results together confirmed that ORM is required for mediating $\mathrm{Ca}^{2+}$ depletion-induced disruption of TJ, $\mathrm{AJ}$ and cytoskeleton.

\section{ORM Deletion Attenuates TJ and AJ Disruption Induced by EGTA-based $\mathrm{Ca}^{2+}$ Depletion}

EGTA is a $\mathrm{Ca}^{2+}$ chelating agent widely used to deplete extracellular $\mathrm{Ca}^{2+}$ in calcium switch assay. Because $\mathrm{Ocl}^{\mathrm{DM}}$ cells were resistant to low $\mathrm{Ca}^{2+}$-mediated TJ disruption, we used EGTA to induce TJ disassembly. Confluent cell monolayers were exposed to $4 \mathrm{mM}$ EGTA and TER and inulin-flux were measured at varying time points. Fixed monolayers were labeled for GFP, ZO-1, E-cadherin and $\beta$-catenin. The images were collected using a confocal microscope.

In Ocl ${ }^{\mathrm{WT}}$ cells, EGTA induced a decrease in TER by $5 \mathrm{~min}$, which was delayed in the case of $\mathrm{Ocl}^{\mathrm{DM}}$ cells (Figure 3-11A). EGTA treatment led to an increase in inulin-flux in cells Ocl ${ }^{\mathrm{WT}}$ cells, which was significantly delayed in $\mathrm{Ocl}^{\mathrm{DM}}$ cells (Figure 3-11B). GFP-occludin and ZO-1 started to show disassembly as early as 5 min, which was prominent by $30 \mathrm{~min}$. On the other hand, $\mathrm{Ocl}^{\mathrm{DM}}$ cells showed significant delay in occludin disassembly (Figure 3-11C). Similarly differences in AJ redistribution were observed (Figure 3-11D). These data confirmed that ORM deletion confers resistance to $\mathrm{Ca}^{2+}$ depletion-mediated occludin disassembly.

\section{Deletion of ORM Does Not Prevent CytochalasinD-mediated Disruption of TJ and Barrier Dysfunction}

Disruption of actin cytoskeleton also disrupts TJ. To examine whether the resistance to TJ disruption in the absence of ORM was specific to $\mathrm{Ca}^{2+}$ depletion, here we 


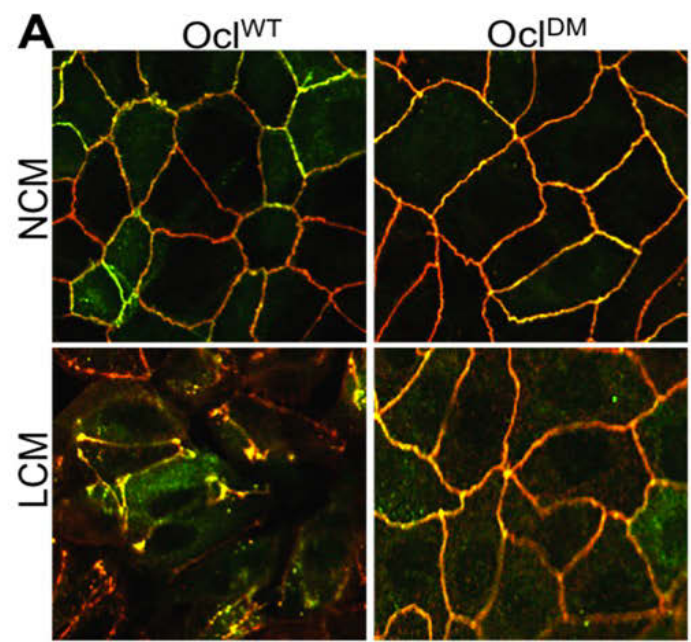

Green: GFP-occludin/Red: ZO-1

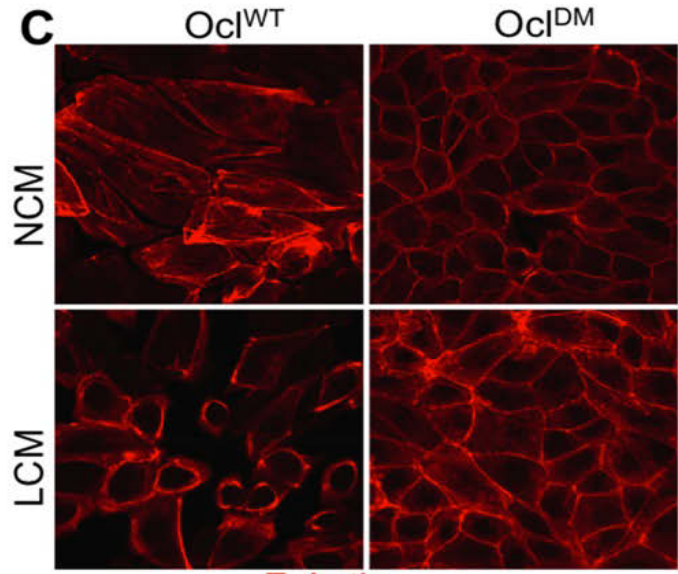

F-Actin

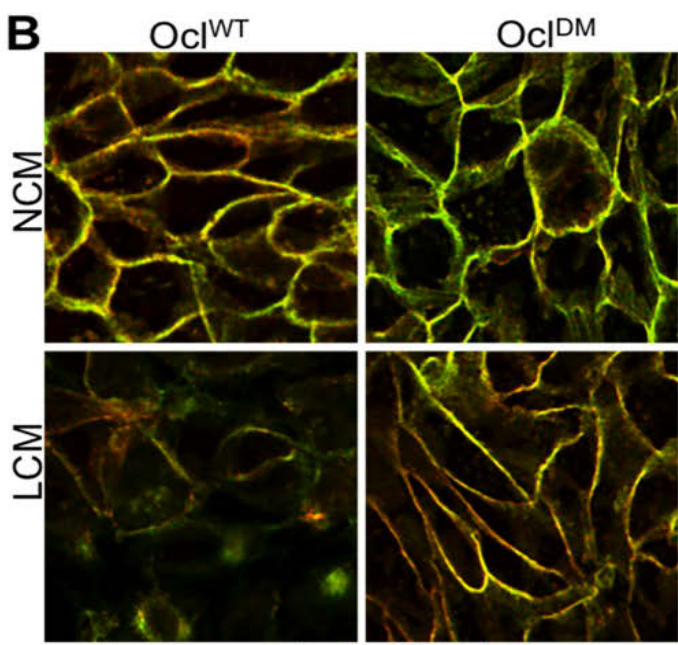

Green: E-Cad/Red: $\beta$-cat

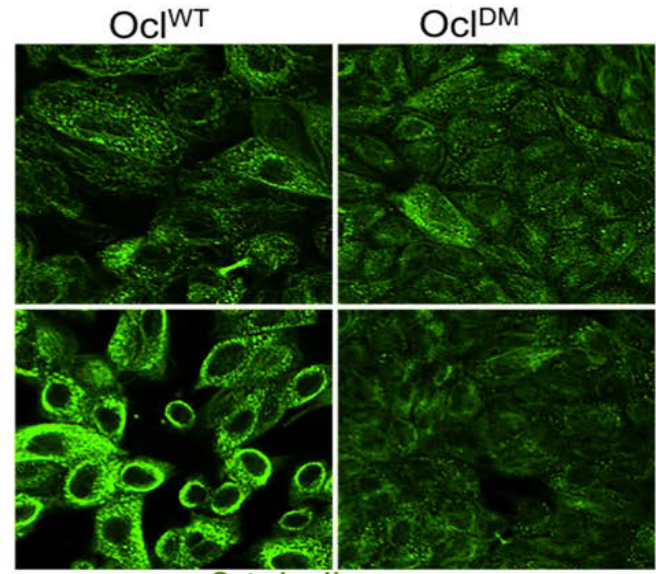

$\beta$-tubulin

Figure 3-10. ORM Deletion Attenuates $\mathrm{Ca}^{2+}$ Depletion-mediated Disruption of Apical Junctional Complex and Cytoskeleton.

A, B \& C: $\mathrm{Ocl}^{\mathrm{WT}}$ and $\mathrm{Ocl}^{\mathrm{DM}}$ cell monolayers were incubated with LCM or NCM for 16 hr. Fixed cell monolayers were stained for TJ proteins GFP-occludin (green) and ZO-1 (red) (A), AJ proteins E-cadherin (green) and $\beta$-Catenin (red) (B) and cytoskeletal proteins $\mathrm{F}$-actin (red) and $\beta$-tubulin (green) $(\mathbf{C})$. 

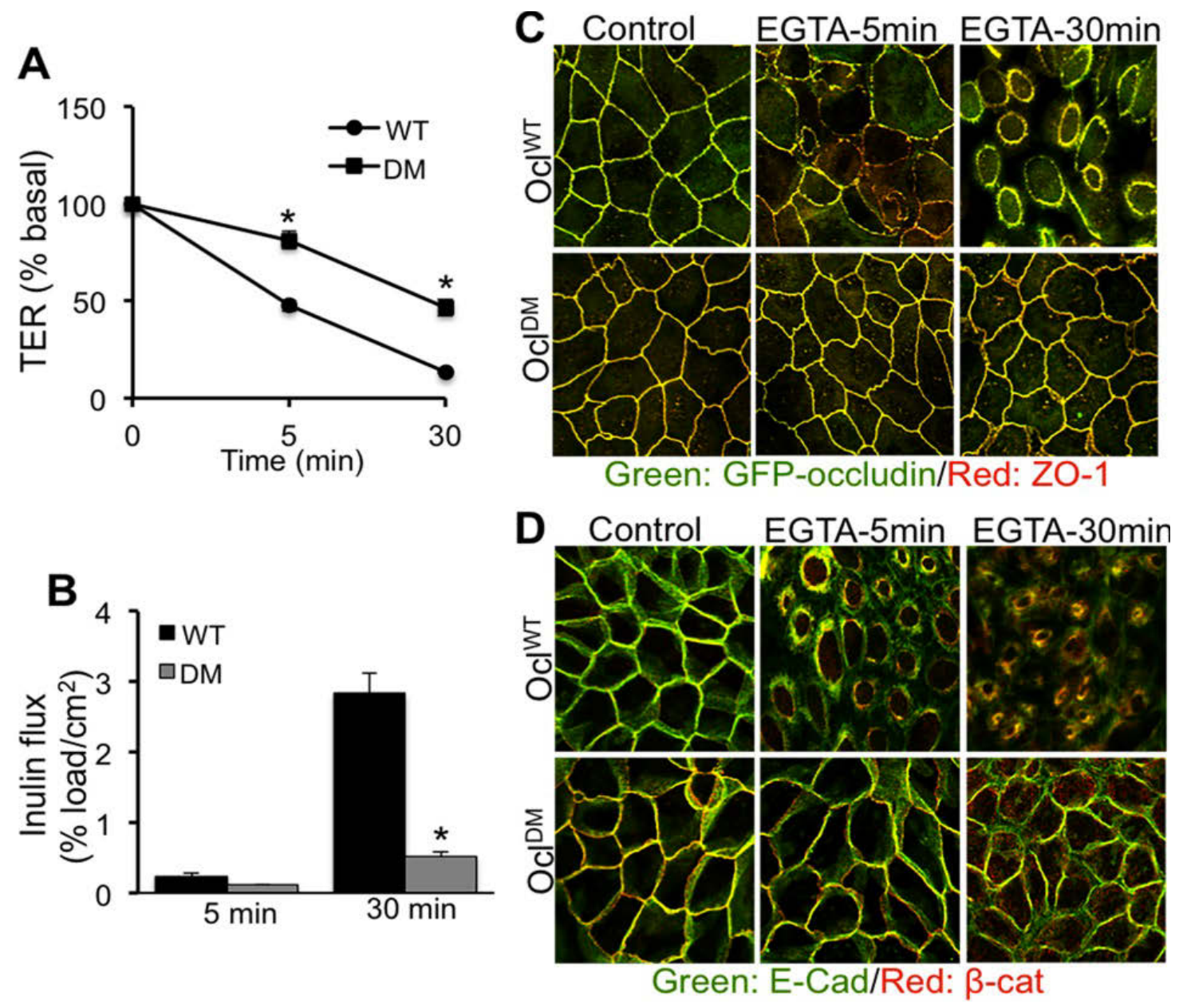

Figure 3-11. ORM Deletion Attenuates EGTA-mediated TJ and AJ Disruption.

A \& B: $\mathrm{Ocl}^{\mathrm{WT}}$ and $\mathrm{Ocl}^{\mathrm{DM}}$ cell monolayers on transwell inserts were incubated with $4 \mathrm{mM}$ EGTA. TER (A) and FITC-inulin flux (B) were measured at varying times. Values are mean $\pm \operatorname{sem}(n=6)$. Asterisks indicate the values for $\mathrm{Ocl}^{\mathrm{WT}}$ cell monolayers that are significantly $(\mathrm{p}<0.05)$ different from corresponding values for $\mathrm{Ocl}^{\mathrm{DM}}$ and Vec cell monolayers. C \& D: Cell monolayers fixed at varying times after EGTA administration were co-stained for GFP (Green) \& ZO-1 (Red) (C) or E-cadherin (Green) \& $\beta$-catenin (Red) (D). 
employed another method to disrupt TJ. Confluent cell monolayers in transwells were treated with cytochalasin D $(2 \mu \mathrm{g} / \mathrm{ml})$. Barrier function was assessed by measuring TER and inulin flux at various time points. Live imaging was also performed by exposing cells to cytochalasin D.

Incubation of $\mathrm{Ocl}^{\mathrm{WT}}, \mathrm{Ocl}^{\mathrm{DM}}$ and Vec cell monolayers with cytochalasin-D resulted in a drop in TER (Figure 3-12A) and increase in inulin-flux (Figure 3-12B). Live cell imaging of GFP fluorescence showed that cytochalasin-D induced disruption of occludin from the junctions in both $\mathrm{Ocl}^{\mathrm{WT}}$ and $\mathrm{Ocl}^{\mathrm{DM}}$ cell monolayers. All cells displayed characteristic appearance of discontinuous junctional occludin, typical of cytochalasin-D induced TJ disruption. These data together suggested that ORM is not required for mediating cytochalasin-D mediated barrier dysfunction.

\section{Deletion of ORM Does Not Prevent Osmotic Stress-mediated Disruption of TJ and Barrier Dysfunction}

Another method to disrupt TJ was employed to investigate the specificity of ORM deletion-induced resistance to $\mathrm{Ca}^{2+}$ depletion-mediated TJ disruption. Confluent cell monolayers in $60 \mathrm{~mm}$ dishes and $12 \mathrm{~mm}$ transwells were exposed to 600mOsM DMEM to induce osmotic stress. Barrier dysfunction was monitored by measuring TER and inulin-flux and live-cell imaging for GFP at varying time points. Fixed monolayers were stained for GFP and ZO-1 by immunofluorescence method.

Exposure to osmotic stress induced increase in inulin permeability in $\mathrm{Ocl}^{\mathrm{WT}}$, $\mathrm{Ocl}^{\mathrm{DM}}$ and Vec cell monolayers (Figure 3-13A) Live cell imaging for GFP showed osmotic stress-mediated junctional redistribution of GFP-occludin in both $\mathrm{Ocl}^{\mathrm{WT}}$ and $\mathrm{Ocl}^{\mathrm{DM}}$ cell monolayers (Figure 3-13B). Stain from fixed cell monolayers for GFP and ZO-1 indicated that osmotic stress induced redistribution of GFP-occludin and ZO-1 from the intercellular junctions in both $\mathrm{Ocl}^{\mathrm{WT}}$ and $\mathrm{Ocl}^{\mathrm{DM}}$ cell monolayers (Figure 313C).

\section{Phoshorylation of ORM on T403/403 and Y398/402 Determines Dynamic Properties of Occludin}

Previous studies showed that the phosphorylation of threonine residues (T403/404) enhances TJ assembly while phosphorylation of tyrosine residues (Y398/402) negatively regulates TJ assembly. To investigate the role of ORM phosphorylation in occludin dynamics we generated OD-mutant clones expressing GFP-Ocl ${ }^{\mathrm{T} 403 / 404 \mathrm{~A}}$, GFP$\mathrm{Ocl}^{\mathrm{T} 403 / 404 \mathrm{D}}$, GFP-Ocl ${ }^{\mathrm{Y} 398 / 402 \mathrm{~A}}$ and GFP-Ocl ${ }^{\mathrm{Y} 398 / 402 \mathrm{D}}$. Confluent cell monolayers were incubated in LCM and live images were captured at varying time points. FRAP analyses was performed in these cells to assess the role of ORM phosphorylation on occludin mobility. 

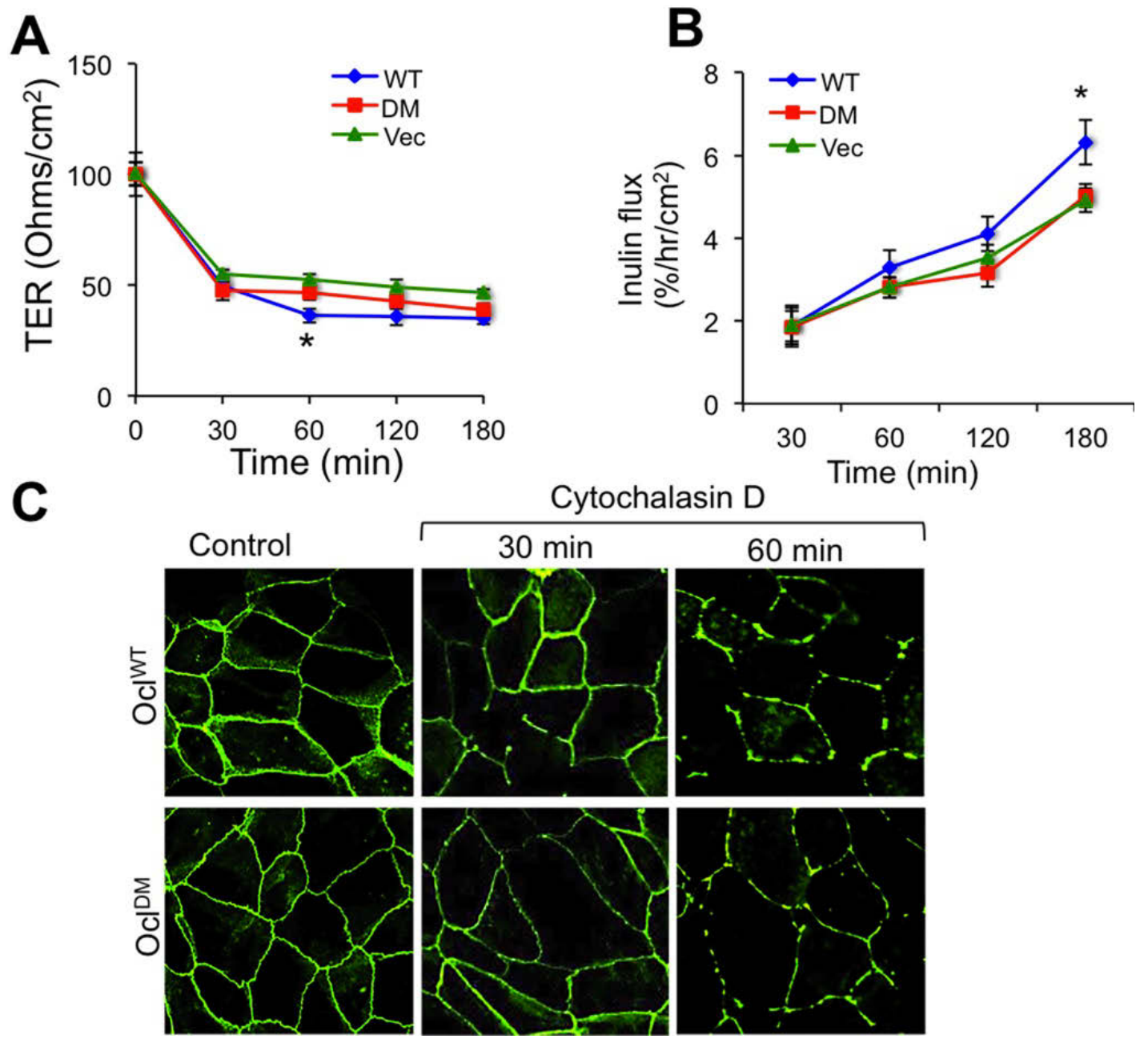

Figure 3-12. ORM Deletion Does Not Prevent Cytochalasin-mediated TJ Disruption and Barrier Dysfunction.

A \& B: $\mathrm{Ocl}^{\mathrm{WT}}, \mathrm{Ocl}^{\mathrm{DM}}$ and Vec cell monolayers on transwell inserts were incubated with Cytochalasin-D. TER (A) and FITC-inulin flux (B) were measured at varying times. Values are mean $\pm \operatorname{sem}(n=6)$. C: Live-cell images for GFP fluorescence were captured at varying times. 

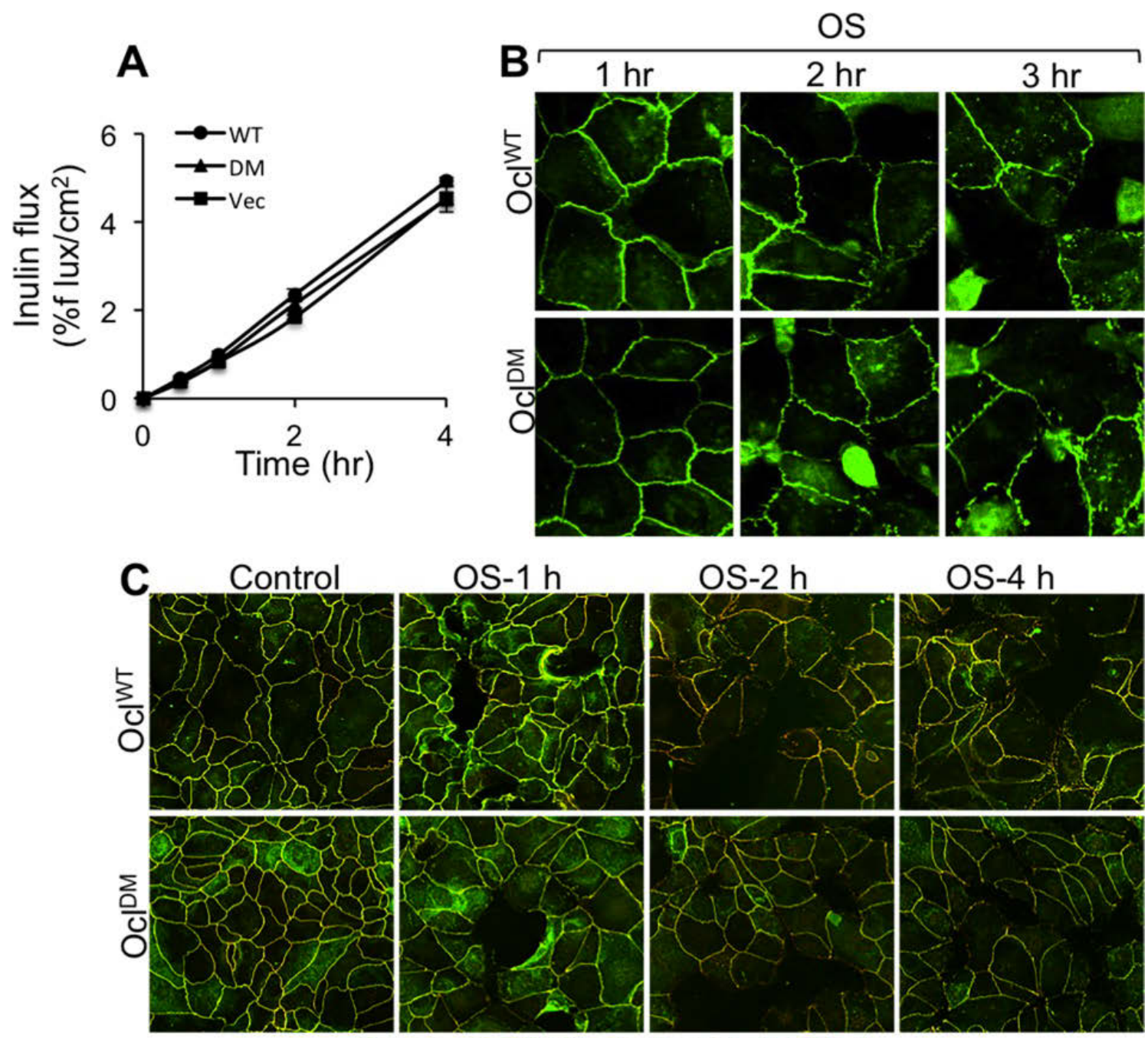

Green: GFP-Occludin/ Red: ZO-1

Figure 3-13. ORM Deletion Does Not Prevent Osmotic Stress-mediated TJ Disruption and Barrier Dysfunction.

A: $\mathrm{Ocl}^{\mathrm{WT}}, \mathrm{Ocl}^{\mathrm{DM}}$ and Vec cell monolayers were incubated with hyperosmotic medium and FITC-inulin flux was measured at varying times. Values are mean $\pm \operatorname{sem}(n=6)$. B: $\mathrm{Ocl}^{\mathrm{WT}}$ and $\mathrm{Ocl}^{\mathrm{DM}}$ cell monolayers were incubated with hyperosmotic medium $(0.35 \mathrm{M}$ mannitol) and live images for GFP fluorescence were captured at varying times. C: Fixed cell monolayers were co-stained for GFP (Green) and ZO-1 (Red). 
Live cell imaging of GFP fluorescence showed that incubation with LCM for 24 $\mathrm{hr}$ resulted in redistribution of GFP-Ocl ${ }^{\mathrm{T} 403 / 404 \mathrm{D}}$ from the intercellular junctions similar to GFP-Ocl $^{\text {WT }}$. However, redistribution of GFP-Ocl ${ }^{\text {T403/404A }}$ was not altered with $\mathrm{Ca}^{2+}$ depletion similar to that of GFP-Ocl ${ }^{\mathrm{DM}}$. On the other hand, incubation with LCM induced partial redistribution of both GFP-Ocl ${ }^{\mathrm{Y} 398 / 402 \mathrm{D}}$ and GFP-Ocl $^{\mathrm{Y} 398 / 402 \mathrm{~A}}$ (Figure 3-14). These observations indicated that ORM contributes to occludin dynamics in a phosphorylation dependent mechanism. FRAP analysis demonstrated that the percent mobile fraction of GFP fluorescence $\mathrm{Ocl}^{\mathrm{T}}{ }^{\mathrm{H} 3 / 404 \mathrm{D}}$ cell monolayers was similar to that of Ocl ${ }^{\mathrm{WT}}$, whereas mobile fractions of $\mathrm{Ocl}^{\mathrm{DM}}, \mathrm{Ocl}^{\mathrm{T} 403 / 404 \mathrm{~A}}, \mathrm{Ocl}^{\mathrm{Y398/402 \textrm {D }}}$ and $\mathrm{Ocl}^{\mathrm{Y398/402A}}$ were significantly low compared to that of $\mathrm{Ocl}^{\mathrm{WT}}$ cell monolayers (Figure 3-15).

\section{Phosphorylation of ORM on T403/403 and Y398/402 Determines Dynamic Properties of TJ}

To investigate the role of ORM phosphorylation in TJ dynamics, mutant clones expressing GFP-Ocl ${ }^{\mathrm{DM}}$, GFP-Ocl ${ }^{\mathrm{T} 403 / 404 \mathrm{~A}}$, GFP-Ocl $^{\mathrm{T} 403 / 404 \mathrm{D}}$, GFP-Ocl $^{\mathrm{Y398} / 402 \mathrm{~A}}$ and GFP$\mathrm{Ocl}^{\mathrm{Y} 398 / 402 \mathrm{D}}$ and wild type cells expressing GFP-Ocl ${ }^{\mathrm{WT}}$ were grown on transwells. Confluent cell monolayers were incubated in LCM and barrier function was assessed. Fixed monolayers at varying time points were labeled for GFP and ZO-1.

Measurement of TER in these cell lines showed that, incubation with LCM for 16 hr results in a decrease in TER in $\mathrm{Ocl}^{\mathrm{WT}}$ and $\mathrm{Ocl}^{\mathrm{T} 403 / 404 \mathrm{D}}$ cell monolayers; these effects are significantly greater compared to those in $\mathrm{Ocl}^{\mathrm{DM}}, \mathrm{Ocl}^{\mathrm{T} 403 / 404 \mathrm{~A}}, \mathrm{Ocl}^{\mathrm{Y} 398 / 402 \mathrm{D}}$ and $\mathrm{Ocl}^{\mathrm{Y} 398 / 402 \mathrm{~A}}$ cell monolayers (Figure 3-16A). Inulin flux measurement, after $16 \mathrm{hr}$ incubation with LCM showed increased inulin permeability in both $\mathrm{Ocl}^{\mathrm{WT}}$ and $\mathrm{Ocl}^{\mathrm{T} 403 / 404 \mathrm{D}}$ cell monolayers. LCM-mediated inulin permeability was low in $\mathrm{Ocl}^{\mathrm{DM}}$ and $\mathrm{Ocl}^{\mathrm{T} 403 / 404 \mathrm{~A}}$ cell monolayers and moderate in $\mathrm{Ocl}^{\mathrm{Y398/402D}}$ and $\mathrm{Ocl}^{\mathrm{Y} 398 / 402 \mathrm{~A}}$ cell monolayers (Figure 3-16B). Immunofluorescent staining of fixed monolayers for GFP and ZO-1 showed that LCM induced redistribution of both occludin and ZO-1 from intercellular junctions in $\mathrm{Ocl}^{\text {WT }}$ and OclT403/404D cell monolayers but not in $\mathrm{Ocl}^{\mathrm{DM}}$ and $\mathrm{Ocl}^{\mathrm{T} 403 / 404 \mathrm{~A}}$ cell monolayers. $\mathrm{Ca}^{2+}$ depletion had a significantly low effect on redistribution of occludin and ZO-1 in both $\mathrm{Ocl}^{\mathrm{Y} 398 / 402 \mathrm{D}}$ and $\mathrm{Ocl}^{\mathrm{Y} 398 / 402 \mathrm{~A}}$ cell monolayers compared to that in $\mathrm{Ocl}^{\mathrm{WT}}$ cell monolayers (Figure 3-17). These data together suggested that ORM confers dynamic properties to $\mathrm{TJ}$ in a phosphorylation dependent mechanism.

\section{Tyrosine Phosphorylation Plays a Role in $\mathrm{Ca}^{2+}$ Depletion-mediated TJ Disruption}

Oxidative stress and osmotic stress mediated TJ disruption involves tyrosine phosphorylation of occludin on residues Y398 and Y402. However, very little is known about the role of tyrosine phosphorylation in $\mathrm{Ca}^{2+}$ switch assay. From the studies using mutation of tyrosine residues in ORM, it was clear that tyrosine phosphorylation is also involved in LCM-mediated TJ disruption. In order to further validate the role of tyrosine phosphorylation in $\mathrm{Ca}^{2+}$ depletion-mediated TJ disruption, MDCK cells grown on transwells were pretreated with either genistein or DMSO for $1 \mathrm{hr}$. Cells were incubated 


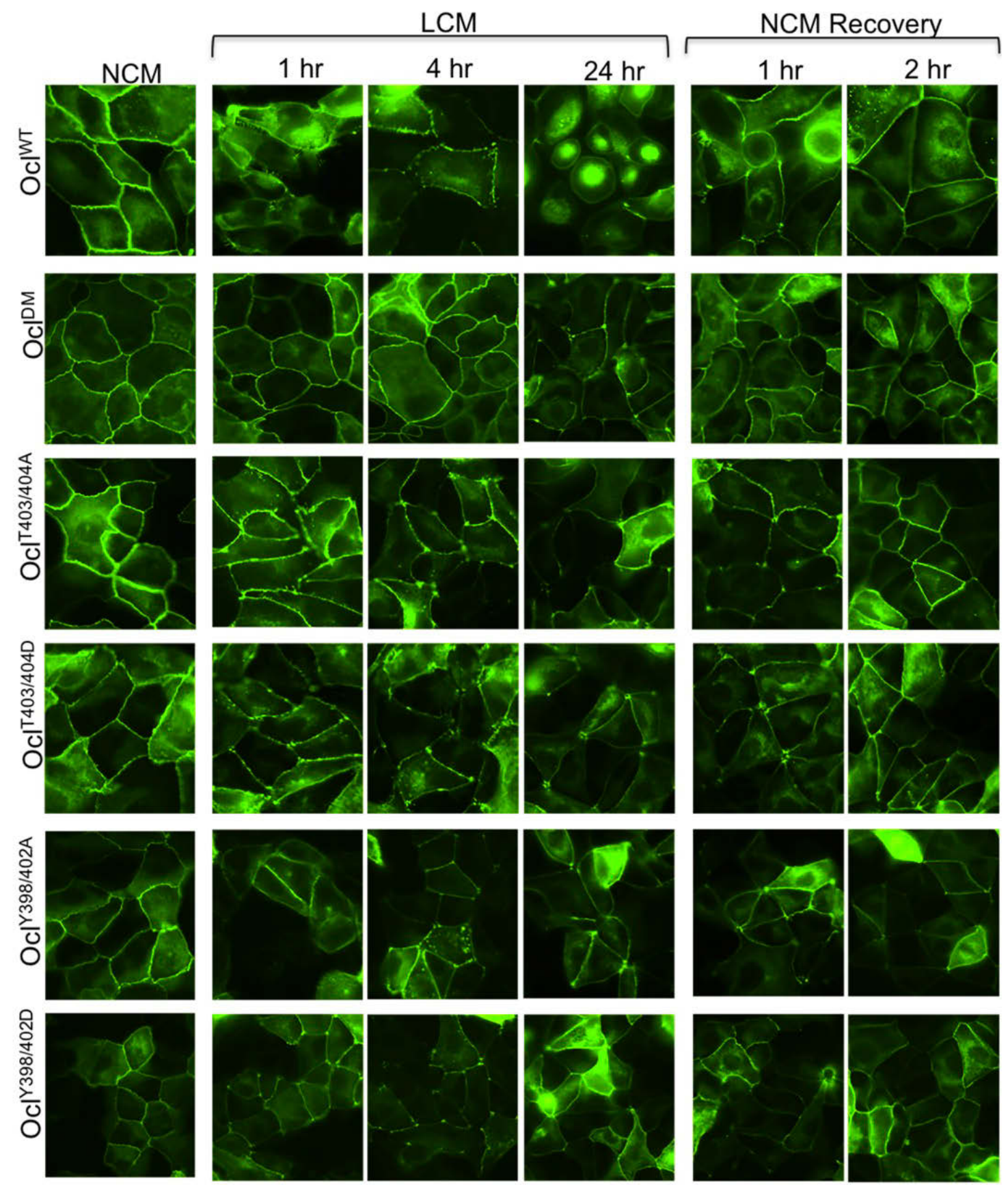

Figure 3-14. ORM Confers Dynamic Properties to Occludin in a Phosphorylationdependent Manner.

$\mathrm{Ocl}^{\mathrm{WT}}, \mathrm{Ocl}^{\mathrm{DM}}, \mathrm{Ocl}^{\mathrm{T} 403 / 404 \mathrm{~A}}, \mathrm{Ocl}^{\mathrm{T} 403 / 404 \mathrm{D}}, \mathrm{Ocl}^{\mathrm{Y} 398 / 402 \mathrm{~A}}$ and $\mathrm{Ocl}^{\mathrm{Y} 398 / 402 \mathrm{D}}$ cell monolayers were incubated with LCM for $24 \mathrm{hr}$ and in NCM for $2 \mathrm{hr}$. Live-cell images for GFP fluorescence were collected before and after incubation. 


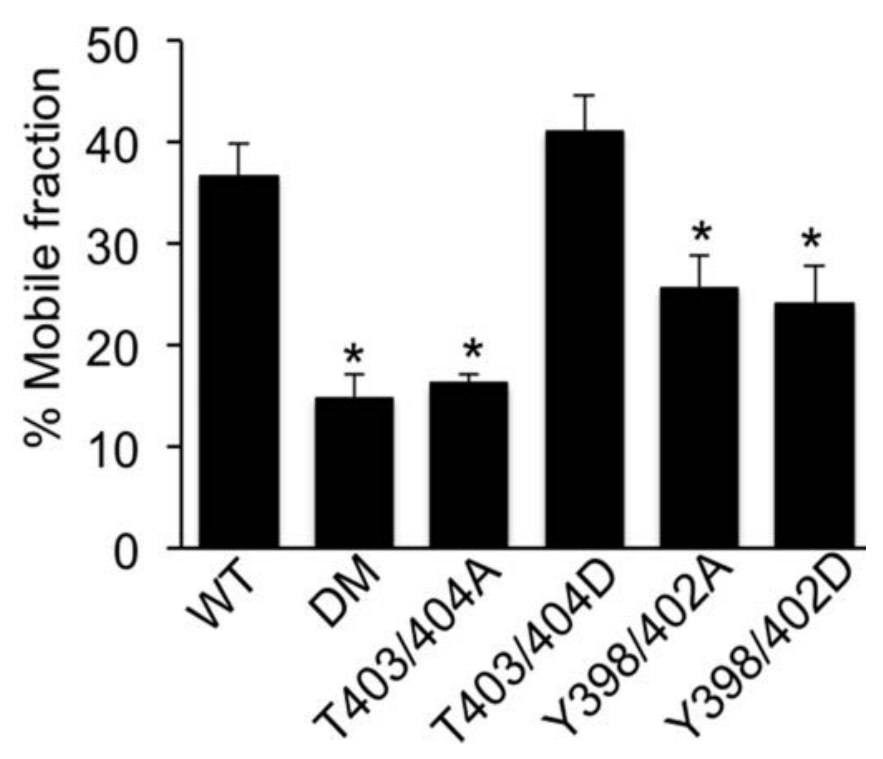

Figure 3-15. ORM Phosphorylation Determines Mobility of Occludin at TJ.

FRAP analysis of GFP in $\mathrm{Ocl}^{\mathrm{WT}}, \mathrm{Ocl}^{\mathrm{DM}}, \mathrm{Ocl}^{\mathrm{T} 403 / 404 \mathrm{~A}}, \mathrm{Ocl}^{\mathrm{T} 403 / 404 \mathrm{D}}, \mathrm{Ocl}^{\mathrm{Y} 398 / 402 \mathrm{~A}}$ and $\mathrm{Ocl}^{\mathrm{Y} 398 / 402 \mathrm{D}}$ cell monolayers. Time-lapse images were collected before and after photo bleaching of ROI at intercellular junctions. Fluorescence intensity was measured and \% mobile fraction was calculated. 

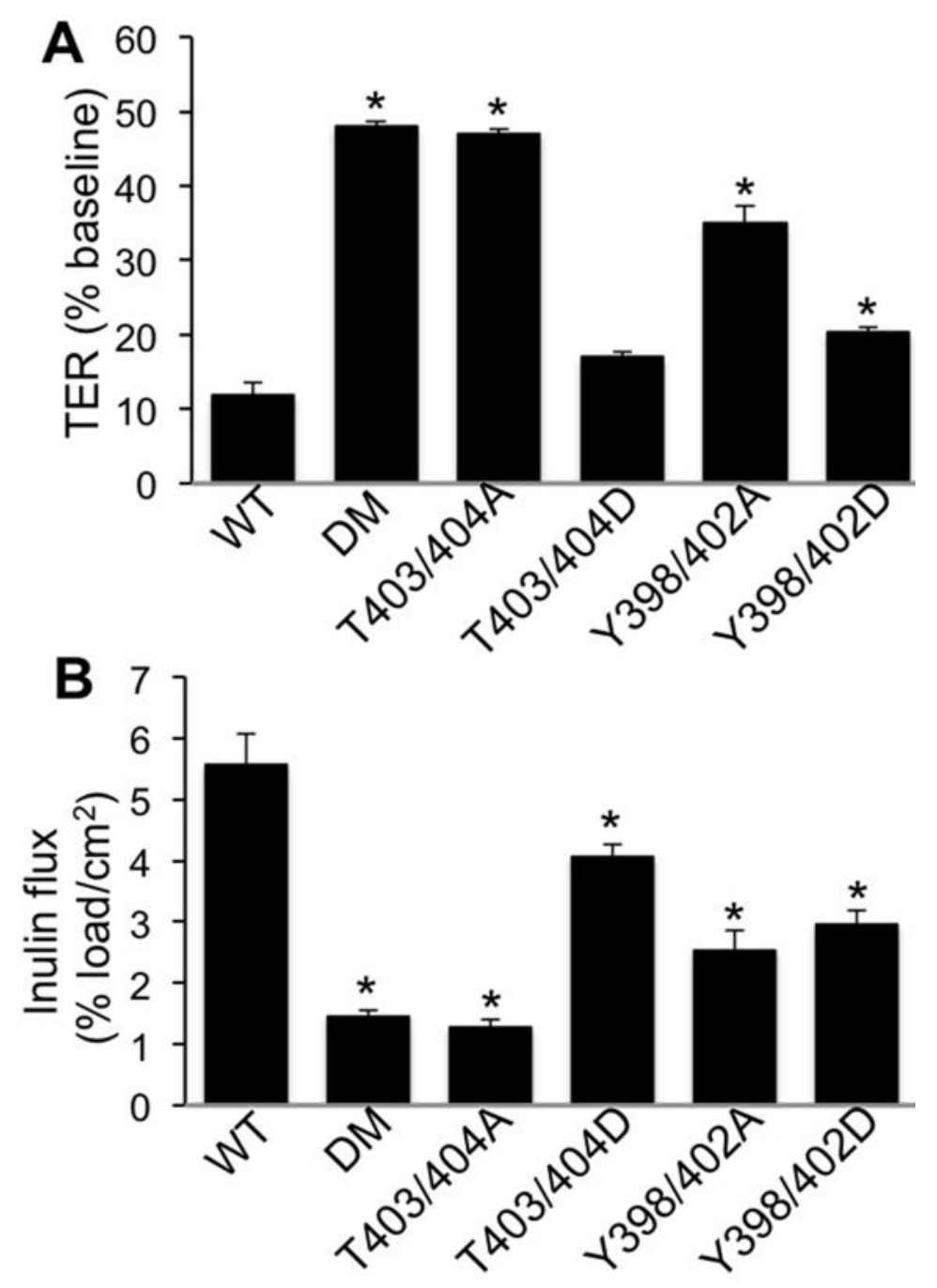

Figure 3-16. ORM Determines Barrier Function in a Phosphorylation-dependent Mechanism.

A \& B: $\mathrm{Ocl}^{\mathrm{WT}}, \mathrm{Ocl}^{\mathrm{DM}}, \mathrm{Ocl}^{\mathrm{T} 403 / 404 \mathrm{~A}}, \mathrm{Ocl}^{\mathrm{T} 403 / 404 \mathrm{D}}, \mathrm{Ocl}^{\mathrm{Y398/402A}}$ and $\mathrm{Ocl}^{\mathrm{Y398/402 \textrm {D }}}$ cell monolayers on transwell inserts were incubated with LCM for 16 hours. TER (A) and FITC-inulin flux $(\mathbf{B})$ were measured at varying times. Values are mean $\pm \operatorname{sem}(n=6)$. Asterisks indicate the values for $\mathrm{Ocl}^{\mathrm{WT}}$ cell monolayers that are significantly $(\mathrm{p}<0.05)$ different from corresponding values for $\mathrm{Ocl}^{\mathrm{DM}}$ and Vec cell monolayers. 


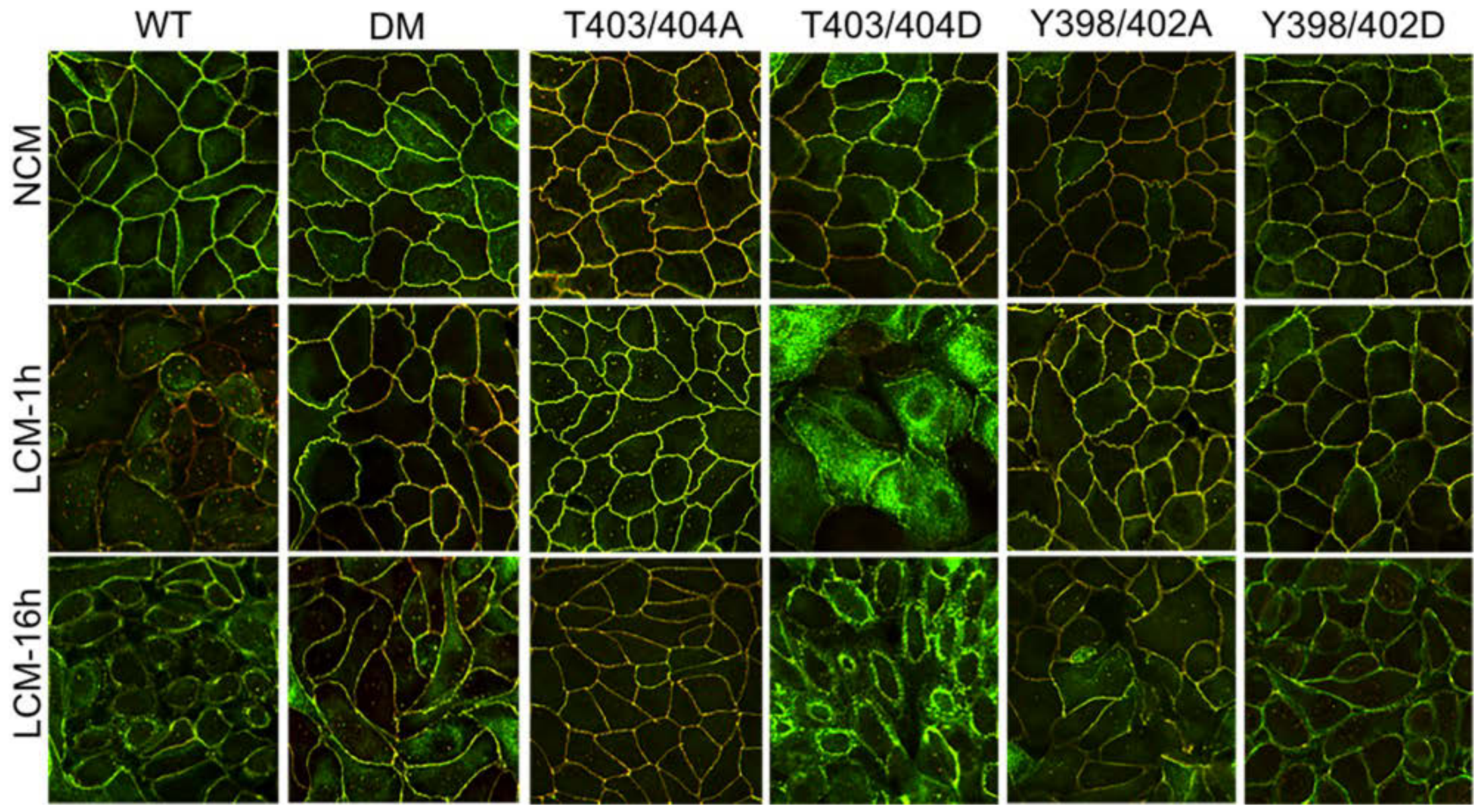

Figure 3-17. ORM Confers Dynamic Properties to TJ in a Phosphorylation-dependent Mechanism.

$\mathrm{Ocl}^{\mathrm{WT}}, \mathrm{Ocl}^{\mathrm{DM}}, \mathrm{Ocl}^{\mathrm{T} 403 / 404 \mathrm{~A}}, \mathrm{Ocl}^{\mathrm{T} 403 / 404 \mathrm{D}}, \mathrm{Ocl}^{\mathrm{Y} 398 / 402 \mathrm{~A}}$ and $\mathrm{Ocl}^{\mathrm{Y} 398 / 402 \mathrm{D}}$ cell monolayers were incubated with LCM for 16 hr. Cell monolayers fixed at $1 \mathrm{hr}$ and $16 \mathrm{hr}$ were stained for GFP (Green) and ZO-1 (Red). 
in LCM and barrier function was monitored by measuring TER and inulin-flux. Cell monolayers fixed at varying time points were labeled for occludin and ZO-1. Incubation with LCM induced a decrease in TER (Figure 3-18A) and an increase in inulin-flux within $1 \mathrm{hr}$ (Figure 3-18B), whereas treatment with genistein blocked the effect of LCM. Confocal images for occludin and ZO-1 showed that LCM induced redistribution of both occludin and ZO-1 from intercellular junctions into the intracellular compartment whereas cell monolayers treated with genistein were resistant to LCM-mediated redistribution of occludin and ZO-1 (Figure 3-18C). These data confirm that tyrosine phosphorylation plays a significant role in $\mathrm{Ca}^{2+}$ depletion-mediated TJ disruption.

\section{Specific Aim 2: To Determine That ORM-mediated Protein Interactions Are Involved in TJ Modulation}

\section{ORP Permeability}

In order to study the role of ORM in TJ modulation, we designed a synthetic peptide based on the occludin regulatory region. It has a biotin tag at its $\mathrm{N}$-terminus and a TAT domain at its $\mathrm{C}$-terminus. The biotin tag was intended to aid in the pull down assays, while the TAT sequence was added to aid in the permeation of ORP into cells. The peptide design is as shown in (Figure 3-19A). A scrambled peptide was used as a control.

First we investigated the permeation of ORP in Caco-2 cells. Confluent cell monolayers were treated with ORP. Fixed cell monolayers were immunostained for biotin and occludin. We then tested ORP permeability in mouse intestine. Isolated intestinal loops were injected with either saline or ORP and incubated for $1 \mathrm{hr}$. Cryosections were labeled for ZO-1, biotin and nucleus.

The confocal images of Caco-2 cell monolayers showed that ORP does permeate into the cells in a dose dependent manner, as seen by an increase in the intracellular Cy3streptavidin (Red), stained for biotin (Figure 3-19B). The images of intestinal loop injected with ORP showed a similar permeation of ORP into intestinal mucosa as seen by the stain for biotin (Red) (Figure 3-19C). These data together showed that ORP has the ability to permeate into cells.

\section{ORP Enhances TJ Assembly During $\mathrm{Ca}^{2+}$ Switch Assay in Caco-2 Cells}

The biological effect of ORP was examined in Caco-2 cells. $\mathrm{Ca}^{2+}$ switch assay was performed in confluent cell monolayers in the presence of the peptide. EGTA was used to deplete extracellular $\mathrm{Ca}^{2+}$ from the media and induce TJ disassembly. During recovery, ORP was added to normal $\mathrm{Ca}^{2+}$ containing media. Inulin flux was measured at varying time points. Cell monolayers fixed $1 \mathrm{hr}$ after calcium switch were double labeled for occludin and ZO-1. 



Green: Occludin/Red: ZO-1

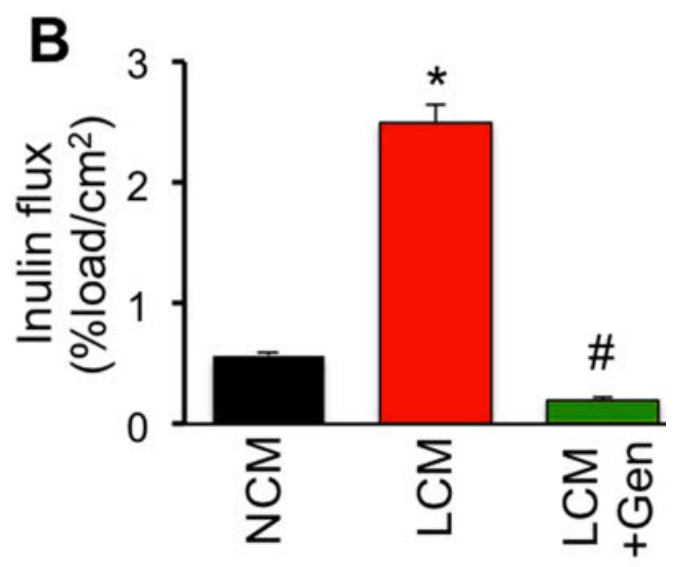

LCM



Figure 3-18. $\quad \mathrm{Ca}^{2+}$ Depletion-induced TJ Disruption Involves Tyrosine Phosphorylation.

A \& B: MDCK cell monolayers on transwells were treated with $100 \mu \mathrm{M}$ genistein. TER (A) and FITC-inulin flux (B) were measured after $1 \mathrm{hr}$. C: Cell monolayers treated with genistein were fixed at $1 \mathrm{hr}$ after incubation in LCM and co-stained for GFP (Green) and ZO-1 (Red). 




Figure 3-19. ORP Sequence and Its Permeability.

A: ORP is comprised has an N-terminal biotin tag and a C-terminal TAT domain.

ScrORP has a scrambled sequence. B: Confluent cell monolayers were treated with $3 \mu \mathrm{M}$ and $10 \mu \mathrm{M}$ ORP. Fixed cell monolayers were immunostained with anti-occludin antibody and Cy3-conjugated streptavidin. C: Isolated intestinal loops from C57BL/6 mice were injected with either saline or $10 \mu \mathrm{M}$ ORP and incubated in DMEM at $37^{\circ} \mathrm{C}$ for $1 \mathrm{hr}$. Cryosections of the loops were fixed and immunostained for ZO-1 (green), biotin (red) and nucleus (blue). 
$\mathrm{Ca}^{2+}$ depletion-induced inulin permeability was seen to decline during $\mathrm{Ca}^{2+}$ switch-mediated recovery, indicating TJ assembly. This decrease in inulin-flux was enhanced by the presence of ORP in a dose-dependent manner (Figure 3-20A). Confocal images showed that ORP enhanced junctional assembly of occludin and ZO-1 in ORP treated monolayers compared to that of the control (Figure 3-20B). These observations indicated that ORP has the ability to mimic ORM based interactions required for TJ assembly.

\section{ORP Attenuates Radiation-induced Barrier Dysfunction and TJ Disruption}

In order to study the effect of ORP on barrier function, a radiation model was used. Confluent monolayers of Caco- 2 cells in transwells were exposed to radiation (4 Gy). The barrier function post-radiation was monitored by measuring flux of FITC-inulin and TER.

To examine the effect of ORP on radiation induced barrier dysfunction, cells were pre-treated with ORP for $30 \mathrm{~min}$ and then exposed to radiation. TER and inulin-flux were measured. Cells were fixed at varying time points and immunostained for occludin, ZO-1 and nucleus.

Radiation induced a significant decrease in TER compared to the sham control (Figure 3-21A). There was a significant increase in inulin permeability in radiation treated cells compared to sham control (Figure 3-21B) indicating barrier dysfunction by radiation. However, when the cells were pre-incubated with ORP, 30 min prior to irradiation, barrier dysfunction was significantly attenuated compared to the scrambled control treatment as seen by relatively less drop in TER (Figure 3-21C) and decreased inulin flux (Figure 3-21D). The confocal images showed that ORP treatment attenuated radiation-induced disassembly of occludin and ZO-1 from TJ (Figure 3-22). These data together suggested that ORP attenuates radiation-induced barrier disruption.

\section{ORP as a Bait}

In order to identify the proteins that interact with ORM, ORP that has a biotin tag at its N-terminus was used as bait in pull down assays. Incubation of recombinant GST tagged occludin C-terminus (GST-Ocl-C) with tyrosine kinase led to phosphorylation of tyrosine residues, as seen by an immunoblot for phospho tyrosine $(33,34)$. Incubation of GST-Ocl-C with a serine/threonine kinase in vitro led to phosphorylation of threonine and serine residues and was evident from an immunoblot for phospho threonine and phospho serine (57).

Similarly, ORP was pre-phosphorylated in vitro by incubation with either active c-Src or CK2 in presence of ATP to yield tyrosine phosphorylated ORP (ORP-pY) or threonine-phosphorylated ORP (ORP-pS/T) respectively. These phosphopeptides were incubated with native protein extracts from Caco-2 cells and biotin was pulled down 



Green - Occludin; Red - ZO-1

Figure 3-20. ORP Enhances TJ Assembly.

Caco-2 cells seeded on transwells were treated with $4 \mathrm{mM}$ EGTA. Following this, the media was switched with regular media containing either $3 \& 10 \mu \mathrm{M}$ ORP or scrambled control along with FITC-inulin $(0.5 \mathrm{mg} / \mathrm{ml})$. A. Inulin flux was measured for $6 \mathrm{hr}$ during recovery. B. The monolayers were fixed $1 \mathrm{hr}$ after $\mathrm{Ca}^{2+}$ switch and immunostained for occludin (Green) and ZO-1 (Red). 




B


Figure 3-21. ORP Attenuates Radiation-induced Barrier Dysfunction.

A \& B: Confluent cell monolayers of Caco-2 cells in transwells were exposed to radiation (4 Gy). TER (A) and inulin-flux (B) were measured at varying time points. $\mathbf{C} \boldsymbol{\&}$ D: Cell monolayers were pre-treated with ORP for $30 \mathrm{~min}$ and then exposed to radiation (4 Gy). TER (C) and inulin-flux (D) were measured post-radiation. 

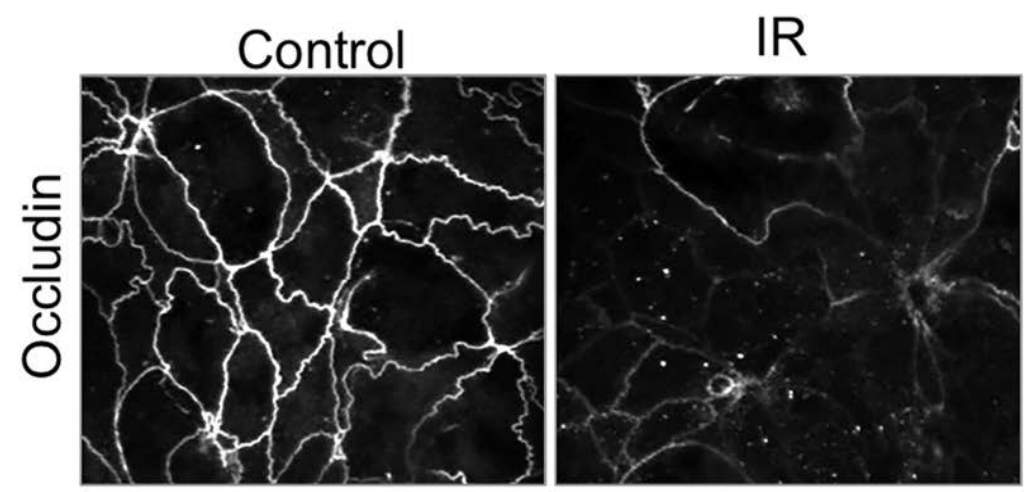

IR-ORP $(10 \mu \mathrm{M})$
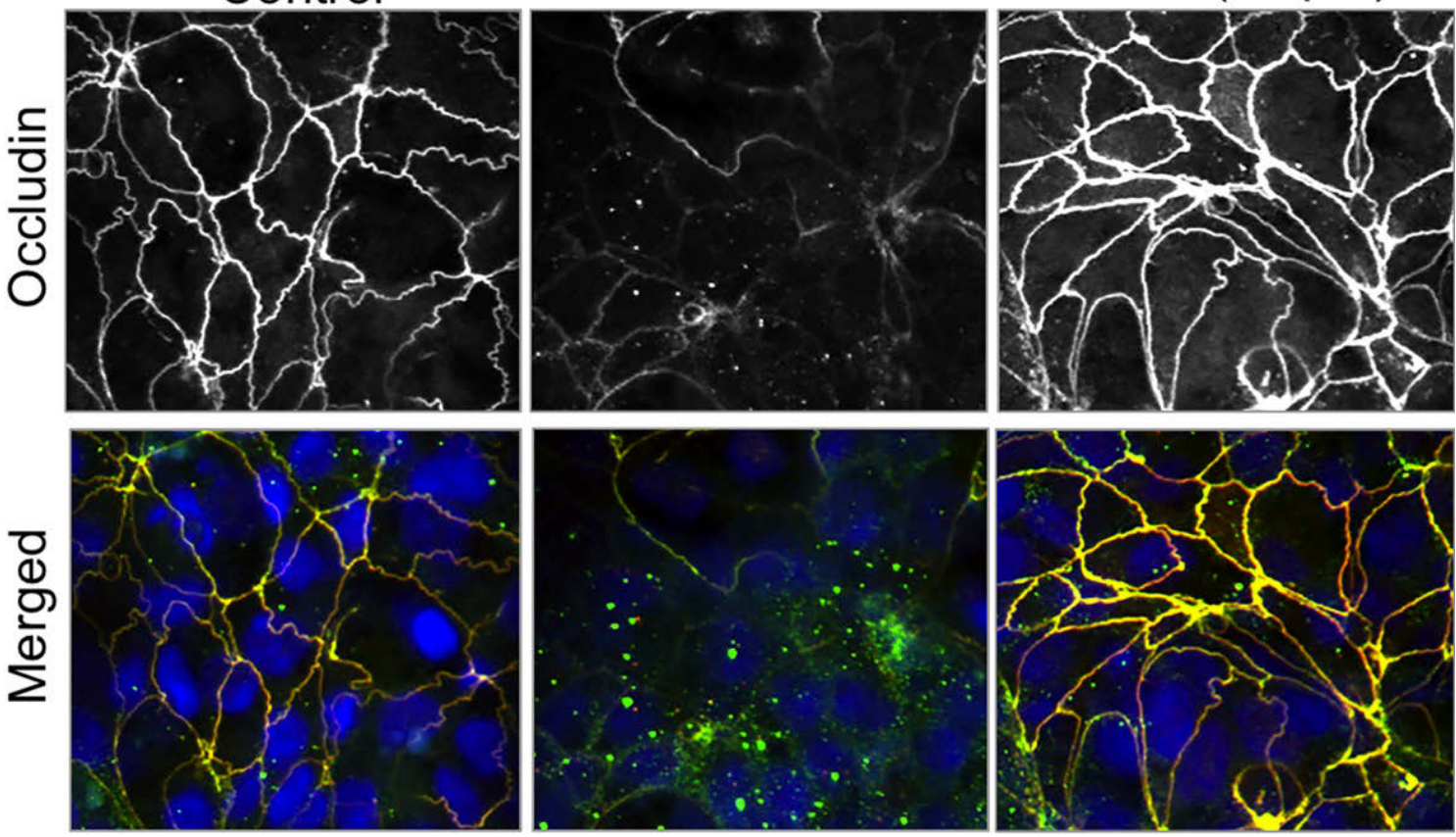

Green - Occludin; Red - ZO-1; Blue - Nucleus

Figure 3-22. ORP Attenuates Radiation-induced TJ Disruption.

Cell monolayers pretreated with $10 \mu \mathrm{M}$ ORP were exposed to radiation (4Gy) and fixed at $2 \mathrm{hr}$ post-radiation. Monolayers were immunostained for occludin (Green), ZO-1 (Red) and nucleus (Blue). 
using streptavidin agarose. The beads were isolated and subjected to LC-MS/MS analysis. By a bottom-up proteomics approach, the proteins pulled down in each sample were identified. Immunoblot analysis was performed for selective proteins.

The proteins identified in each group were statistically analyzed and proteins were shortlisted based on the statistical scores (Table 3-1). Some proteins were pulled down by peptide irrespective of its phosphorylation status, like Peroxiredoxin 1 (PRDX1), where as some proteins pulled down were found exclusively in each group, like microtubule associated protein (MAP7). These data suggested that proteins interact with ORM in a phosphorylation-dependent mechanism. The ion spectra profiles of HSPD1, PRDX1, MAP7 and PKP3 peptides are provided in the figure (Figure 3-23).

Immunoblot analyses was performed for PRDX1, Heat shock protein 60 (HSPD1), Plakophilin 3 (PKP3) and MAP7. This confirmed interaction of native peptide with HSP 60 and PRDX1. On the other hand, immunoblot for MAP7 and plakophilin 3 showed an enhanced interaction between tyrosine phosphorylated ORP and MAP7 as well as plakophilin3 (Figure 3-24). These data confirmed that ORP binds to proteins in a phosphorylation-dependent manner.

\section{MAP7 Binds to Tyrosine-Phosphorylated ORP and GST-Ocl-C}

MAP7 is an epithelial specific microtubule associated protein known to be an essential cofactor for Kinesin-1 driven transport. Microtubule is believed to mediate actomyosin contraction and internalization of TJ proteins form junctions during $\mathrm{Ca}^{2+}$ depletion mediated TJ disruption. In order to validate the interaction between ORM and MAP7, both ORP and recombinant GST-Ocl-C were used. GST-Ocl-C comprises of the distal 150 amino acids of the occludin C-terminus. ORP and GST-Ocl-C were prephosphorylated with c-Src in presence or absence of ATP and used as baits for the pull down assay using either streptavidin agarose or GSH agarose beads. ORPscr and GST were used as their respective controls. The protein complexes were immunoblotted for MAP7.

MAP7 was pulled down in case of ORP (NP) but not ORPscr. There was a significant increase in the amount of MAP7 pulled with ORP-pY, indicating that tyrosine phosphorylation enhances interaction between ORM and MAP7 (Figure 3-25A). Similarly, MAP7 was pulled down exclusively by GST-Ocl-C-pY (Figure 3-25B), suggesting that MAP7 interacts with tyrosine phosphorylated occludin C-terminus.

\section{DSS or Osmotic Stress Enhances Interaction Between Occludin and MAP7}

In order to validate the interaction between full length occludin and MAP7, coimmunoprecipitation analysis was performed in Caco-2 cells. Previous studies showed that both DSS and osmotic stress induce tyrosine phosphorylation of occludin $(83,84)$. 
Table 3-1. ORM-interacting Proteins Identified by LC-MS/MS.

\begin{tabular}{|c|c|c|}
\hline $\begin{array}{l}\text { ORP (NP) } \\
\text { (Total Proteins-78) }\end{array}$ & $\begin{array}{l}\text { ORP (pY) } \\
\text { (Total Proteins-91) }\end{array}$ & $\begin{array}{l}\text { ORP }(\mathrm{pS} / \mathrm{T}) \\
\text { (Total Proteins-85) }\end{array}$ \\
\hline Peroxiredoxin-1 (PRDX1) & Ensconsin (MAP7) & THO complex subunit 4 \\
\hline Acetyl CoA acetyltransferase & Plakophilin-3 (PKP3) & Phosphate carrier protein \\
\hline Protein disulfide-isomerase A6 & Peroxiredoxin 1 (PRDX1) & RNA helicase \\
\hline Heat shock protein (HSPD1) & RNA helicase & Peroxiredoxin-1 \\
\hline Protein disisomerase A6 & Tubulin beta- $4 \mathrm{~A}$ & RNA binding protein EWS \\
\hline ATP-citrate synthase & Elongation factor $1 \mathrm{a}$ & Glucose regulated protein \\
\hline Triose phosphate isomerase & POU-domain binding protein & Glutathione peroxidase-1 \\
\hline Glucose regulated protein & Heat shock protein (HSPD1) & Tubulin beta-4a \\
\hline Fatty acid synthase & Glutathione-S-transferase & Thiamine transporter 1 \\
\hline Thiamine transporter 1 & Glutathione peroxidase & Heat shock protein (HSPD1) \\
\hline
\end{tabular}

Note: The table here enlists the statistically significant proteins $(\mathrm{p} \leq 0.05)$ identified in pull down assay using non-phosphorylated ORP, tyrosine phosphorylated ORP (ORP-pY) and serine/threonine phosphorylated ORP (ORP-pS/T). 



Figure 3-23. Ion Spectra Profiles of Peptides Corresponding to the Proteins Identified by LC-MS/MS.

Represented here are the ion sprectra profiles of peptides corresponding to peroxiredoxin 1(PRDX1), heat shock protein 60 (HSPD1), microtubule associated protein (MAP7) and plakophilin 3 (PKP3). 
A



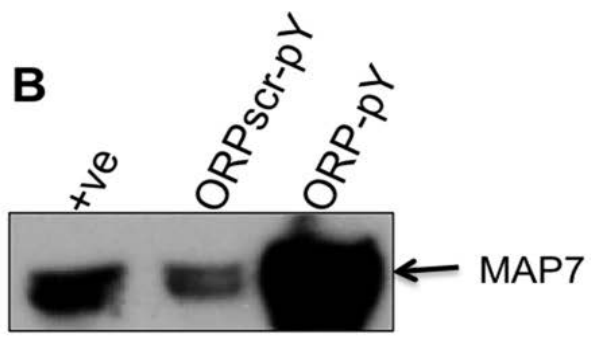

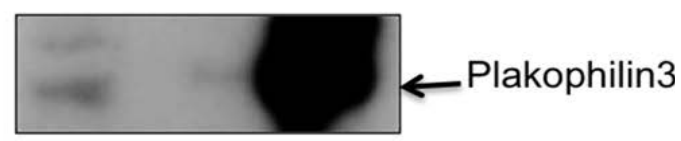

Figure 3-24. Immunoblot Analyses for Proteins Pulled Down by ORP.

A: ORP or ORPscr in their native form were incubated with native cell extracts and subjected to pull down using streptavidin agarose. Protein complexes were immunoblotted for HSP60 and Peroxiredoxin1. B: Peptides were pre-phosphorylated with active c-Src (ORP-pY or ORPscr) and incubated with native cell extracts for the pull down. Protein complexes were immunoblotted for MAP7 and Plakophilin3.

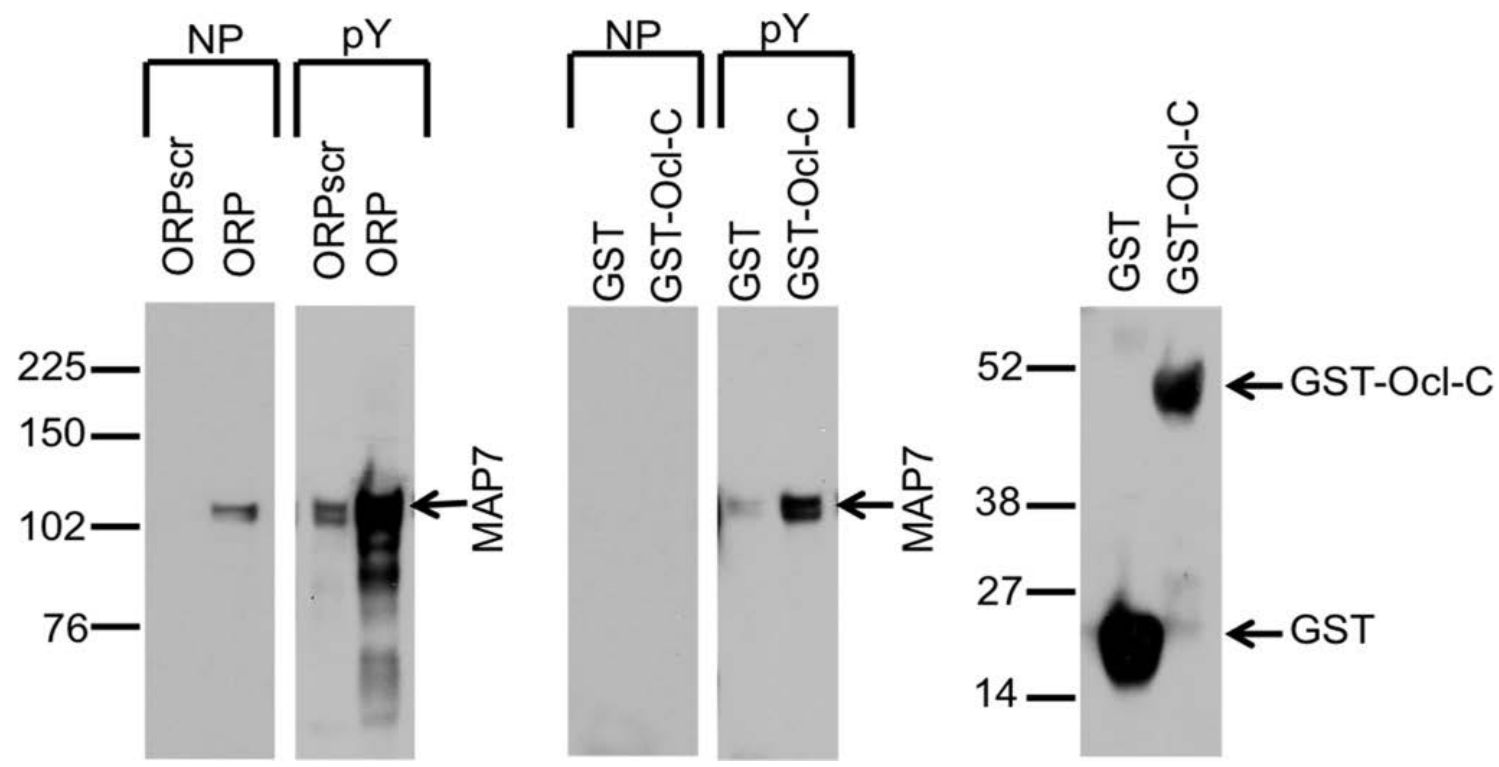

Figure 3-25. MAP7 Interacts with Tyrosine-phosphorylated ORP and Occludin Cterminus.

A \& B: ORP and ORPscr (A) or GST and GST-Ocl-C (B) in their native form or prephosphorylated with active-Src were incubated with Caco-2 cell extracts. Protein complexes were immunoblotted for MAP7 and GST. 
Caco-2 cells were treated with either 3\% DSS or osmotic stress. MAP7 was

immunoprecipitated from the native cell extracts and immunoblotted for occludin and MAP7.

The immunoblot showed a minimal interaction between occludin and MAP7 in controls. However, this interaction was significantly enhanced by both DSS and osmotic stress (Figure 3-26A). The densitometric analysis confirmed that tyrosine phosphorylation enhanced interaction between occludin and MAP7 (Figure 3-26B). This suggested that MAP7 interacts with occludin in a tyrosine phosphorylation dependent mechanism.

\section{MAP7 Colocalizes with Occludin in Irradiated Mouse Colon}

To validate the interaction between MAP7 and occludin in vivo, C57BL/6 mice were subjected to radiation (4Gy) and intestinal tissues were harvested after 2 hours. Cryosections of distal colon were fixed and immunostained for occludin and MAP7. High-resolution images were collected by confocal microscopy.

In the sham treated mouse colon, MAP7 had an intracellular distribution located near the perijunctional region. No colocalization of occludin and MAP7 was seen in the absence of radiation. However, MAP7 appeared to colocalize with occludin in irradiated mouse colon (Figure 3-27). These data suggested that radiation-induced tyrosine phosphorylation of occludin results in a potential interaction between occludin and MAP7. 

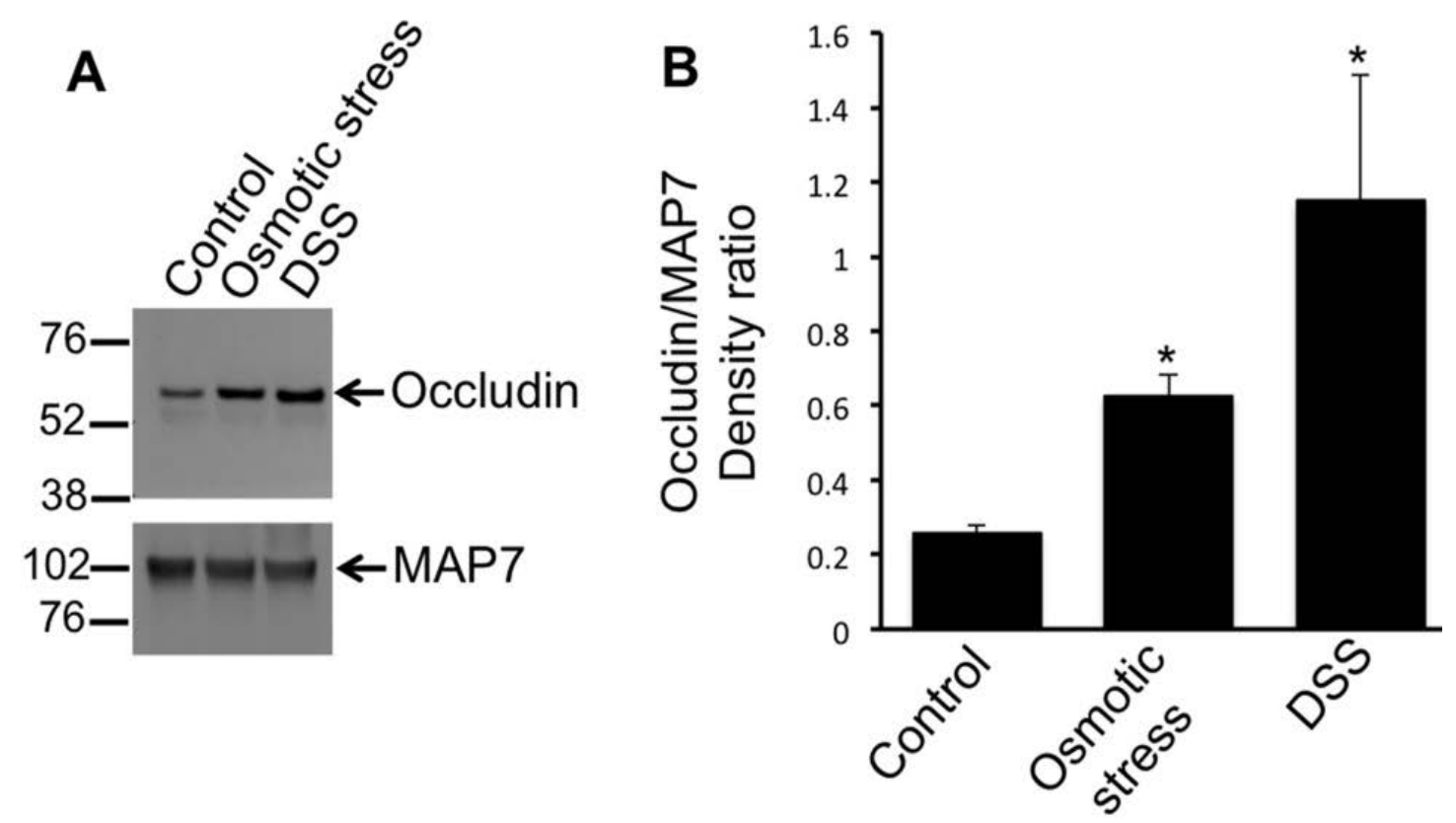

Figure 3-26. MAP7 Interacts with Occludin in a Tyrosine phosphorylationdependent Mechanism.

A: MAP7 was immunoprecipitated from cell extracts isolated from Caco- 2 cells treated with either $0.35 \mathrm{M}$ mannitol or 3\% DSS for $3 \mathrm{hr}$. Immune-complexes were blotted for MAP7 and occludin. B: Density of occludin was measured and normalized to corresponding MAP7 band density. Values presented in the graph are mean \pm seem $(\mathrm{n}=$ $3)$. Asterisk indicates that the value is significantly $(\mathrm{p}<0.05)$ different from corresponding control value. 

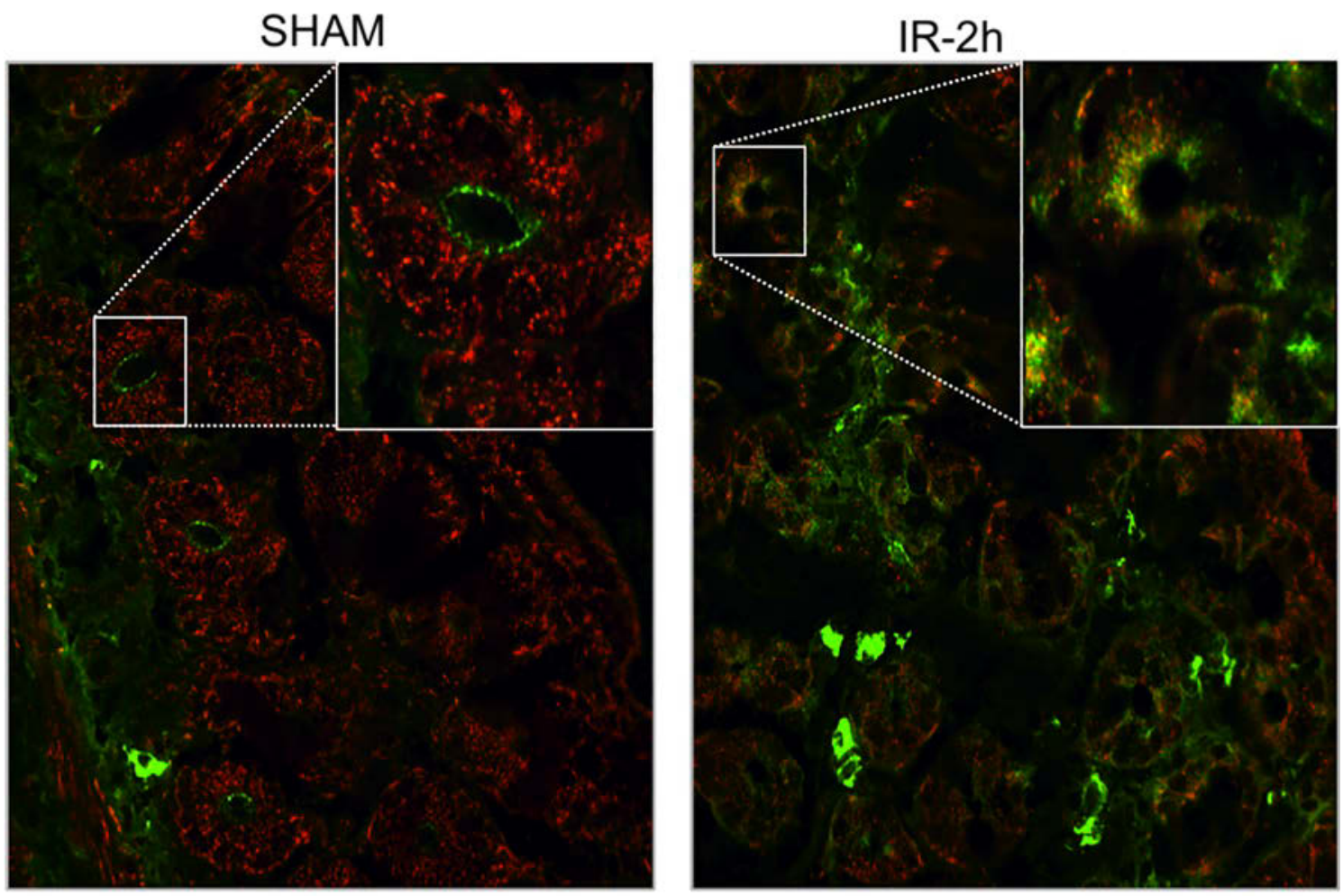

Green: Occludin; Red: MAP7

Figure 3-27. MAP7 Colocalizes with Occludin in Radiation Exposed Mouse Colon.

Mice (C57BL/6) were subjected to radiation (4Gy) and intestinal tissues were harvested 2 hr. post-radiation. Cryosections of distal colon were fixed and labeled for occludin (Green) and MAP7 (Red). Images were collected by confocal microscopy. Inset shows zoomed-in regions of colocalization. 


\section{CHAPTER 4. DISCUSSION}

\section{Specific Aim 1: To Determine That ORM Confers Dynamics to TJ in a Phosphorylation-dependent Mechanism}

Occludin is considered as a functional component of TJ. The phosphorylation hot spot in occludin (ORM) is a potential regulatory platform that mediates phosphorylationdependent regulation of TJ. Several disorders have been shown to involve occludin redistribution from $\mathrm{TJ}$ with the associated increase in permeability and barrier dysfunction. Hence determining the function of ORM is crucial to understand TJ modulation both in the aspects of its physiology as well as disease pathogenesis.

\section{ORM Is Not Required for Assembly of Occludin into TJ}

Previous studies demonstrated that loss of occludin does not prevent TJ assembly, in both in vitro and in vivo models $(35,36)$. The intracellular transport of occludin is governed by its C-terminus (37). C-terminal sequence of $\sim 150$ amino acids (358-504) was believed to be required for occludin localization at TJ (14). However, Bald et al showed that C-terminally truncated chicken occludin localized at TJ in MDCK cells, although with a discontinuous distribution (85). A study by Chen et al using Xenopus embryos showed that C-terminally truncated mutant chicken occludin localized at TJ by its ability to oligomerize with endogenous occudin (38). In order to study the function of ORM, we first generated deletion mutant of occludin $\left(\mathrm{Ocl}^{\mathrm{DM}}\right)$ that lacks ORM. To avoid potential interactions with the endogenous occludin, we transfected $\mathrm{Ocl}^{\mathrm{DM}}$ into occludin deficient-MDCK cells and generated stable clones. As ORM is located in the C-terminus we examined whether the deletion of ORM affected the assembly of occludin into TJ.

Consistent with the previous data, lack of ORM did not affect assembly of $\mathrm{Ocl}^{\mathrm{DM}}$ into TJ, indicating that ORM is not required for TJ assembly.

The association of C-terminal sequence (358-504) with ZO-1 is required for occludin assembly at TJ (18). Structural analysis revealed that the distal C-terminal region (416-522) of occludin forms a coiled-coil structure that was sufficient for interaction with the guanylate kinase domain (GUK) of ZO-1 (31). Because ORM is located in close proximity with the coiled coil domain, we investigated if the interaction between occludin and ZO-1 was affected by deletion of ORM. Our studies showed that ZO-1 co-immunoprecipitated with GFP-Ocl ${ }^{\mathrm{DM}}$ similar to GFP-Ocl $^{\mathrm{WT}}$. This indicates that ORM is not required for the interaction between occludin and ZO-1. Based on these observations we concluded that ORM is not required for occludin assembly into intercellular junctions. 


\section{ORM Deletion Does Not Affect Barrier Development by Leak Pathway Flux}

Expression of both wild type and $\mathrm{COOH}$-terminally truncated chicken occludin in MDCK cells induced an increase in TER, but only the truncated occludin expression induced an increase in paracellular permeability (40). Another study demonstrated that knock down of occludin in MDCK cells altered paracellular ion permeability but not steady-state TER (86). To determine the effect of ORM deletion on barrier function, post seeding barrier development was assessed. Similar to $\mathrm{Ocl}^{\mathrm{WT}}$, expression of $\mathrm{Ocl}^{\mathrm{DM}}$ showed a decrease in inulin permeability by day 4 , indicating that the absence of ORM does not affect the barrier development. Interestingly, even on day 4 the resistance of $\mathrm{Ocl}^{\mathrm{DM}}$ and Vec cell monolayers remained lower than that in $\mathrm{Ocl}^{\mathrm{DM}}$ cell monolayers, although the inulin permeability was not different. This observation suggested that the pore pathway might be affected by ORM deletion, while leak pathway remains unaffected.

TER is a measure of the flux of all ions across the epithelium. Small ions do not discriminate between pore and leak pathways. A change in TER with unaltered leak pathway permeability implicated a change in pore pathway permeability. The expression of a subset of claudins that have the ability to form pores is known to determine paracellular permeability (87). In order to account for the differences in TER in these clones, we examined the expression and distribution of claudin-2, a major cationic pore forming claudin (25). A previous study has shown that S408 phosphorylation of occludin regulates claudin-2 localization in Caco-2 cell monolayers (27). Immunoblot analysis and confocal microscopy showed that claudin-2 levels and its junctional distribution were greater in $\mathrm{Ocl}^{\mathrm{DM}}$ and $\mathrm{Vec}$ cell monolayers compared to that of $\mathrm{Ocl}^{\mathrm{WT}}$ cell monolayers. Results indicated that the enhanced junctional localization of claudin-2 might have contributed to lower TER in these cell lines. These data suggest that barrier development in terms of TJ assembly at intercellular junctions is not affected by ORM deletion, however TJ regulation-mediated flux through pore pathway is affected in the absence of ORM. We showed for the first time that ORM might be involved in the regulation of pore pathway.

\section{ORM Deletion Enhances Occludin Localization and Reduces Its Mobility at the TJ}

The actin cytoskeleton is a dynamic network that maintains cellular architecture and tissue organization. Transmission electron microscopy and immunogold labeling studies revealed that a perijunctional actomyosin ring is located below the apical junctional complex $(88,89)$. The cytoplasmic TJ plaque is closely associated with the cortical cytoskeleton via interactions with the scaffolding protein, ZO-1 (12). Early studies using pharmacological agents of disruption also revealed that the cytoskeleton plays an important role in regulating TJ permeability (88). It was implicated that cytoskeletal organization is important for maintenance of the TJ architecture and hence their sealing properties. Occludin C-terminus is known to interact with the actomyosin ring indirectly via its interaction with ZO-1(90). Here we examined the cytoskeletal organization of cells lacking ORM or occludin, during barrier development and the distribution of occludin between detergent soluble and detergent insoluble fractions. 
Confocal microscopy showed that actin cytoskeleton was better organized at the intercellular junctions of $\mathrm{Ocl}^{\mathrm{DM}}$ and Vec cell monolayers compared to $\mathrm{Ocl}^{\mathrm{WT}}$ cell monolayers. Immunoblot analysis suggested that lack of ORM enhanced association of occudin with the actin rich triton insoluble fraction.

Studies by Turner group have established the fact that TJ undergoes continuous remodeling at steady state. FRAP based studies by Shen et al (6) demonstrated that TJ proteins are highly dynamic and that they are in constant exchange with extra-junction pools. It was believed that it is through this plasticity of constant exchange with extrajunction pools, TJ proteins respond to external and internal stimuli involved in paracellular barrier regulation. Each TJ protein has a distinct dynamic behavior. They showed that occludin was the most mobile among all the TJ proteins and exchanges by diffusion within and between TJ and lateral membrane. From our studies, FRAP analysis of the dynamic behavior of occludin showed that deletion of ORM makes occludin at the intercellular junctions, more static and less mobile. ORM may be required for this mobility of occludin. We believe that the increased association of $\mathrm{Ocl}^{\mathrm{DM}}$ with the actin cytoskeleton contributes to the reduced mobility.

\section{ORM Deletion Specifically Attenuates $\mathrm{Ca}^{2+}$ Depletion-mediated Disruption of Apical Junctional Complexes and Barrier Dysfunction}

$\mathrm{Ca}^{2+}$ switch assay is a widely used technique to study de novo assembly of TJ, however the mechanism involved in $\mathrm{Ca}^{2+}$ depletion-mediated $\mathrm{TJ}$ disassembly or $\mathrm{Ca}^{2+}$ repletion-mediated TJ resassembly has not been identified. However, it is believed that the extracellular $\mathrm{Ca}^{2+}$ depletion-induced AJ disruption, as E-cadherin requires $\mathrm{Ca}^{2+}$ for its hemophilic interactions, leads to disruption of TJ. Another probable mechanism likely involved is that the depletion of extracellular $\mathrm{Ca}^{2+}$ leads to a decrease in intracellular $\mathrm{Ca}^{2+}$, which activates a signaling cascade that disassembles TJ and AJ components. ORM deletion attenuated $\mathrm{Ca}^{2+}$ depletion-induced TJ, AJ, cytoskeleton disruption and barrier function.

The lack of an effect of LCM on junctional distribution of GFP-Ocl ${ }^{\text {DM }}$ suggested that ORM is required for $\mathrm{Ca}^{2+}$ depletion -mediated redistribution of occludin. The lack of an effect of LCM on junctional distribution of $\mathrm{ZO}-1$ in $\mathrm{Ocl}^{\mathrm{DM}}$ cell monolayers indicates that absence of ORM prevents disruption of $\mathrm{TJ}$ by $\mathrm{Ca}^{2+}$ depletion. Sustained junctional organization of E-cadherin and b-catenin in LCM-treated $\mathrm{Ocl}^{\mathrm{DM}}$ cell monolayers suggests that ORM deletion prevents disruption of $\mathrm{AJ}$ by $\mathrm{Ca}^{2+}$ depletion. Stability of actin cytoskeleton and microtubules in LCM treated $\mathrm{Ocl}^{\mathrm{DM}}$ cell monolayers demonstrates that lack of ORM blocks $\mathrm{Ca}^{2+}$ depletion -mediated reorganization of actin cytoskeleton and microtubules. Changes in TER and inulin flux by LCM and $\mathrm{Ca}^{2+}$ restoration indicates that barrier function is disrupted by $\mathrm{Ca}^{2+}$ depletion and restored by $\mathrm{Ca}^{2+}$ repletion in $\mathrm{Ocl}^{\mathrm{WT}}$ cell monolayers. Absence of such changes in TER and inulin-flux in $\mathrm{Ocl}^{\mathrm{DM}}$ and $\mathrm{Vec}$ monolayers, suggests that ORM is required for $\mathrm{Ca}^{2+}$ depletion mediated barrier dysfunction in MDCK cell monolayers. Similar results in experiments using EGTA mediated $\mathrm{Ca}^{2+}$ depletion model further confirmed the role of ORM in TJ and AJ 
disruption. Thus ORM plays a likely role in mediating TJ assembly/disassembly during $\mathrm{Ca}^{2+}$ switch assay.

Cytochalasin D is a well-known disruptor of the actin-cytoskeleton and disruption of actin cytoskeleton has been shown to disrupt epithelial TJ (91). Cytochalasin-D mediated decrease in TER, increase in inulin-flux and redistribution of occludin in both $\mathrm{Ocl}^{\mathrm{WT}}$ and $\mathrm{Ocl}^{\mathrm{DM}}$ cell monolayers suggests that ORM deletion does not affect cytochalasin D-mediated TJ disruption and barrier dysfunction. Similarly, data show that ORM deletion does not affect osmotic stress-mediated TJ disruption and barrier dysfunction. Mechanical disruption by cytochalasin-D and osmotic stress might have caused a drastic effect and irreversible damage to the TJ and hence the effect of ORM deletion on barrier function was not evident in these cases.

\section{Phosphorylation of ORM on T403/T404 and Y398/Y402 Determines the Dynamic Properties of TJ}

Previous studies in MDCK cells showed that expression of occludin with T403/404A mutation attenuated TJ assembly, while that with T403/404D mutation enhanced TJ assembly after $\mathrm{Ca}^{2+}$ switch (35). The results here show that T403/404A, but not T403/404D, mutation confers resistance to $\mathrm{Ca}^{2+}$ depletion-mediated redistribution of occludin and ZO-1 and barrier dysfunction, similar to ORM deletion mutation. This suggested that T403 and T404 phosphorylation of occludin is required for mediating $\mathrm{Ca}^{2+}$ depletion-mediated TJ disruption. Also T403/404A, but not T403/404D mutation reduces occludin mobility at the intercellular junctions similar to ORM deletion mutation. This suggested that T403 and T404 phosphorylation contributes to mobility of occludin at the intercellular junctions. In other words, the dynamic properties contributed by T403 and T404 phosphorylation enables internalization of occudin during TJ disassembly while its absence reduces mobile fraction of occludin and resists $\mathrm{TJ}$ disassembly by $\mathrm{Ca}^{2+}$ depletion.

Previous studies showed that expression of occludin with Y398/402A mutation did not alter occludin localization at TJ during reassembly while that with Y398/402D mutation delayed $\mathrm{TJ}$ assembly after $\mathrm{Ca}^{2+}$ switch. From the current data, resistance to LCM-mediated barrier dysfunction and occludin/ZO-1 redistribution in the presence of genistein confirmed that tyrosine phosphorylation is involved in $\mathrm{Ca}^{2+}$ depletion-mediated TJ disruption. Y398/402A and Y398/402D mutations confer partial resistance to $\mathrm{Ca}^{2+}$ depletion-mediated redistribution of occludin/ZO-1 and TJ disruption, indicating that both tyrosine phosphorylation and dephosphorylation may be involved in $\mathrm{Ca}^{2+}$ depletionmediated TJ disruption. The decrease in mobility due to both Y398/402A and Y398/402D mutations suggested that tyrosine phosphorylation/dephosphorylation also confer dynamics to TJ. These data together demonstrate that ORM confers dynamics to TJ by a phosphorylation-dependent mechanism. 


\section{Specific Aim 2: To Determine That ORM-mediated Protein Interactions Are Involved in TJ Modulation}

We believed that the translation of occludin phosphorylation signal into TJ modulation, is mediated by proteins that interact with ORM. In order to further establish the significance of ORM phosphorylation in TJ modulation, the strategy employed was aimed to design a peptide that mimics ORM and investigate ORM-mediated protein interactions.

\section{ORP Enhances TJ Assembly and Barrier Function}

Crystallography studies on occludin C-terminus determined that the distal part of this domain (416-522) forms a coiled-coil structure but the proximal region from 383-415 does assume a secondary structure (31). Our data (unpublished) from studies on chicken occludin C-terminus using hydrogen deuterium exchange coupled mass spectrometry (HDE-MS) showed that there was significantly higher incorporation of deuterium in the peptide corresponding to ORM sequence. On the other hand, the residues Y398, Y402, T403, T404 and S408 are present as a cluster in a sequence, which is highly conserved among species $(34,92)$. From this information, it was evident that ORM is an active site of phosphorylation that is readily available for ORM-mediated protein interactions.

To identify the proteins that interact with ORM, a peptide analogous to ORM sequence (ORP) was designed with an N-terminus biotin tag and C-terminus TAT domain. Examination of ORP permeability in Caco2-cells and mouse colonic loops, showed increased uptake of ORP by the cells compared to that of the scrambled control. The idea was to have ORP mimicking endogenous ORM sequence. Then the biological effect of ORP was tested. Treatment with ORP enhanced TJ assembly and barrier function during $\mathrm{Ca}^{2+}$ switch. Balda et al, showed that Occludin C-terminus governs intracellular transport of occludin (85). This showed that the intracellular ORP might have enabled occludin transport to the TJ and enhanced barrier function.

To further validate the biological effect of barrier function, a radiation model was used. Caco- 2 cells when exposed to radiation showed a decrease in TER and increase in inulin-flux. However, when cells were pretreated with ORP, the radiation-induced decrease in TER, increase in flux and redistribution of occludin and ZO-1 was significantly attenuated. ORP was thought to attenuate radiation-induced barrier dysfunction by enhancing ORM-mediated protein interactions involved in maintaining TJ integrity. Thus far, the studies revealed that ORP has a potential to mimic endogenous ORM and positively regulate $\mathrm{TJ}$.

\section{ORP Interacts with Proteins in a Phosphorylation-dependent Manner}

In order to identify the proteins that interact with ORM, ORP was used as bait in pull down assay coupled-mass spectrometry. ORM is differentially phosphorylated under 
different physiological conditions. Tyrosine phosphorylation of occludin is associated with TJ disruption while threonine phosphorylation is involved in TJ assembly (36). To identify proteins that interact with differentially phosphorylated ORM, ORP was prephosphorylated in vitro. Thus the method to phosphorylate in vitro was validated in our previous studies. The same principle was employed to selectively phosphorylate ORP either by incubation with c-Src, which is a tyrosine kinase, or CK2, which is a serine, threonine kinase. Three forms of ORP were used as baits in the pull down assay: ORP in its native form, tyrosine phosphorylated ORP (ORP-pY) and serine/threonine phosphorylated ORP (ORP-pS/T).

Mass spectrometry of the pulled down samples was able to identify several proteins in each group. The protein hits were further analyzed using Scaffold software and statistically significant proteins were shortlisted. Some proteins were pulled down in all three groups while some were unique to each group indicating the phosphorylation dependent binding of proteins. I chose four proteins for further validation by immunoblot analyses. PRDX1 and HSPD1 were pulled down in all the three groups while MAP7 and PKP3 were pulled down exclusively by ORP-pY.

PRDX1 is a protein with dual roles since it acts as a peroxidase and as a chaperone (93). PRDX1 is upregulated in human colorectal cancer and breast cancer $(94,95)$, both the cases where occludin downregulation was reported $(69,96)$. Although not much is known about role of PRDX1 in TJ, based on our pull down results it was speculated that PRDX1 interaction with ORM could have a potential role in chaperoning TJ proteins like occludin under physiological and pathophysiological conditions. PRDX1 however was pulled down with ORP irrespective of its phosphorylation status. Another protein MAP7, which is a microtubule associated protein, was pulled down only by tyrosine phosphorylated ORP. Since tyrosine phosphorylation is associated with TJ disruption, an interaction between ORM and MAP7 indicated the existence of a potential mechanism mediating occludin trafficking during stress. Hence we focused on investigating the significance of the interaction between MAP7 and ORM.

\section{MAP7 Interacts with Occludin by a Phosphorylation-dependent Mechanism}

Because ORP is a synthetic peptide, it was essential to establish the interaction of MAP7 with occludin in its native form. First a recombinant occludin C-terminus tagged to GST was used to perform a pull down assay similar to that with ORP. Immunoblot analysis for MAP7 and GST revealed that MAP7 bound specifically to GST-Ocl-C-pY. This suggested that MAP7 directly interacts with occludin C-terminus, mediated by tyrosine phosphorylation.

To further validate this interaction between full length occludin and MAP7, coimmunoprecipitation analyses was carried out in Caco-2 cells. Since MAP7 binds to tyrosine-phosphorylated ORM, a stimulus that induced occludin phosphorylation was needed. Osmotic stress and DSS are known to induce JNK and c-Src mediated phosphorylation of occludin $(83,84)$. Caco-2 cells were treated with either osmotic stress 
or DSS and MAP7 was immunoprecipitated from the native extracts. Immunoblot analysis showed that although there was a basal amount of interaction between MAP7 and occludin, this was significantly enhanced by treatment with both osmotic stress and DSS. This suggested that tyrosine phosphorylation of occludin enhances interaction with MAP7 possibly mediated by the activation of JNK and c-Src.

Then I investigated the interaction between occludin and MAP7 in vivo. Radiation causes TJ remodeling by inducing oxidative stress (97). Occludin undergoes tyrosine phosphorylation during oxidative stress-induced disruption of TJ $(52,53,98)$. Hence a radiation model was used to induce tyrosine phosphorylation of occludin in vivo. Mice were subjected to radiation and intestinal tissues were harvested $2 \mathrm{hr}$ post radiation. Colonic cryosections immunostained for occludin and MAP7 showed marked differences between radiation treated and sham treated mice. Sites of occludin, MAP7 colocalization were observed in the radiation exposed mouse colon but not in the sham treated control. This suggested that MAP7 interacts with occludin which is tyrosine phosphorylated possibly by the oxidative stress induced by ionizing radiation.

Ivanov et al., demonstrated that TJ disassembly is regulated by microtubules (99). TJ disruption involves microtubule-mediated contraction of actomyosin ring as well as microtubule-mediated internalization of TJ proteins. It was shown that Kinesin-1 is a motor protein that drives dynamic reorganization of microtubules and trafficking of TJ proteins. Kinesin-1 colocalizes with occludin at TJ as well as with occludin that was internalized by $\mathrm{Ca}^{2+}$ depletion (99). MAP7 is an epithelial specific microtubule associated protein and an essential cofactor for kinesin-1 $(100,101)$. These observations suggested that MAP7 is the potential regulatory protein that in association with Kinesin-1 drives the trafficking of tyrosine-phosphorylated occludin during TJ disruption (Figure 4-1).

\section{Summary}

In conclusion, this series of studies determined the functional significance of ORM in TJ regulation. These studies identified that ORM plays an important role in mediating TJ assembly/disassembly during $\mathrm{Ca}^{2+}$ switch assay. The correlation between occludin phosphorylation and TJ modulation is further supported by the observation that ORM-mediated phosphorylation confers dynamic properties to TJ. They also shed light on the potential role of ORM in regulating pore pathway flux, which is mediated by poreforming claudins. MAP7, for the first time, has been identified as an ORM-interacting partner that mediates trafficking of occludin during TJ disruption. ORM is a highly dynamic site that recruits signaling molecules to modulate $\mathrm{TJ}$, a mechanism most likely exploited during pathogenesis of several diseases associated with barrier dysfunction. The data here unravels novel mechanisms and associated proteins involved in TJ regulation that might prove to be potential therapeutic targets for $\mathrm{TJ}$ modulation. 


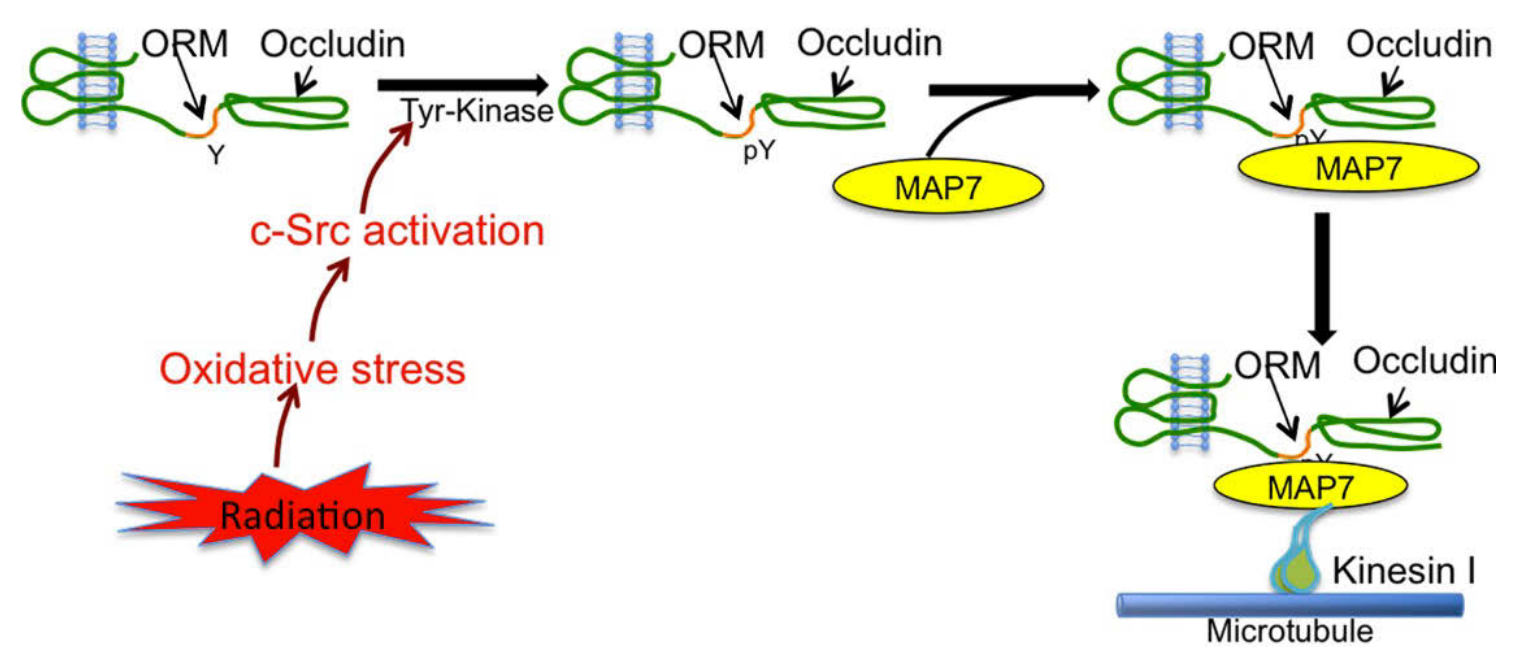

Figure 4-1. MAP7 Mediates Trafficking of Occludin During TJ Regulation.

ORM is a site for phosphorylation dependent regulation of occludin. Tyrosine phosphorylation of occludin induces interaction with MAP7, which in association with kinesin-1 mediates dynamic regulation of occludin. Under pathological conditions such as radiation-induced oxidative stress, tyrosine kinase like c-Src is activated. Sustained phosphorylation of tyrosine residues in ORM enhances interaction with MAP7, which in association with kinesin-1 and microtubule mediates internalization of occludin leading to TJ modulation. 


\section{LIST OF REFERENCES}

1. Farquhar, M. G., and Palade, G. E. (1963) Junctional complexes in various epithelia. J Cell Biol 17, 375-412

2. Tsukita, S., Furuse, M., and Itoh, M. (2001) Multifunctional strands in tight junctions. Nat Rev Mol Cell Biol 2, 285-293

3. Shen, L., Weber, C. R., and Turner, J. R. (2008) The tight junction protein complex undergoes rapid and continuous molecular remodeling at steady state. $J$ Cell Biol 181, 683-695

4. Wang, F., Graham, W. V., Wang, Y., Witkowski, E. D., Schwarz, B. T., and Turner, J. R. (2005) Interferon-gamma and tumor necrosis factor-alpha synergize to induce intestinal epithelial barrier dysfunction by up-regulating myosin light chain kinase expression. The American journal of pathology 166, 409-419

5. Madara, J. L., and Stafford, J. (1989) Interferon-gamma directly affects barrier function of cultured intestinal epithelial monolayers. The Journal of clinical investigation 83, 724-727

6. Hecht, G., Pothoulakis, C., LaMont, J. T., and Madara, J. L. (1988) Clostridium difficile toxin A perturbs cytoskeletal structure and tight junction permeability of cultured human intestinal epithelial monolayers. The Journal of clinical investigation 82, 1516-1524

7. Yuhan, R., Koutsouris, A., Savkovic, S. D., and Hecht, G. (1997) Enteropathogenic Escherichia coli-induced myosin light chain phosphorylation alters intestinal epithelial permeability. Gastroenterology 113, 1873-1882

8. Groschwitz, K. R., and Hogan, S. P. (2009) Intestinal barrier function: molecular regulation and disease pathogenesis. J Allergy Clin Immunol 124, 3-20; quiz 2122

9. Elamin, E., Jonkers, D., Juuti-Uusitalo, K., van Ijzendoorn, S., Troost, F., Duimel, H., Broers, J., Verheyen, F., Dekker, J., and Masclee, A. (2012) Effects of ethanol and acetaldehyde on tight junction integrity: in vitro study in a three dimensional intestinal epithelial cell culture model. PLoS One 7, e35008

10. Schneeberger, E. E., and Lynch, R. D. (1992) Structure, function, and regulation of cellular tight junctions. The American journal of physiology 262, L647-661

11. Gumbiner, B. (1990) Generation and maintenance of epithelial cell polarity. Current opinion in cell biology 2, 881-887

12. Anderson, J. M., and Van Itallie, C. M. (1995) Tight junctions and the molecular basis for regulation of paracellular permeability. The American journal of physiology 269, G467-475

13. Matter, K., and Balda, M. S. (1999) Occludin and the functions of tight junctions. Int Rev Cytol 186, 117-146

14. Matter, K., and Balda, M. S. (2003) Signalling to and from tight junctions. Nat Rev Mol Cell Biol 4, 225-236

15. Stevenson, B. R., Siliciano, J. D., Mooseker, M. S., and Goodenough, D. A. (1986) Identification of ZO-1: a high molecular weight polypeptide associated with the tight junction (zonula occludens) in a variety of epithelia. J Cell Biol 103, 755-766 
16. Citi, S., Sabanay, H., Jakes, R., Geiger, B., and Kendrick-Jones, J. (1988)

Cingulin, a new peripheral component of tight junctions. Nature 333, 272-276

17. Furuse, M., Hirase, T., Itoh, M., Nagafuchi, A., Yonemura, S., Tsukita, S., and Tsukita, S. (1993) Occludin: a novel integral membrane protein localizing at tight junctions. J Cell Biol 123, 1777-1788

18. Furuse, M., Itoh, M., Hirase, T., Nagafuchi, A., Yonemura, S., Tsukita, S., and Tsukita, S. (1994) Direct association of occludin with ZO-1 and its possible involvement in the localization of occludin at tight junctions. J Cell Biol 127, 1617-1626

19. Van Itallie, C. M., and Anderson, J. M. (1997) Occludin confers adhesiveness when expressed in fibroblasts. Journal of cell science 110 ( Pt 9), 1113-1121

20. Furuse, M., Fujita, K., Hiiragi, T., Fujimoto, K., and Tsukita, S. (1998) Claudin-1 and -2: novel integral membrane proteins localizing at tight junctions with no sequence similarity to occludin. J Cell Biol 141, 1539-1550

21. Anderson, J. M., and Van Itallie, C. M. (2009) Physiology and function of the tight junction. Cold Spring Harb Perspect Biol 1, a002584

22. Raleigh, D. R., Marchiando, A. M., Zhang, Y., Shen, L., Sasaki, H., Wang, Y., Long, M., and Turner, J. R. (2010) Tight junction-associated MARVEL proteins marveld3, tricellulin, and occludin have distinct but overlapping functions.

Molecular biology of the cell 21, 1200-1213

23. Dorfel, M. J., Westphal, J. K., and Huber, O. (2009) Differential phosphorylation of occludin and tricellulin by CK2 and CK1. Annals of the New York Academy of Sciences 1165, 69-73

24. Gunzel, D., and Yu, A. S. (2013) Claudins and the modulation of tight junction permeability. Physiol Rev 93, 525-569

25. Amasheh, S., Meiri, N., Gitter, A. H., Schoneberg, T., Mankertz, J., Schulzke, J. D., and Fromm, M. (2002) Claudin-2 expression induces cation-selective channels in tight junctions of epithelial cells. Journal of cell science 115, 49694976

26. Muto, S., Hata, M., Taniguchi, J., Tsuruoka, S., Moriwaki, K., Saitou, M., Furuse, K., Sasaki, H., Fujimura, A., Imai, M., Kusano, E., Tsukita, S., and Furuse, M. (2010) Claudin-2-deficient mice are defective in the leaky and cation-selective paracellular permeability properties of renal proximal tubules. Proc Natl Acad Sci $U S A$ 107, 8011-8016

27. Shin, K., Fogg, V. C., and Margolis, B. (2006) Tight junctions and cell polarity. Annual review of cell and developmental biology 22, 207-235

28. Feldman, G. J., Mullin, J. M., and Ryan, M. P. (2005) Occludin: structure, function and regulation. Adv Drug Deliv Rev 57, 883-917

29. Cummins, P. M. (2012) Occludin: one protein, many forms. Mol Cell Biol 32, 242-250

30. Dorfel, M. J., and Huber, O. (2012) Modulation of tight junction structure and function by kinases and phosphatases targeting occludin. J Biomed Biotechnol 2012, 807356

31. Li, Y., Fanning, A. S., Anderson, J. M., and Lavie, A. (2005) Structure of the conserved cytoplasmic C-terminal domain of occludin: identification of the ZO-1 binding surface. J Mol Biol 352, 151-164 
32. Rao, R. K., Baker, R. D., Baker, S. S., Gupta, A., and Holycross, M. (1997) Oxidant-induced disruption of intestinal epithelial barrier function: role of protein tyrosine phosphorylation. The American journal of physiology 273, G812-823

33. Kale, G., Naren, A. P., Sheth, P., and Rao, R. K. (2003) Tyrosine phosphorylation of occludin attenuates its interactions with ZO-1, ZO-2, and ZO-3. Biochem Biophys Res Commun 302, 324-329

34. Elias, B. C., Suzuki, T., Seth, A., Giorgianni, F., Kale, G., Shen, L., Turner, J. R., Naren, A., Desiderio, D. M., and Rao, R. (2009) Phosphorylation of Tyr-398 and Tyr-402 in occludin prevents its interaction with ZO-1 and destabilizes its assembly at the tight junctions. J Biol Chem 284, 1559-1569

35. Suzuki, T., Elias, B. C., Seth, A., Shen, L., Turner, J. R., Giorgianni, F., Desiderio, D., Guntaka, R., and Rao, R. (2009) PKC eta regulates occludin phosphorylation and epithelial tight junction integrity. Proc Natl Acad Sci U S A 106, 61-66

36. Rao, R. (2009) Occludin phosphorylation in regulation of epithelial tight junctions. Annals of the New York Academy of Sciences 1165, 62-68

37. Raleigh, D. R., Boe, D. M., Yu, D., Weber, C. R., Marchiando, A. M., Bradford, E. M., Wang, Y., Wu, L., Schneeberger, E. E., Shen, L., and Turner, J. R. (2011) Occludin S408 phosphorylation regulates tight junction protein interactions and barrier function. $J$ Cell Biol 193, 565-582

38. Dorfel, M. J., Westphal, J. K., Bellmann, C., Krug, S. M., Cording, J., Mittag, S., Tauber, R., Fromm, M., Blasig, I. E., and Huber, O. (2013) CK2-dependent phosphorylation of occludin regulates the interaction with $\mathrm{ZO}$-proteins and tight junction integrity. Cell Commun Signal 11, 40

39. Furuse, M., Fujimoto, K., Sato, N., Hirase, T., Tsukita, S., and Tsukita, S. (1996) Overexpression of occludin, a tight junction-associated integral membrane protein, induces the formation of intracellular multilamellar bodies bearing tight junction-like structures. Journal of cell science 109 ( Pt 2), 429-435

40. Balda, M. S., Whitney, J. A., Flores, C., Gonzalez, S., Cereijido, M., and Matter, K. (1996) Functional dissociation of paracellular permeability and transepithelial electrical resistance and disruption of the apical-basolateral intramembrane diffusion barrier by expression of a mutant tight junction membrane protein. $J$ Cell Biol 134, 1031-1049

41. Saitou, M., Fujimoto, K., Doi, Y., Itoh, M., Fujimoto, T., Furuse, M., Takano, H., Noda, T., and Tsukita, S. (1998) Occludin-deficient embryonic stem cells can differentiate into polarized epithelial cells bearing tight junctions. J Cell Biol 141, 397-408

42. Saitou, M., Furuse, M., Sasaki, H., Schulzke, J. D., Fromm, M., Takano, H., Noda, T., and Tsukita, S. (2000) Complex phenotype of mice lacking occludin, a component of tight junction strands. Molecular biology of the cell 11, 4131-4142

43. Beeman, N. E., Baumgartner, H. K., Webb, P. G., Schaack, J. B., and Neville, M. C. (2009) Disruption of occludin function in polarized epithelial cells activates the extrinsic pathway of apoptosis leading to cell extrusion without loss of transepithelial resistance. BMC cell biology 10, 85

44. Rachow, S., Zorn-Kruppa, M., Ohnemus, U., Kirschner, N., Vidal-y-Sy, S., von den Driesch, P., Bornchen, C., Eberle, J., Mildner, M., Vettorazzi, E., Rosenthal, 
R., Moll, I., and Brandner, J. M. (2013) Occludin is involved in adhesion, apoptosis, differentiation and $\mathrm{Ca} 2+$-homeostasis of human keratinocytes: implications for tumorigenesis. PLoS One 8, e55116

45. Van Itallie, C. M., Fanning, A. S., Holmes, J., and Anderson, J. M. (2010) Occludin is required for cytokine-induced regulation of tight junction barriers. Journal of cell science 123, 2844-2852

46. McKenzie, J. A., Riento, K., and Ridley, A. J. (2006) Casein kinase I epsilon associates with and phosphorylates the tight junction protein occludin. FEBS letters 580, 2388-2394

47. Cordenonsi, M., Turco, F., D'Atri, F., Hammar, E., Martinucci, G., Meggio, F., and Citi, S. (1999) Xenopus laevis occludin. Identification of in vitro phosphorylation sites by protein kinase CK2 and association with cingulin. European journal of biochemistry / FEBS 264, 374-384

48. Chen, Y. H., Lu, Q., Goodenough, D. A., and Jeansonne, B. (2002) Nonreceptor tyrosine kinase c-Yes interacts with occludin during tight junction formation in canine kidney epithelial cells. Molecular biology of the cell 13, 1227-1237

49. Seth, A., Sheth, P., Elias, B. C., and Rao, R. (2007) Protein phosphatases 2A and 1 interact with occludin and negatively regulate the assembly of tight junctions in the CACO-2 cell monolayer. $J$ Biol Chem 282, 11487-11498

50. Nunbhakdi-Craig, V., Machleidt, T., Ogris, E., Bellotto, D., White, C. L., 3rd, and Sontag, E. (2002) Protein phosphatase 2A associates with and regulates atypical $\mathrm{PKC}$ and the epithelial tight junction complex. J Cell Biol 158, 967-978

51. Sallee, J. L., and Burridge, K. (2009) Density-enhanced phosphatase 1 regulates phosphorylation of tight junction proteins and enhances barrier function of epithelial cells. J Biol Chem 284, 14997-15006

52. Sheth, P., Basuroy, S., Li, C., Naren, A. P., and Rao, R. K. (2003) Role of phosphatidylinositol 3-kinase in oxidative stress-induced disruption of tight junctions. J Biol Chem 278, 49239-49245

53. Basuroy, S., Sheth, P., Kuppuswamy, D., Balasubramanian, S., Ray, R. M., and Rao, R. K. (2003) Expression of kinase-inactive c-Src delays oxidative stressinduced disassembly and accelerates calcium-mediated reassembly of tight junctions in the Caco-2 cell monolayer. J Biol Chem 278, 11916-11924

54. Andreeva, A. Y., Piontek, J., Blasig, I. E., and Utepbergenov, D. I. (2006) Assembly of tight junction is regulated by the antagonism of conventional and novel protein kinase $\mathrm{C}$ isoforms. The international journal of biochemistry \& cell biology 38, 222-233

55. Nusrat, A., Chen, J. A., Foley, C. S., Liang, T. W., Tom, J., Cromwell, M., Quan, C., and Mrsny, R. J. (2000) The coiled-coil domain of occludin can act to organize structural and functional elements of the epithelial tight junction. $J$ Biol Chem 275, 29816-29822

56. Rao, R. K., Basuroy, S., Rao, V. U., Karnaky Jr, K. J., and Gupta, A. (2002) Tyrosine phosphorylation and dissociation of occludin-ZO-1 and E-cadherin-betacatenin complexes from the cytoskeleton by oxidative stress. The Biochemical journal 368, 471-481 
57. Jain, S., Suzuki, T., Seth, A., Samak, G., and Rao, R. (2011) Protein kinase Czeta phosphorylates occludin and promotes assembly of epithelial tight junctions. The Biochemical journal 437, 289-299

58. Sheth, P., Samak, G., Shull, J. A., Seth, A., and Rao, R. (2009) Protein phosphatase 2 A plays a role in hydrogen peroxide-induced disruption of tight junctions in Caco-2 cell monolayers. The Biochemical journal 421, 59-70

59. Dunagan, M., Chaudhry, K., Samak, G., and Rao, R. K. (2012) Acetaldehyde disrupts tight junctions in Caco-2 cell monolayers by a protein phosphatase 2Adependent mechanism. American journal of physiology. Gastrointestinal and liver physiology 303, G1356-1364

60. Mankertz, J., Tavalali, S., Schmitz, H., Mankertz, A., Riecken, E. O., Fromm, M., and Schulzke, J. D. (2000) Expression from the human occludin promoter is affected by tumor necrosis factor alpha and interferon gamma. Journal of cell science 113 ( Pt 11), 2085-2090

61. Liu, X., Dreffs, A., Diaz-Coranguez, M., Runkle, E. A., Gardner, T. W., Chiodo, V. A., Hauswirth, W. W., and Antonetti, D. A. (2016) Occludin S490

Phosphorylation Regulates Vascular Endothelial Growth Factor-Induced Retinal Neovascularization. The American journal of pathology 186, 2486-2499

62. Saitou, M., Ando-Akatsuka, Y., Itoh, M., Furuse, M., Inazawa, J., Fujimoto, K., and Tsukita, S. (1997) Mammalian occludin in epithelial cells: its expression and subcellular distribution. Eur J Cell Biol 73, 222-231

63. Muresan, Z., Paul, D. L., and Goodenough, D. A. (2000) Occludin 1B, a variant of the tight junction protein occludin. Molecular biology of the cell 11, 627-634

64. Drago, S., El Asmar, R., Di Pierro, M., Grazia Clemente, M., Tripathi, A., Sapone, A., Thakar, M., Iacono, G., Carroccio, A., D'Agate, C., Not, T., Zampini, L., Catassi, C., and Fasano, A. (2006) Gliadin, zonulin and gut permeability: Effects on celiac and non-celiac intestinal mucosa and intestinal cell lines. Scandinavian journal of gastroenterology 41, 408-419

65. McNamara, B. P., Koutsouris, A., O'Connell, C. B., Nougayrede, J. P., Donnenberg, M. S., and Hecht, G. (2001) Translocated EspF protein from enteropathogenic Escherichia coli disrupts host intestinal barrier function. The Journal of clinical investigation 107, 621-629

66. Singh, U., Van Itallie, C. M., Mitic, L. L., Anderson, J. M., and McClane, B. A. (2000) CaCo-2 cells treated with Clostridium perfringens enterotoxin form multiple large complex species, one of which contains the tight junction protein occludin. J Biol Chem 275, 18407-18417

67. Wu, Z., Nybom, P., and Magnusson, K. E. (2000) Distinct effects of Vibrio cholerae haemagglutinin/protease on the structure and localization of the tight junction-associated proteins occludin and ZO-1. Cellular microbiology 2, 11-17

68. Nusrat, A., von Eichel-Streiber, C., Turner, J. R., Verkade, P., Madara, J. L., and Parkos, C. A. (2001) Clostridium difficile toxins disrupt epithelial barrier function by altering membrane microdomain localization of tight junction proteins.

Infection and immunity 69, 1329-1336

69. Wang, X., Tully, O., Ngo, B., Zitin, M., and Mullin, J. M. (2011) Epithelial tight junctional changes in colorectal cancer tissues. ScientificWorldJournal 11, 826841 
70. Sawada, N. (2013) Tight junction-related human diseases. Pathology international 63, 1-12

71. Tobioka, H., Isomura, H., Kokai, Y., Tokunaga, Y., Yamaguchi, J., and Sawada, N. (2004) Occludin expression decreases with the progression of human endometrial carcinoma. Hum Pathol 35, 159-164

72. Rao, R. (2009) Endotoxemia and gut barrier dysfunction in alcoholic liver disease. Hepatology (Baltimore, Md.) 50, 638-644

73. Mir, H., Meena, A. S., Chaudhry, K. K., Shukla, P. K., Gangwar, R., Manda, B., Padala, M. K., Shen, L., Turner, J. R., Dietrich, P., Dragatsis, I., and Rao, R. (2016) Occludin deficiency promotes ethanol-induced disruption of colonic epithelial junctions, gut barrier dysfunction and liver damage in mice. Biochim Biophys Acta 1860, 765-774

74. Ploss, A., Evans, M. J., Gaysinskaya, V. A., Panis, M., You, H., de Jong, Y. P., and Rice, C. M. (2009) Human occludin is a hepatitis C virus entry factor required for infection of mouse cells. Nature 457, 882-886

75. Benedicto, I., Molina-Jimenez, F., Bartosch, B., Cosset, F. L., Lavillette, D., Prieto, J., Moreno-Otero, R., Valenzuela-Fernandez, A., Aldabe, R., LopezCabrera, M., and Majano, P. L. (2009) The tight junction-associated protein occludin is required for a postbinding step in hepatitis $\mathrm{C}$ virus entry and infection. J Virol 83, 8012-8020

76. Wang, Z., Mandell, K. J., Parkos, C. A., Mrsny, R. J., and Nusrat, A. (2005) The second loop of occludin is required for suppression of Rafl-induced tumor growth. Oncogene 24, 4412-4420

77. Li, D., and Mrsny, R. J. (2000) Oncogenic Raf-1 disrupts epithelial tight junctions via downregulation of occludin. J Cell Biol 148, 791-800

78. Chen, Y., Lu, Q., Schneeberger, E. E., and Goodenough, D. A. (2000) Restoration of tight junction structure and barrier function by down-regulation of the mitogenactivated protein kinase pathway in ras-transformed Madin-Darby canine kidney cells. Molecular biology of the cell 11, 849-862

79. Yamamoto, M., Ramirez, S. H., Sato, S., Kiyota, T., Cerny, R. L., Kaibuchi, K., Persidsky, Y., and Ikezu, T. (2008) Phosphorylation of claudin-5 and occludin by rho kinase in brain endothelial cells. The American journal of pathology 172, 521533

80. Papadopoulos, M. C., Saadoun, S., Woodrow, C. J., Davies, D. C., Costa-Martins, P., Moss, R. F., Krishna, S., and Bell, B. A. (2001) Occludin expression in microvessels of neoplastic and non-neoplastic human brain. Neuropathology and applied neurobiology 27, 384-395

81. Morgan, L., Shah, B., Rivers, L. E., Barden, L., Groom, A. J., Chung, R., Higazi, D., Desmond, H., Smith, T., and Staddon, J. M. (2007) Inflammation and dephosphorylation of the tight junction protein occludin in an experimental model of multiple sclerosis. Neuroscience 147, 664-673

82. Chen, Y., Merzdorf, C., Paul, D. L., and Goodenough, D. A. (1997) COOH terminus of occludin is required for tight junction barrier function in early Xenopus embryos. J Cell Biol 138, 891-899

83. Samak, G., Chaudhry, K. K., Gangwar, R., Narayanan, D., Jaggar, J. H., and Rao, R. (2015) Calcium/Ask1/MKK7/JNK2/c-Src signalling cascade mediates 
disruption of intestinal epithelial tight junctions by dextran sulfate sodium. The Biochemical journal 465, 503-515

84. Samak, G., Narayanan, D., Jaggar, J. H., and Rao, R. (2011) CaV1.3 channels and intracellular calcium mediate osmotic stress-induced N-terminal c-Jun kinase activation and disruption of tight junctions in Caco-2 CELL MONOLAYERS. $J$ Biol Chem 286, 30232-30243

85. Matter, K., and Balda, M. S. (1998) Biogenesis of tight junctions: the C-terminal domain of occludin mediates basolateral targeting. Journal of cell science 111 ( Pt 4), 511-519

86. Yu, A. S., McCarthy, K. M., Francis, S. A., McCormack, J. M., Lai, J., Rogers, R. A., Lynch, R. D., and Schneeberger, E. E. (2005) Knockdown of occludin expression leads to diverse phenotypic alterations in epithelial cells. Am J Physiol Cell Physiol 288, C1231-1241

87. Kirk, A., Campbell, S., Bass, P., Mason, J., and Collins, J. (2010) Differential expression of claudin tight junction proteins in the human cortical nephron. Nephrol Dial Transplant 25, 2107-2119

88. Madara, J. L., Stafford, J., Barenberg, D., and Carlson, S. (1988) Functional coupling of tight junctions and microfilaments in T84 monolayers. The American journal of physiology 254, G416-423

89. Drenckhahn, D., and Dermietzel, R. (1988) Organization of the actin filament cytoskeleton in the intestinal brush border: a quantitative and qualitative immunoelectron microscope study. J Cell Biol 107, 1037-1048

90. Fanning, A. S., Jameson, B. J., Jesaitis, L. A., and Anderson, J. M. (1998) The tight junction protein $\mathrm{ZO}-1$ establishes a link between the transmembrane protein occludin and the actin cytoskeleton. J Biol Chem 273, 29745-29753

91. Madara, J. L., Barenberg, D., and Carlson, S. (1986) Effects of cytochalasin D on occluding junctions of intestinal absorptive cells: further evidence that the cytoskeleton may influence paracellular permeability and junctional charge selectivity. J Cell Biol 102, 2125-2136

92. Dorfel, M. J., and Huber, O. (2012) A phosphorylation hotspot within the occludin C-terminal domain. Annals of the New York Academy of Sciences 1257, 38-44

93. Saccoccia, F., Di Micco, P., Boumis, G., Brunori, M., Koutris, I., Miele, A. E., Morea, V., Sriratana, P., Williams, D. L., Bellelli, A., and Angelucci, F. (2012) Moonlighting by different stressors: crystal structure of the chaperone species of a 2-Cys peroxiredoxin. Structure 20, 429-439

94. Rho, J. H., Qin, S., Wang, J. Y., and Roehrl, M. H. (2008) Proteomic expression analysis of surgical human colorectal cancer tissues: up-regulation of PSB7, PRDX1, and SRP9 and hypoxic adaptation in cancer. J Proteome Res 7, 29592972

95. Cha, M. K., Suh, K. H., and Kim, I. H. (2009) Overexpression of peroxiredoxin I and thioredoxin1 in human breast carcinoma. $J$ Exp Clin Cancer Res 28, 93

96. Martin. (2010) Loss of occludin leads to the progression of human breast cancer. International Journal of Molecular Medicine 26

97. Shukla, P. K., Gangwar, R., Manda, B., Meena, A. S., Yadav, N., Szabo, E., Balogh, A., Lee, S. C., Tigyi, G., and Rao, R. (2016) Rapid disruption of 
intestinal epithelial tight junction and barrier dysfunction by ionizing radiation in mouse colon in vivo: protection by N-acetyl-1-cysteine. American journal of physiology. Gastrointestinal and liver physiology 310, G705-715

98. Rao, R. (2008) Oxidative stress-induced disruption of epithelial and endothelial tight junctions. Front Biosci 13, 7210-7226

99. Ivanov, A. I., McCall, I. C., Babbin, B., Samarin, S. N., Nusrat, A., and Parkos, C. A. (2006) Microtubules regulate disassembly of epithelial apical junctions. $B M C$ cell biology 7, 12

100. Barlan, K., Lu, W., and Gelfand, V. I. (2013) The microtubule-binding protein ensconsin is an essential cofactor of kinesin-1. Current biology : $C B$ 23, 317-322

101. Gallaud, E., Caous, R., Pascal, A., Bazile, F., Gagne, J. P., Huet, S., Poirier, G. G., Chretien, D., Richard-Parpaillon, L., and Giet, R. (2014) Ensconsin/Map7 promotes microtubule growth and centrosome separation in Drosophila neural stem cells. J Cell Biol 204, 1111-1121 


\section{VITA}

Bhargavi Manda was born as Bhargavi Jetti in Andhra Pradesh, India in 1985. She received a Bachelor of Science degree from St.Ann's college, Hyderabad in 2004 and a Master of Science in Biochemistry from Bhavan's Vivekananda College, Hyderabad in 2009. She joined the Integrated Biomedical Sciences Program at the University of Tennessee Health Science Center in August 2011. She joined the lab of Dr. Radhakrishna Rao in January 2012. During her Ph.D. in Dr. RK Rao's lab, she presented her work at national conferences in Boston and Washington DC. She is a co-author for nearly 8 publications and is going to submit two first author manuscripts. She will be obtaining a Doctor of Philosophy degree in Biomedical sciences with concentration in cell biology and physiology before leaving UTHSC. With the knowledge and expertise she gained during her Ph.D. at UTHSC, she is looking forward to working in the field of Translational Medicine. 